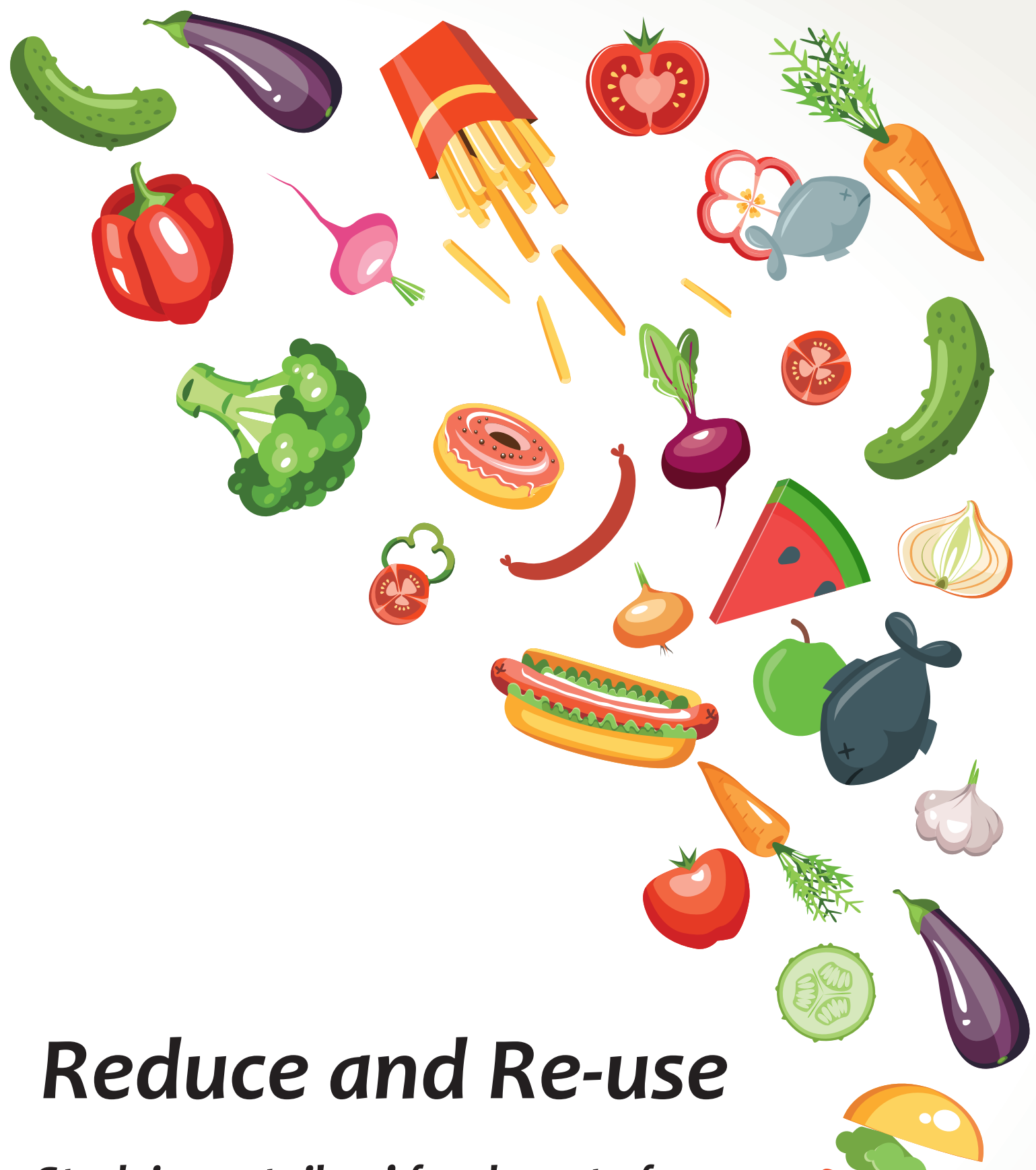

Studying retailers' food waste from an Operations Research perspective 


\section{Propositions}

1. Food waste reduction should be seen as a multi-objective decision problem. (this thesis)

2. To reduce food waste, consumer choices must be limited. (this thesis)

3. To obtain feasible solutions for problems involving humanity, improper modelling of human behaviour is necessary.

4. Full time jobs increase individualism.

(Dekker, R., et al. (2008). Economisch Statistische Berichten, 93, 404-407.)

5. The greatest excitement occurs when there is nothing to see.

6. Research is a hobby, except for PhD students.

Propositions belonging to the thesis, entitled

Reduce and Re-use: Studying retailers' food waste from an Operations Research perspective

Marjolein Elize Buisman

Wageningen, 19 September 2019 


\section{Reduce and re-use: \\ Studying retailers' food waste from an Operations Research perspective}




\section{Thesis committee}

\section{Promotor}

Prof. dr Jaqueline M. Bloemhof

Professor of Operations Research and Logistics

Wageningen University \& Research

\section{Co-promotor}

Dr Rene Haijema

Associate Professor, Operations Research and Logistics

Wageningen University \& Research

\section{Other members}

Prof. dr Jacques H. Trienekens, Wageningen University \& Research

Prof. dr Iris F.A. Vis, University of Groningen

Prof. dr Alexander Hübner, Technical University München, Germany

Dr Sander L.J.M. de Leeuw, VU University, Amsterdam

This research was conducted under the auspices of the Wageningen School of Social Sciences (WASS) 


\title{
Reduce and re-use: \\ Studying retailers' food waste from an Operations Research perspective
}

\author{
Marjolein Elize Buisman
}

Thesis

submitted in fulfilment of the requirements for the degree of doctor at

Wageningen University

by the authority of the Rector Magnificus

Prof. dr A.P.J. Mol,

in the presence of the

Thesis Committee appointed by the Academic Board

to be defended in public

on Thursday 19 September 2019

at 11.00 a.m. in the Aula. 
Marjolein Elize Buisman

Reduce and re-use: Studying retailers' food waste from an Operations Research perspective 152 pages.

PhD thesis, Wageningen University, Wageningen, The Netherlands (2019)

With references, with summary in English

ISBN 978-94-6395-041-1

DOI https://doi.org/10.18174/496523 


\section{Contents}

Page

Contents $\quad$ v

Chapter 1 Introduction $\quad 1$

1.1 Global food waste . . . . . . . . . . . . . . . . . . 2

1.2 Food waste by retailers . . . . . . . . . . . . . . . . . 3

1.3 Possible interventions to reduce food waste at the retailer . . . . . . . . . 5

1.4 Research outline and aims . . . . . . . . . . . . . . . . . . 7

Chapter 2 Discounting and dynamic shelf life to reduce fresh food waste $\begin{array}{ll}\text { at retailers } & 11\end{array}$

2.1 Introduction . . . . . . . . . . . . . . . . . . . . . . 13

2.2 Literature . . . . . . . . . . . . . . . . . . . . . . . . 14

2.3 Method . . . . . . . . . . . . . . . . . . 17

2.4 Numerical results . . . . . . . . . . . . . . . . . . . . . . . . 24

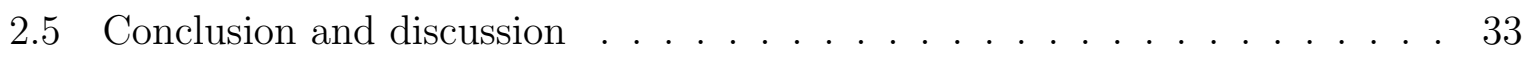

Chapter 3 Product substitution to increase profit and reduce food waste $\begin{array}{ll}\text { at retailers } & 37\end{array}$

3.1 Introduction . . . . . . . . . . . . . . . . . . . . . . . . . . . . 39

3.2 Related literature . . . . . . . . . . . . . . . . . . . . . . . . . . . . . . . . . . . .

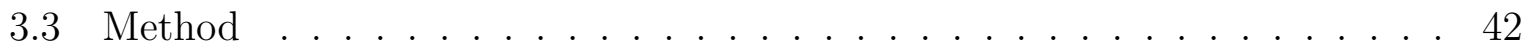

3.4 Heuristic to find optimal order-up-to levels with one-way substitution . . . 48

3.5 Numerical results . . . . . . . . . . . . . . . . . . . . . . . . . . . . . 49

3.6 Conclusion and discussion $\ldots \ldots \ldots \ldots \ldots$. . . . . . . . . . . . . . 57

Chapter 4 Inventory optimization under consumer driven substitution for vertically differentiated products $\quad 59$

4.1 Introduction . . . . . . . . . . . . . . . . . . . . . . 61

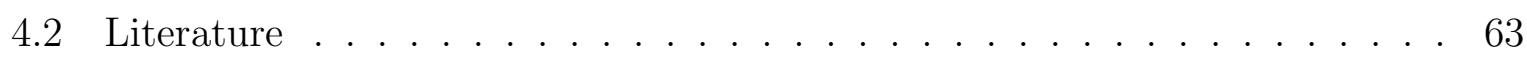

4.3 Model formulation . . . . . . . . . . . . . . . . . . 66 
4.4 Solution procedure . . . . . . . . . . . . . . . . . . . 72

4.5 Numerical results . . . . . . . . . . . . . . . . . . . . . . . . . . . . . . . . . . . . . . . . . . . . . . . . .

4.6 Conclusion and discussion . . . . . . . . . . . . . . . . . . . 81

Chapter 5 Donation management for menu planning at soup kitchens $\quad 85$

5.1 Introduction . . . . . . . . . . . . . . . . . 87

5.2 Related literature . . . . . . . . . . . . . . . . . . . . . . . . . . . . . . . . . . . . . . . .

5.3 Modelling approach . . . . . . . . . . . . . . . . . . . 91

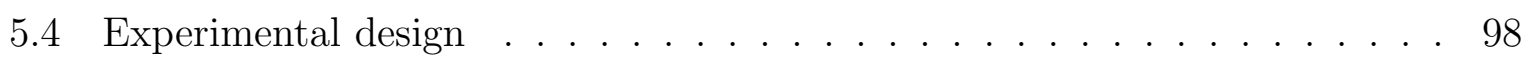

5.5 Numerical results . . . . . . . . . . . . . . . . . . . . . . . . 100

5.6 Decision rules for accepting ad hoc donations . . . . . . . . . . . . . 107

5.7 Conclusion and discussion . . . . . . . . . . . . . . . . . . . 111

Chapter 6 Conclusions and general discussion $\quad 115$

6.1 Conclusions . . . . . . . . . . . . . . . . . . . 116

6.2 Integrated findings and scientific contribution . . . . . . . . . . . . 119

6.3 Managerial discussion . . . . . . . . . . . . . . . . . . . . . 122

6.4 Further research . . . . . . . . . . . . . . . . 125

$\begin{array}{lr}\text { References } & 129\end{array}$

$\begin{array}{ll}\text { Summary } & 143\end{array}$

$\begin{array}{ll}\text { Publications } & 145\end{array}$

$\begin{array}{ll}\text { Acknowledgements } & 147\end{array}$

$\begin{array}{ll}\text { Completed Training and Supervision Plan } & 149\end{array}$ 
Chapter 1

\section{Introduction}




\subsection{Global food waste}

In the $21^{\text {st }}$ century, the food system faces major environmental and social challenges due to the continuous growth the demand for food worldwide (Godfray et al., 2010). By 2040, the global food consumption is expected to grow by $70-100 \%$ (World Bank, 2007). To cope with this growth, a shift towards a more sustainable food system is needed, including a more efficient use of resources such as land and water, an increase in production yield and a reduction of food loss (Thyberg \& Tonjes, 2016).

Global food losses are estimated to represent around $50 \%$ of the total worldwide production, or one third of the edible food produced (Lundqvist et al., 2008; Gustavsson et al., 2011). For the European Union (EU), food waste is estimated at 100 million tons per year, or $173 \mathrm{~kg}$ per capita (Stenmarck et al., 2016). Food waste or food loss occurs at all stages of the food supply chain, with more than two thirds happening in the final stages of the supply chain (see Figure 1.1). To address food waste within the EU, the EU Horizon2020 project REFRESH was launched. This project aims to reduce and re-use the food and beverage wasted within the EU, with a holistic approach for the entire supply chain (Monier et al., 2010). The work described in this thesis is carried out within this European project, in collaboration with WRAP, Wageningen Food \& Biobased Research and many other partners. More information on this project can be found at www.eu-refresh.org.

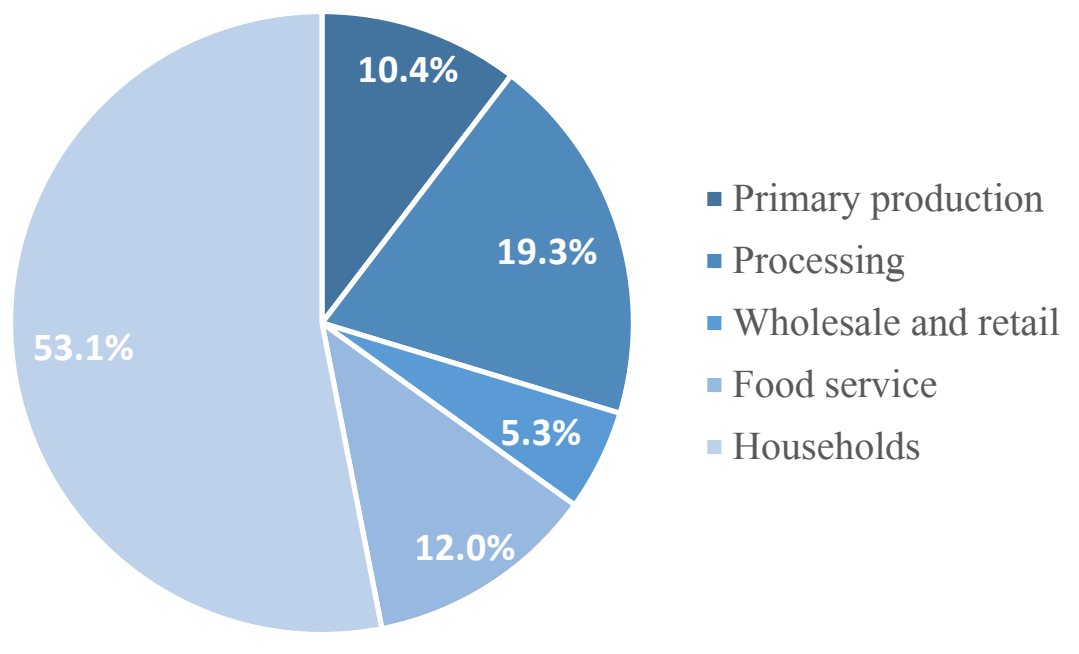

Figure 1.1: Percentage of food waste at different stages of the EU food supply chain (Stenmarck et al., 2016)

In general, a distinction is made between food loss and food waste; food loss is the decrease in edible food mass, which would otherwise be categorised as suitable for human 
consumption at later stages of production, whereas food waste is considered to be the discarded products that have been produced for human consumption (Thyberg \& Tonjes, 2016). Thus, food waste tends to occur in later the stages of the supply chain, such as retail and consumption, while food losses usually occur at the beginning of the food supply chain, including post-harvest or during processing. In addition to the classification of food waste and food loss, one can also distinguish between avoidable and unavoidable food waste. Bones and peels are considered unavoidable food waste, as they are not suitable for human consumption; however, some of these items could still be re-used for other purposes (Stenmarck et al., 2011). The share of unavoidable food waste differs for the various stages of the supply chain. At the consumer the fraction of unavoidable food waste is between 20-50\%, while for the manufacturer (food processing step), the unavoidable food waste is estimated at $50 \%$ of the total waste (Van Westerhoven, 2013; Harris, 2017). At the retailer however, all food waste is considered avoidable, as everything is intended for sale (Parfitt et al., 2016). In this thesis, the focus is on avoidable food waste; thus we will not consider food loss and unavoidable food waste.

The global food production is currently sufficient to feed the world's population; however, due to an unequal distribution of food, almost $11 \%$ of people face food insecurity (FAO, 2018). A reduction in food waste, while maintaining current production levels, might therefore increase food availability. Food insecurity does not only exist in developing countries; in the Netherlands, $14.1 \%$ of households were living on an income less than $120 \%$ of the social minimum in 2017 (CBS, 2018). Moreover, 80.000 people rely on Dutch food banks each year (Voedselbank-Nederland, 2018).

Food consumed in Europe accounts for $20-30 \%$ of the greenhouse gas emissions for all products consumed in this region (Scholz et al., 2015). As one third of all food produced is wasted, the environmental impact of food waste is substantial. By wasting edible food, the energy and resources spent in the production and distribution of these foods are wasted as well, as are their related environmental impacts (Gustavsson et al., 2011; Thyberg \& Tonjes, 2016). A study by Scherhaufer et al. (2018) shows the environmental impact of the European food waste constitutes $15.7 \%$ of the total global warming potential (GWP) generated by the food system in the EU. Meat and dairy products contribute much more than fruit and vegetables to the total GWP of the food system; therefore, wasting these products results in a higher environmental burden.

\subsection{Food waste by retailers}

Although the share of food waste at the retailer stage comprises just $5 \%$ of the total, there are several reasons why it is important to address retailers' food waste. At supermarkets, 
the products of many different food supply chains are brought together and distributed to many different consumers. Due to this interaction between the upstage actors of the supply chain (e.g. the food producers) and the consumer, the retailer influences a large part of the food supply chain, providing them with a great potential to reduce food waste (Parfitt et al., 2010; Gruber et al., 2016). Moreover, the retailer is the last stage of the food supply chain for which logistic interventions can be taken to reduce the food waste. In several European countries, legislation has already been introduced to reduce the amount of food waste at the retailer. In both France and Italy, the redistribution of food wasted by retailers is enforced by law (Cicatiello et al., 2017).

Many different products are wasted at retailers; with bakery, vegetables and fruit products representing the most commonly discarded items. The least food waste occurs for products with very long shelf lives, such as canned or frozen foods (Stenmarck et al., 2011). Not every retailer produces similar amounts of waste; in the few available studies considering retailer size, small stores were found to have a higher relative waste level than larger supermarkets (Gustavsson \& Stage, 2011; Beretta et al., 2013). Moreover, higher waste levels were identified for organic products than for conventional products (Eriksson et al., 2014). The low turnover of these products gives a relatively high uncertainty over the demand, which increases the risk to retailers of having enough, but not too much, of each product in stock.

Several studies show the imbalance between waste quantities and the economic and environmental impact of wasted food. In a study on the recovery of waste generated in an Italian supermarket, large quantities of bakery products were recovered, while the quantity of recovered meat was much lower. In contrast, the environmental impact of these meat products is much higher than those of the bakery products, despite the smaller quantities involved (Cicatiello et al., 2016). In another study on food waste at an Italian hypermarket, $34 \%$ of the food waste consisted of fruit and vegetables, which only accounted for $20 \%$ of the economic value of the wasted food (Cicatiello et al., 2017). A study by Scholz et al. (2015) found that 3.5\% of the food waste mass was composed of meat products, but it accounted for $29 \%$ of the carbon footprint of the waste of this retailer. The need to reduce food waste is therefore greater for certain products from both an environmental and economical perspective.

There are many causes of food waste at the retailer, but three main sources can be identified (Teller et al., 2018):

1. Consumer in-store behaviour and consumer demand

2. Store management, i.e., replenishment and assortment decisions

3. Product shelf life 
Consumers can be very selective about which products they buy at the store. They tend not to purchase products with an odd shape or visible damages (Cicatiello et al., 2016). Furthermore, consumers prefer fresh products to older ones; thus, they tend to leave products close to their expiration date on the shelf and purchase ones with a longer remaining shelf life (Tsiros \& Heilman, 2005; Teller et al., 2018). Moreover, consumer demand at the store is unpredictable, as it is influenced by many other factors, such as the weather, store promotions or store layout (van Donselaar et al., 2006).

In the case of highly perishable products, the fluctuation in consumer demand increases the complexity of replenishment. To cope with varying consumer demand, retailers tend to overstock to ensure a high service level for the consumer; however, this increases the risk of food waste (Cicatiello et al., 2016, 2017). A Scandinavian study suggested the need for improved replenishment at the retailer to reduce food waste levels at supermarkets (Stenmarck et al., 2011).

The third main cause for food waste is the shelf life of the product, which is also linked to the first two. Both consumer behaviour and store management have less of an impact on food waste for products with a longer shelf life.

\subsection{Possible interventions to reduce food waste at the retailer}

According to Papargyropoulou et al. (2014), prevention is preferable to other options for the reduction of food waste, such as re-use or the conversion to animal feed (see Figure 1.2). If the prevention of food waste is not possible, food waste can be recycled, composted or used to generate energy. The least preferable option is to dispose of food waste in landfill.

Despite the importance of reducing food waste by retailers, the literature dealing with retail operations in terms of food waste is limited. Although many suggestions for reducing food waste have been proposed, the focus of most studies is on the prevention of out-ofstock situations, shelf availability and profit levels; the costs of overstocking and waste are only included to a limited extent (Teller et al., 2018).

The number of publications considering inventory management for (highly) perishable products has risen over the years. One of the first reviews on this topic was written by Nahmias (1982), which was followed by many others such as those by Goyal \& Giri (2001), Bakker et al. (2012) and Janssen et al. (2016). Although more studies were analysed in these more recent reviews than in the past, the number of studies considering multi-item inventory management is still limited, despite these settings being the most closely related 


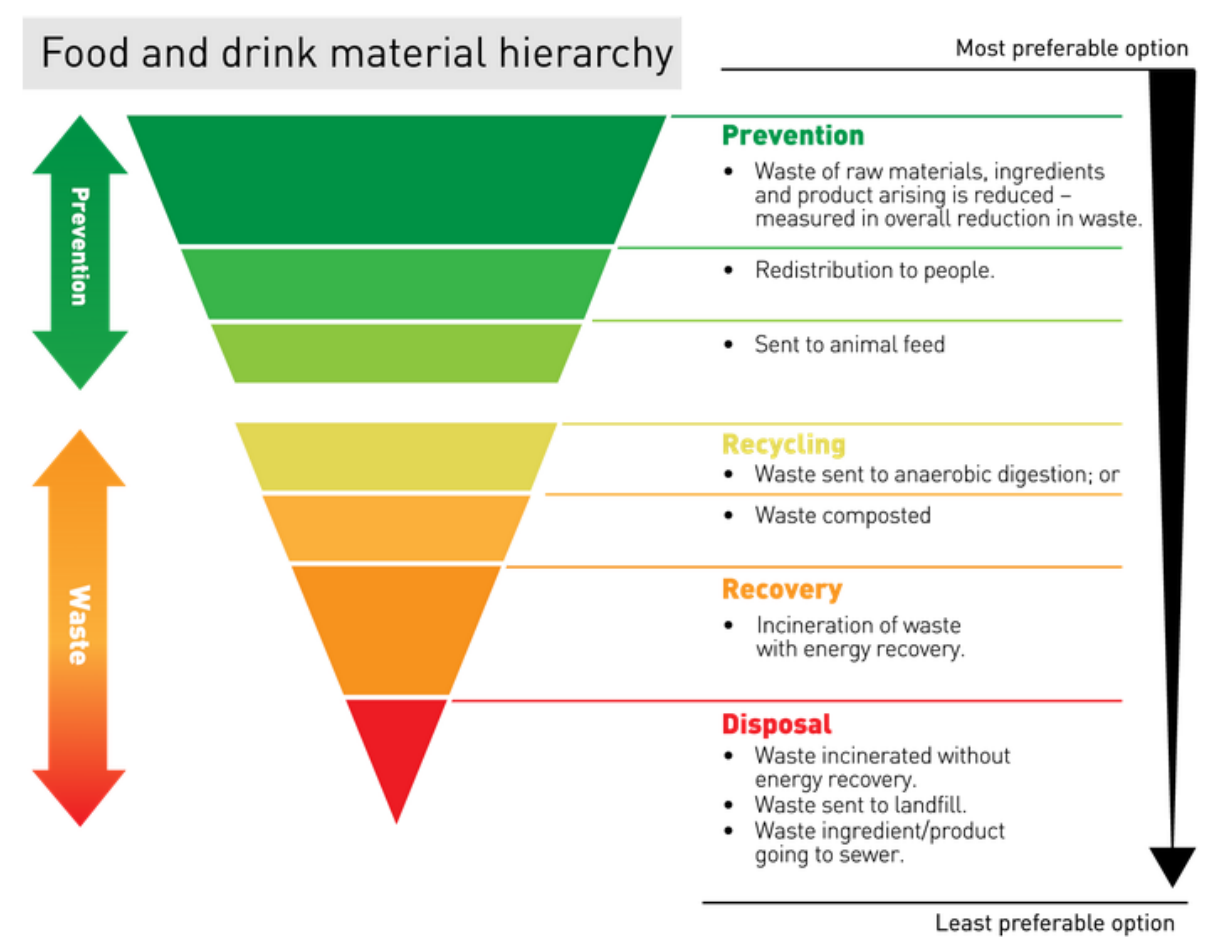

Figure 1.2: Food waste hierarchy (WRAP, 2019)

to reality (Bakker et al., 2012; Janssen et al., 2016). Studying realistic retailer settings is important to further explore food waste reduction, prevention or re-distribution.

Interventions to reduce food waste at the retailer level can be classified into three groups: marketing, logistical and technical interventions (Tromp et al., 2016). Marketing related interventions focus on increasing the turnover of products by increasing or influencing consumer demand. Studies show that prices and promotions (Stenmarck et al., 2011) or the assortment of products available influence consumer demand (Kok, 2003; Hübner et al., 2016). Logistical interventions relate to the reduction of time products spend in the supply chain before they are purchased by the consumer. Possible interventions can be made on lead time reductions (Tromp et al., 2016) or by applying stock-age based ordering (Haijema, 2014). Technical solutions to reduce food waste focus on the reduction of the quality decay of products. This can be obtained by improvements made to product packaging, or by optimising food handling or storage conditions in the supply chain (Buzby et al., 2011). Moreover, the accurate tracking of storage conditions and temperature along the supply chain of a product can facilitate the reduction of food waste (Ketzenberg et al., 2015).

Besides the studies on retailer inventory management or possible inventions for to reducing food waste, several studies have empirically considered the generation of food waste at the retailer, analysing both the discarded food products and the redistribution of the surplus 
food (Lebersorger \& Schneider, 2014; Cicatiello et al., 2016). Although a considerable amount of food waste can be recovered and re-used by other parties, it is still a challenge to use these leftovers efficiently to fight poverty and reduce food waste (Alexander \& Smaje, 2008; Cicatiello et al., 2016). There are some studies available considering the redistribution of food waste to food banks; however, these studies are focussed on vehicle routing problems, rather than the effective use of the food (e.g. Ghoniem et al., 2012; Solak et al., 2014; Brock \& Davis, 2015).

Although some research has been performed on food waste at the retailer level, studies typically related to retail operations, often focussed on profit and product availability, and did not consider the interventions for reducing food waste suggested by other, more empirical, studies. Besides waste reduction, studies related to the re-use of retailer food waste are also limited.

\subsection{Research outline and aims}

There is an environmental, social and economic need to reduce the amount of food waste by retailers. Current practices in the retail sector should be improved to reduce the environmental impact of the food system and to improve the availability of food for those who face food insecurity. This should all be done while considering the economic status of the retailer. Furthermore, section 1.3 highlights the lack of scientific research related to both the reduction of food waste at the retailer, and the use of food donations. To address these issues, the aim of this thesis is defined as follows:

Overall research aim: To analyse the impact of several interventions for reducing food waste at the retailer level from an Operations Research perspective.

As explained in Section 1.2, there are three main causes of food waste by retailers. Possible interventions to reduce food waste or re-use food leftovers at the retailer level are described in section 1.3. Figure 1.3 shows the conceptual framework used in this thesis, highlighting the three main causes of food waste; the consumer, the store management and the shelf life of the products. All these causes influence the amount of food waste at the retailer. In this thesis, interventions targeting the reduction or re-use of food waste by impacting the three main causes are analysed for their impact to reducing retailer food waste. As there is an imbalance between the amount of food waste $(\mathrm{kg})$ and the economic (or environmental) value of the wasted products, this thesis also considers both food waste quantity at the retailer as well as the economic value. The interventions not only affect food waste at the retailer level, but they also have an impact on the profit and service levels provided 
by these companies, which are both important performance measures for the retailer; therefore, these indicators are also included in this thesis.

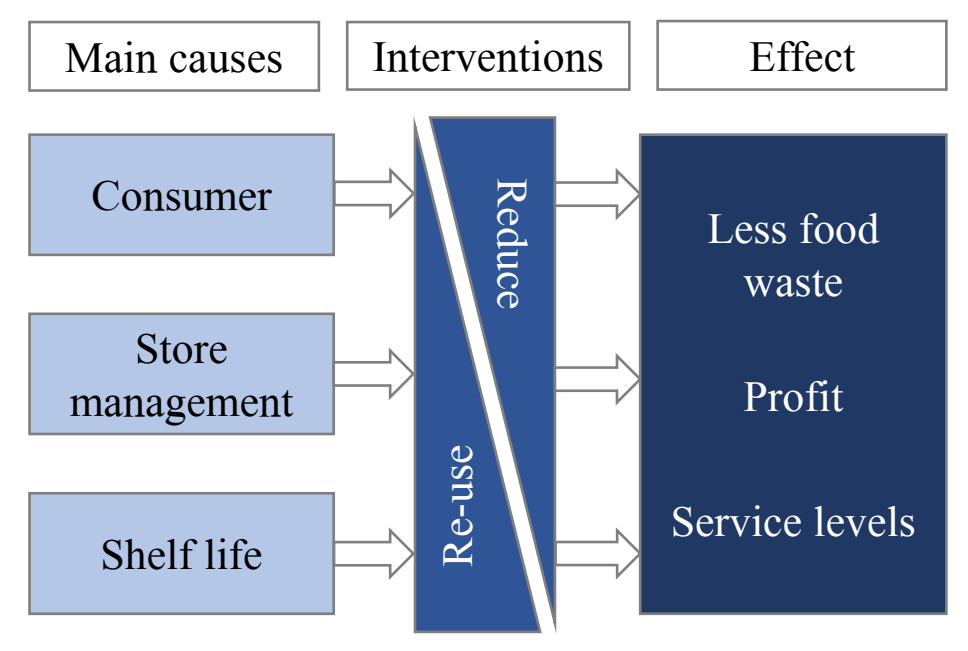

Figure 1.3: Research framework of this thesis

In Chapter 2, both discounting and dynamic shelf life are studied as intervention for reducing food waste at the retailer. Discounting almost expired products influences consumer behaviour, triggering the purchase of older, discounted products instead of the fresher alternatives. A dynamic shelf life is a shelf life based on the actual quality of the product rather than a fixed date that is equal for all products. Moreover, the replenishment of the products is optimised to further reduce food waste and improve profits for the retailer.

Product substitution is studied as an intervention in Chapter 3. It is known that consumers often buy a substitute product if their preferred item is out of stock. By including the substitution behaviour of consumers, the replenishment decision for a two-product case is optimised. This optimisation focusses both on increasing profits and reducing food waste, taking service level into account.

Retailers sell usually many more than two products. Therefore, in Chapter 4 the problem is extended to a multi-product case with multiple substitution attempts considering both the replenishment and the assortment decisions are considered. The focus of this chapter is to provide a solution framework able to deal with the complexity of combined inventory decisions for multiple products. Due to the complexity arising with this extension to multiple products, a single period model is used.

Although food waste can be reduced at the retailer, it is impossible to reduce this amount to zero. It is therefore important to consider ways to re-use food leftovers. As mentioned before, a limited number of studies have addressed this topic; therefore, in Chapter 5 , a study of the use of donated surplus food by a charity organisation is presented. Al- 
though this concept is studied from the perspective of a soup kitchen, the store management at the retailer and the product shelf life has a great effect on the possibility to use food leftovers. Moreover, in this chapter, contracts between the retailer and soup kitchen regarding the amount of donated food are included.

The thesis finishes with Chapter 6, in which the results of individual interventions are discussed. Furthermore, the link between the interventions and the potential for their combination are discussed. The discussion in the last chapter focusses on the implications of the studied interventions in a broader supply chain perspective, and on the methodological contributions. The thesis ends with suggestions for further research. 



\section{Chapter 2}

\section{Discounting and dynamic shelf life to reduce fresh food waste at retailers}

This chapter is published as:

Buisman, M. E., Haijema, R., \& Bloemhof-Ruwaard, J. M., (2019). Discounting and dynamic shelf life to reduce fresh food waste at retailers. International Journal of Production Economics, 209. doi: 10.1016/j.ijpe.2017.07.016 


\section{Abstract}

Approximately 89 million of tonnes of food is wasted every year in the EU along the whole food supply chain. The reasons for food waste by retailers include inappropriate quality control, overstocking and inaccurate forecasting. This study shows that food wasted by retailers can be reduced by discounting old products or by applying a dynamically adjustable expiration date (in other words dynamic shelf life (DSL)). We developed a simulation based optimization model to optimize the replenishment and discounting policy of a retailer who sells meat products. DSL outperforms a fixed shelf life (FSL) in terms of profit, waste, shortages and food safety. Furthermore, replenishment quantities can be higher. The benefits of DSL are greater when demand is low or when the shelf life of products is short. Discounting is a successful strategy to reduce food waste for both FSL and DSL. DSL without discounting is more effective than FSL with discounting. Combining DSL and discounting, allows for a further reduction of food waste. 


\section{Discounting and Dynamic Shelf Life}

\subsection{Introduction}

Food waste is a major problem for society. Approximately 89 million of tonnes is wasted in the EU every year (Monier et al., 2010). The most common causes of perishable food waste at a retailer are overstocking, consumer behaviour, inappropriate quality control and product handling (Wang \& Li, 2012; Whitehead et al., 2011). Therefore, an inventory management strategy and more focus on consumer behaviour are needed at the retailer. Products close to the use-by date are perceived as products with lower quality by consumers and are therefore less favourable to purchase (Tsiros \& Heilman, 2005). Discounting is a well-known technique to convince consumers to buy less favourable products and to reduce food waste.

Another way to reduce food waste is to better predict product quality and according adjust the shelf life (or use-by date) dynamically. Products with a maximal shelf life of less than 2 weeks are considered to be perishable products. For most of these products (e.g. meat, fish, dairy) it is obligatory to determine a use-by date and print it on the product packaging. The time between production and the use-by date is called shelf life. For highly perishable products, the shelf life is determined by producers and is often set rather conservatively to ensure food safety (Soethoudt et al., 2012). Conservative shelf life setting can cause unnecessary waste at retailers and increases when consumers are selective about the use-by dates or if demand varies a lot. It is expected that a DSL can reduce the amount of unnecessary waste. DSL is defined as a shelf life that can be adjusted to the actual quality of the product, either by adjusting the date or by indicating the quality of a product with a different technique, such as Time-Temperature Indicators (TTI). The latter has already proven to be beneficial in stochastic environments (Herbon et al., 2012). An extra advantage of a DSL is that the products that are sold are safer. The conventional approach of setting a FSL allows products to spoil before they reach the use-by date. This research evaluates the benefits of DSL for fresh meat products because fresh meat is highly perishable. Meat products are spoiled when bacterial counts are too high, and therefore food safety can be at stake when products pass their use-by date (Bruckner, 2010). To reduce food waste and ensure safe products we will study the effect of DSL and discounting on profit, waste, shortage and the replenishment quantity for a perishable product at a retailer. The effect of discounting on a retailer's performance is well studied, (e.g. see Elmaghraby \& Keskinocak, 2003; Lin et al., 2016; Transchel \& Minner, 2009; Zhao \& Zheng, 2000). However, some researchers make assumptions which do not hold for supermarkets (see Bakker et al., 2012; Chung \& Lin, 2001). On the other hand, the effect of DSL on a retailer's performance is hardly studied, nor is the combined effect of DSL and discounting. Both have an effect on the retailer performances however it is not yet known which of the two options is the most effective or how effective the 
combination of the two is. In addition, we will study the effect of discounting and DSL on the optimal replenishment quantities. Existing studies on DSL do not study the effect on the replenishment strategy. The effect of discounting on the replenishment strategy is only studied in the context of FSL (Farughi et al., 2014; Lin et al., 2016; Liu et al., 2008; Qin et al., 2014). It is unknown how the replenishment strategy of a retailer will be affected when DSL or the combination of DSL and discounting is applied. In this study we will fill these research gaps by evaluating the effect of discounting and DSL on the replenishment of a retailer and on its performance in terms of waste, profit, shortages and product quality. Discounting and DSL will be studied separately as well as combined. To study the effect of discounting and DSL on the replenishment, discount levels and replenishment quantities are optimized integrally.

In section 2.2 relevant literature on discounting and DSL is discussed. Section 2.3 presents the models used in this research. In section 2.4 we numerically investigate the effectiveness of DSL, discounting and their combination for a variety of experiments. Section 2.5 closes the chapter with conclusions and discussions.

\section{$2.2 \quad$ Literature}

To position this chapter, we discuss the literature related to discounting and DSL. We limited ourselves to articles published since 2008, in order to present an overview of recent developments. In Table 2.1 the most relevant articles are listed, which are obtained using search keywords: perishables AND [dynamic pricing OR discount OR dynamic shelf life]. Articles are assessed on several criteria; first if they include dynamic pricing or discounting and if prices are based on quality. Then how shelf life is set, fixed or dynamic and if demand is modelled deterministic or stochastic. When optimization is included, the focus of optimization is given. The last columns in the table explain which part of the supply chain (SC) is taken into account and if simulation is used. In 2.2.1 research on dynamic pricing is described in more detail and in 2.2.2 the literature about DSL. The other columns of the table are incorporated in those paragraphs. As indicated in the last row of Table 2.1, this chapter differentiates itself from most existing literature by including a DSL instead of a FSL. The few articles found that do include DSL, do not include the optimization of discount levels and replenishment quantities as we have.

\subsubsection{Discounting and dynamic pricing}

Discounting or determining an optimal price is a topic well studied in literature. Several good reviews are available such as Elmaghraby \& Keskinocak (2003) and Bakker et al. 
Table 2.1: Literature Review

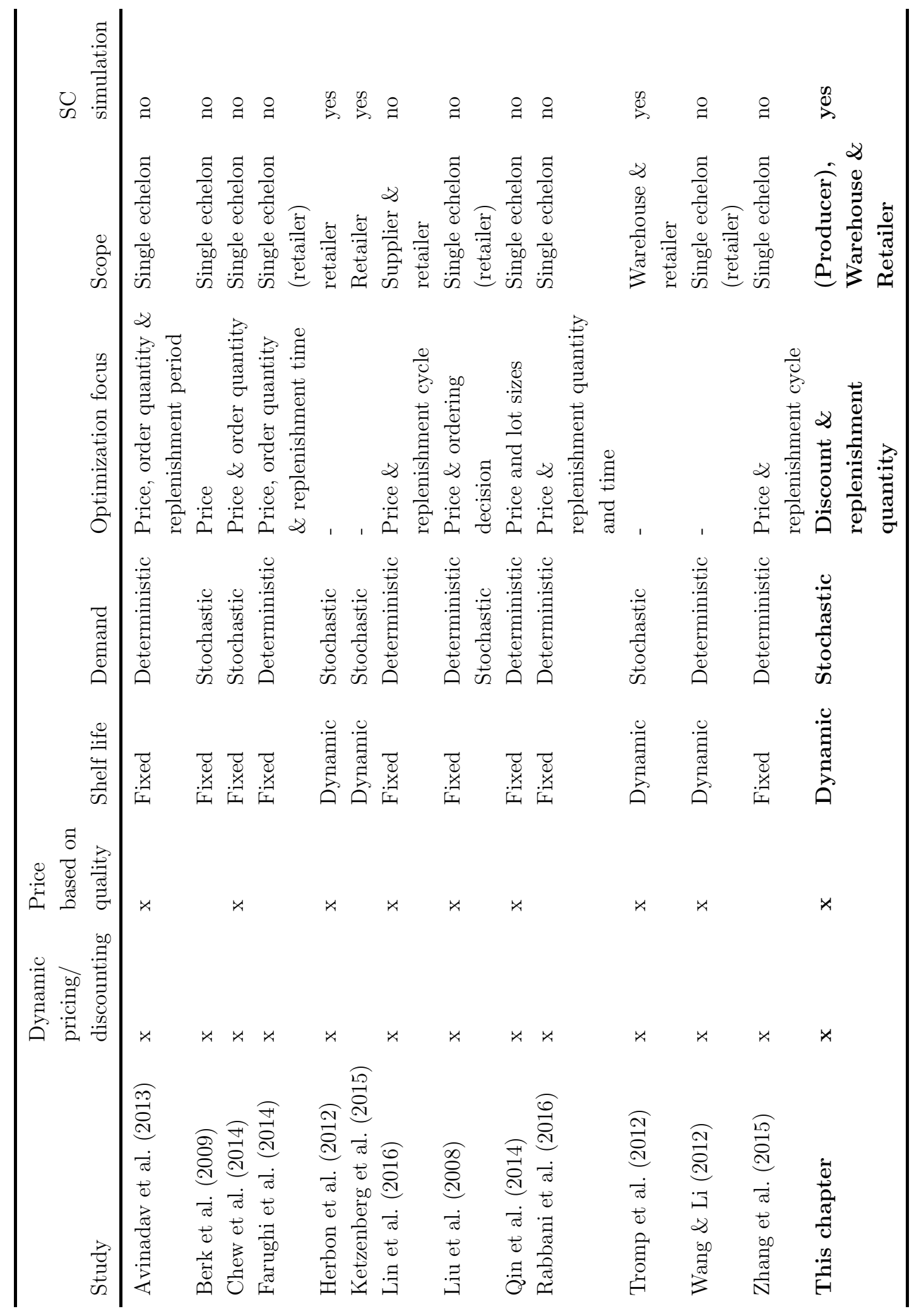


(2012). As Table 2.1 indicates, most of the reviewed articles include dynamic pricing or discounting. Profit is maximized by determining the optimal price and/or optimal replenishment (policy) (Farughi et al., 2014; Rabbani et al., 2016; Zhang et al., 2015). Price determination by product quality is done by Avinadav et al. (2013),(Chew et al., 2014), Qin et al. (2014) and Lin et al. (2016). Most researchers that focus on discounting/dynamic pricing developed an optimization model to evaluate a single, deteriorating product with a price dependent and deterministic demand. (Berk et al., 2009) did not include replenishment policies in their research but investigated the effect of costs that come with adapting the price. Next to that, they are one of the few who incorporated stochastic demand. In order to solve the optimization problem they developed a heuristic. Liu et al. (2008) developed an optimization model to determine optimal price and ordering decision. They first developed the model for deterministic demand and later extend it to stochastic demand. Demand is price and quality dependent, and they apply an RFID tag to indicate food quality. Chew et al. (2014) also used stochastic demand. They evaluated a product with a multi-period life- time and allowed substitution between products of different age categories. For a life-time of 2 periods they show that an optimal price can be obtained analytically. For life-times higher than 2 periods a heuristic is developed to find the optimal solution. The results show that profit increases when price and order quantity for both products are determined together. Avinadav et al. (2013) developed an optimization model where demand is not only price dependent but also dependent on remaining shelf life. Although the scope of each study is slightly different, the conclusions are closely related. The conclusions of the studies generally show that the costs of price changes, speed of deterioration and consumer behaviour influences the optimal price (policy) (Berk et al., 2009; Lin et al., 2016; Qin et al., 2014).

\subsubsection{Dynamic shelf life}

Only a few researchers implement a DSL, shelf life based on the quality status of the product. Tromp et al. (2012) and Wang \& Li (2012) implemented DSL in combination with discounting. Both use a simulation model to determine the effect of a DSL compared to a FSL. Tromp et al. (2012) models a pork supply chain and incorporates food safety by modelling food quality with a microbiological growth model. In their research they include a stochastic consumer demand divided in FIFO and LIFO demand. Without discounting, this ratio is fixed, but when a discount is applied, it is assumed that more consumers will buy FIFO. They show that a DSL is a promising concept compared to a FSL when evaluating opportunity losses that occur due to stock-outs and waste. Wang \& Li (2012) developed a similar model, but modelled food quality more generally and therefore did not include food safety. Furthermore, they work with deterministic settings. They show that setting prices according to a dynamically identified food quality can improve the 


\section{Discounting and Dynamic Shelf Life}

retailers' benefits and reduces waste at retailers. Herbon et al. (2012) evaluate the effect of using a TTI on retailers' profit for a luxurious fish product. They developed a nonlinear stochastic model, which they solve by simulation. Demand is price dependent and stochastic. They evaluated four levels of discount (from 0 till 20\%) and two types of TTI (simple and cheap or sophisticated and more expensive). They conclude that applying a simple TTI increases profit. A sophisticated TTI can decrease profit because the cost reduction is less than the price of the tag. Furthermore, they found that applying discounts is beneficial. Ketzenberg et al. (2015) focus on the value of using a TTI by formulating a Markov Decision Process, solved with a heuristic. They measure the value of information (VOI) of the TTI as the reduction in average costs when information is available. A simulation study is performed to evaluate the VOI. The results give a high VOI for the majority of the experiments. This implies a large uncertainty present in the model that will affect the retailers' performance.

\subsubsection{Research gap}

The literature review shows that for dynamic pricing the effect on replenishment is well described, although, not always under assumptions that hold for a retailer. Most of the studies deal with deterministic demand, which is preferable from a mathematical perspective. Deterministic demand might be applicable for situations where demand is high and variability low however when dealing with real life situations at a grocery store, stochastic demand is more realistic. The effect of a DSL on the replenishment (quantity) has not yet been incorporated as far as we know. Neither is the effect of a demand shift (from LEFO to FEFO) studied when applying a discount. Almost all the research mentioned above assumes an increase in demand when price decreases. There does not seem to be a chapter that compares discounting and DSL, and their impact on food waste, shortages, profit and replenishment levels.

\subsection{Method}

In order to analyse the effect of DSL and discounting on profit, waste, shortages and product quality, simulation-based optimization is applied. This allows the SC to be modelled at the right level of detail for monitoring quality decay at both the retailer and the distribution centre (DC). Factors, such as uncertain demand, temperature fluctuations and order lead time can be included as well. 


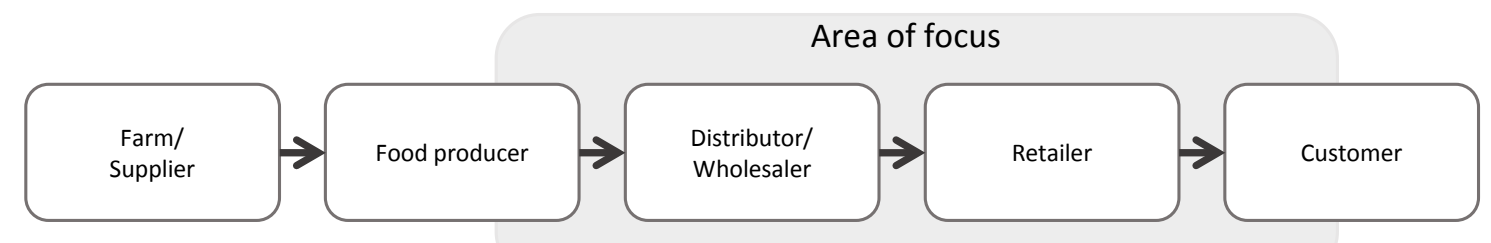

Figure 2.1: Research scope of meat supply chain

\subsubsection{Simulation model}

The core of the simulation model is an inventory model, describing part of a meat supply chain (Figure 2.1). The main focus of the model is a retailer which is supplied by a DC. The DC is supplied by a production company. The DC serves multiple retailers at the same time but the evaluation is focussed on one retailer. The consumer is within the scope of this research, but only at the moment of purchase. What happens after purchasing the product is out of the research scope. Transport by truck will take place between the processing company and the DC and between the DC and the retailer. Next to the inventory model, a microbiological growth model is included to track the quality of the products. The modelling of the product is done in batches based on their remaining shelf life at the DC and retailer however, consumers purchase a single product out of those batches.

\section{In- and outputs}

The simulation model evaluates and compares different scenarios in terms of average profit per week, waste, shortages and microbiological counts of sold products. Waste is chosen because it is the main focus of this research. In this section it is explained how waste occurs at the retailer for scenarios with fixed and DSL. Moreover, profit calculations and shortages are counted as the percentage of demand that cannot be fulfilled are given. Finally, the microbiological count is explained. Profit margin, selling price and consumer demand are used as inputs, to calculate waste and profit. We need the initial contamination of the product after packaging and the temperature in the supply chain, to obtain the average microbiological count of sold products.

\section{Microbiological model}

A microbiological growth model is used to determine the quality of the products. For fresh meat products shelf life is mainly determined by bacterial growth. The (modified) Gom- 


\section{Discounting and Dynamic Shelf Life}

pertz curve is one of the most used model in modelling microbiological growth (Bruckner, 2010; Tromp et al., 2012). In this research an adapted version of the Gompertz curve is used, based on the research of Tromp et al. (2012). This adapted Gompertz curve can deal with temperature changes more easily than the original Gompertz curve. $N_{\tau}$ is the microbiological count of a product that has been produced $\tau$ days ago and kept since then at temperature $\mathrm{T}\left(\right.$ in $\left.{ }^{\circ} \mathrm{C}\right)$.

$$
N_{\tau}=A+C \cdot e^{-e^{-B_{T} \cdot\left(\tau-M_{T}\right)}}\left[\log _{10} \mathrm{cfu} / \mathrm{g}\right]
$$

Where A, B, C and M product specific parameters. Parameters B and M are temperature dependent according to:

$$
\begin{gathered}
B_{T}=\alpha_{B} e^{\beta_{B} \cdot T} \\
M_{T}=\alpha_{M} e^{\beta_{M} \cdot T}
\end{gathered}
$$

The Gompertz model is a continuous time growth model, i.e. $N_{\tau}$ is a continuous function of $\tau$. However, in the simulation model the microbiological count of the products are updated at discrete points in time, e.g. at the start and end of a process like transshipment. During time intervals in between time points the temperature is assumed to be constant. Temperature changes are modelled at these time points. At the end of the day, microbiological growth for every batch in stock is calculated as follows.

At the end of the day $t$ product batch $r$ was exposed to a constant temperature $\mathrm{T}$ during $\tau_{\text {step }}$ units of time. At its last update, $\tau_{\text {step }}$, time units ago, the cell count was $N_{t r}$. That value corresponds to the point $\tau=\phi$ at the Gompertz curve. The value of $\phi$ follows from the inverse of equation (2.1).

$$
\tau=\phi=\frac{\log \left(-\log \left(\frac{N_{t r}-A}{C}\right)\right)}{-B_{T}}+M_{T}
$$

At the start of the next day, $t+1$, batch $r$ will be labelled batch $r-1$; the bacterial count on a product is:

$$
N_{t+1, r-1}=A+C \cdot e^{-e^{-B_{T}\left(\phi+\tau_{s t e p}-M_{T}\right)}}\left[\log _{10} \mathrm{cfu} / \mathrm{g}\right]
$$

Besides an update on microbiological count every day, updates also take place when products are transshipped. 


\section{Producer and $D C$}

The simulation model starts at Monday $(t=0)$, at the moment a producer packages the product (Figure 2.1). We assume that the DC orders three times a week, on Monday, Wednesday and Friday at the end of the day. The producer delivers at the DC within $12 \mathrm{~h}$, before the retail outlets open. Effective lead time is therefore zero and incoming products at the DC will have a shelf life of $m-1$ days. Delivery of the producer to the DC is described with an order-up-to level:

$$
Q_{t}^{D C}= \begin{cases}S^{D C}-\sum_{r=1}^{m-1} I_{t r}^{D C} & \text { if } \bmod (\mathrm{t}, 7)=\{0,2,4\} \\ 0 & \text { else }\end{cases}
$$

Where $Q_{t}^{D C}$ is the delivery quantity and $I_{t r}^{D C}$ the number of products with remaining shelf life $r$ still in stock at the DC upon ordering at the end of day $t$. The DC serves $e$ retailers, each with a Poisson distributed daily demand with mean $\mu$ products. The DC places an order every $R=2$ working days. The demand at the DC over the next $R$ working days has a mean demand $e \mu R$ and a standard deviation of $\sqrt{e \mu R}$. The order-up-to level of the DC, $S^{D C}$, is set by:

$$
S^{D C}=e \mu R+z^{D C} \cdot \sqrt{e \mu R}
$$

Where $z^{D C}$ is a safety factor, which will be large enough such that enough products are available at the DC. The order policy for the focal retailer is explained later on. The DC sells products to retailers with a FEFO policy and when products are delivered of different age categories, they are equally distributed among the retailers. For example, if the DC meets $60 \%$ of the total demand by products from the 'oldest' batch and $40 \%$ from the 'next-to-oldest' batch, then $60 \%$ of the focal retailer's demand is met by products from the oldest batch and $40 \%$ by products of the next-to-oldest batch. Similarly, shortages are equally spread over the retailers by the ratio of their demands.

Inventory of the DC is updated at the end of each period.

$$
I_{t+1, r-1}^{D C}= \begin{cases}I_{t r}^{D C}-P S_{t r}^{D C}+Q_{t}^{D C} \delta(r=m-1)-W_{t r}^{D C} \delta(r=1) & \text { for FSL } \\ I_{t r}^{D C}-P S_{t r}^{D C}+Q_{t}^{D C} \delta(r=m-1)-W_{t r}^{D C} \delta\left(N_{t r} \geq \eta_{\text {waste }}\right) & \text { for DSL }\end{cases}
$$

Where, $P S_{t r}^{D C}$ are the products sold by the DC at period t with remaining shelf life $r, \delta$ is a Kronecker delta where $\delta(x)=1$ if $x$ is true, and 0 otherwise and $W_{t r}^{D C}$ the products wasted in period $\mathrm{t}$ with a remaining shelf life $\mathrm{r}$ calculated in a similar way as for the retailer, explained before. 


\section{Discounting and Dynamic Shelf Life}

\section{Ordering policy focal retailer}

The retailer is open 6 days a week from Monday to Saturday. The number of days passed since the start of the simulation is indicated by index $t$. The related weekday is indexed $d=\bmod (t, 7) \in\{0=$ Monday $, 1,2, \ldots, 6=$ Sunday $\}$. At the beginning of day $t$ the retailer places an order $\left(Q_{t}^{\text {Ret }}\right)$ at the DC excluding Sundays. Products are replenished with a weekday dependent order-up-to-level $S_{d}^{\text {Ret }}$. Products are ordered in multiples of a pack G. Furthermore the order size depends on the total number of products in stock $\sum_{r=1}^{m} I_{t r}^{R e t}$ at the moment of ordering (at the start of day $t$ ), and the estimated amount of products to be wasted at the end of a period $\left(E W_{t}\right)$. EW $W_{t}$ is the amount of products with a remaining shelf life of one day subtracted by the expected FEFO sales during that day, which is a fraction of the mean demand $\mu_{d}$ on a weekday $d$. To keep the rule simple to use, we do not subtract the part of demand of LEFO consumers that is met from that category. Thus the order quantity set by the retailer at day $t$ is:

$$
Q_{t}^{R e t}= \begin{cases}{\left[\frac{S_{d}^{R e t}-\sum_{r=1}^{m} I_{t r}^{R e t}+E W_{t}}{G}\right] \cdot G} & \text { if } \bmod (\mathrm{t}, 7) \in\{0, . ., 6\} \\ 0 & \text { otherwise }\end{cases}
$$

Where the squared brackets indicate $Q_{t}^{R e t}$ is rounded to the nearest multiple of pack size $G$

$$
E W_{t}= \begin{cases}\max \left[0, I_{t 1}^{R e t}-(1-a) \cdot \mu_{d}\right] & \text { if FSL } \\ \max \left[0, \sum_{r=1}^{m} I_{t r}^{R e t} \delta\left(N_{t r} \geq \eta_{\text {waste }}\right)-(1-a) \cdot \mu_{d}\right] & \text { if DSL }\end{cases}
$$

Consumer demand and withdrawal at retailer

Consumer demand $\left(D_{t}\right)$ is assumed to be stochastic, and Poisson distributed with weekday dependent mean demand $\mu_{d}$ and standard deviation $\sigma_{d}=\sqrt{\mu_{d}}$. The meal weekly demand at a retailer is $6 \mu$, and on average a fraction $f_{d}$ of the week demand occurs on weekday d. Thus, we have:

$$
\mu_{d}=f_{d} \cdot 6 \mu
$$

Total consumer demand is separated in consumers who buy FEFO and LEFO. It is assumed that LEFO consumers will buy products before FEFO consumers arrive, as they are pickier about product quality and therefore might put more effort into getting products earlier. Division is done as follows:

$$
\begin{gathered}
D L_{t}=D_{t} \cdot a \\
D F_{t}=D_{t}-D L_{t}
\end{gathered}
$$


With $D L_{t}$ is the LEFO demand and $D F_{t}$ is FEFO demand and a the fraction of total demand which is LEFO. When a discount is applied it is assumed that the ratio between LEFO and FEFO consumers shift more towards FEFO consumers. Based on the discount percentages $(x)$ a similar percentage of the LEFO consumers will pick the discounted product and therefore into FEFO consumers. The new LEFO demand $D L_{t}^{D i s c}$ is calculated by

$$
D L_{t}^{\text {Disc }}=D L_{t}-\min \left\{x \cdot D L_{t}, a \cdot I D i s c_{t r}^{\text {Ret }}\right\}
$$

Where, IDisc $c_{t r}^{\text {Ret }}=I_{t 1}^{\text {Ret }}$ the products with a discount at time $t$ when FSL applies and $I D i s c_{t r}^{\text {Ret }}=I_{t r}^{\text {Ret }} \delta\left(N_{t r} \geq \eta_{\text {discount }}\right)$ the products with a discount at time $t$ when DSL applies.

In the case of LEFO withdrawal, products picked by the consumer $\left(P L_{t r}^{R e t}\right)$ at period $t$ with remaining shelf life $r$, is the minimum of the products available of a batch $(t r)$ and the remaining demand which is unsatisfied from fresher batches. For $r=m, m-1, \ldots, 1$,

$$
P L_{t r}^{R e t}=\min \left\{I_{t r}^{R e t}, D L_{t}-\sum_{i=r+1}^{m} P L_{t i}^{R e t}\right\}
$$

In case of FEFO withdrawal products picked by a consumer at period $t\left(P F_{t r}^{\text {Ret }}\right)$ are the minimum of the remaining products in the batch and the remaining demand which is unsatisfied from older batches on the shelf, for $r=1, \ldots, m$,

$$
P F_{t r}^{R e t}=\min \left\{I_{t r}^{R e t}-P L_{t i}^{R e t}, D F_{t}-\sum_{i=1}^{r-1} P F_{t i}^{R e t}\right\}
$$

Wasting policy for FSL and DSL at retailer

At the end of the shelf life products are wasted. For a FSL this will happen when products have a remaining shelf life of one day left after closing the shop. The wasted products are:

$$
W_{t}^{\text {Ret }}=I_{t 1}^{\text {Ret }}-P L_{t 1}^{\text {Ret }}-P F_{t 1}^{\text {Ret }}
$$

With a DSL, the moment of wasting the product is determined by the amount of bacteria present on the product. When products have a higher bacterial count than $\eta_{\text {waste }}$ they will be wasted at the end of a day.

$$
W_{t}^{\text {Ret }}=\sum_{r=1}^{m}\left(I_{t r}^{\text {Ret }}-P L_{t r}^{\text {Ret }}-P F_{t r}^{\text {Ret }}\right) \cdot \delta\left(N_{t r} \geq \eta_{\text {discount }}\right)
$$




\section{Discounting and Dynamic Shelf Life}

In the final evaluation, waste is defined as the percentage of products bought by the retailer. At the end of the day, the inventory at the retailer is updated for the remaining shelf life and time period.

$$
I_{t+1, r-1}^{R e t}= \begin{cases}I_{t r}^{R e t}-P L_{t r}^{R e t}-P F_{t r}+Q S_{t r}^{R e t}-W_{t r}^{R e t} \delta(r=1) & \text { for FSL } \\ I_{t r}^{R e t}-P L_{t r}^{R e t}-P F_{t r}+Q S_{t r}^{R e t}-W_{t r}^{R e t} \delta\left(N_{t r} \geq \eta_{w a s t e}\right) & \text { for DSL }\end{cases}
$$

where: $Q S_{t r}^{R e t}$ are the incoming products at the retailer at time $t$ of age class $r$. Depending on the demand of all other retailers, and the inventory levels of the DC.

\section{Profit}

In this research profit is defined as revenues minus purchasing and holding costs. Fixed ordering costs are neglected as perishables at supermarkets are usually replenished daily or transport costs are shared over many products (Haijema \& Minner, 2016).

$$
\begin{gathered}
\text { Profit }_{t}=\sum_{r=1}^{m}\left(\left(P L_{t r}^{\text {Ret }}+P F_{t r}\right) \cdot p_{t r}-I_{t r}^{\text {Ret }} \cdot h-Q S_{t r}^{\text {Ret }} \cdot p \cdot(1-\pi)\right) \\
p_{t r}= \begin{cases}p(1-x) & \text { if } I D i s c_{t r}^{\text {Ret }}>0 \\
p & \text { if } I D i s c_{t r}^{\text {Ret }}=0\end{cases}
\end{gathered}
$$

Where, $p$ is the sales price at the retailer, $x$ the discount given, $\pi$ the profit margin at the retailer, and $h$ the holding costs per item, which is determined by $p, \pi$ and $\gamma$ (fraction) as follow:

$$
h=\gamma \cdot \frac{p}{1-\pi}
$$

\subsubsection{Optimization}

Optimization is carried out for two values, the safety factor $z$ and the discount level $x$. The optimization gives input values to the simulation model and can therefore be seen as a layer over the simulation model.

\section{Safety factor $(z)$}

The optimal $z^{*}$ value for the retailer is determined by maximizing profit. To determine the right safety factor a full enumerated search is done. Values tested for $z$ range from 0 to 3 with intervals of 0.1 . Optimization over $z$ is chosen as demand varies among days 
and therefore the order-up-to level will be different each day. The order-up-to level is calculated with the $z^{*}$ as follow:

$$
S_{d}^{\text {Ret }}= \begin{cases}\mu_{d}+\mu_{d+1}+z \sqrt{\sigma_{d}^{2}+\sigma_{d+1}^{2}} & \text { if } d \in\{0, . ., 4\} \\ \mu_{5}+\mu_{6}+\mu_{0}+z \sqrt{\sigma_{5}^{2}+\sigma_{6}^{2}+\sigma_{0}^{2}} & \text { if } d=5 \\ 0 & \text { otherwise }\end{cases}
$$

Note, in our case the retailer is not open on Sunday, hence $\mu_{6}=\sigma_{6}=0$.

Discount level (x)

Discounting will occur on the last day products can be sold. For FSL this will be at $r=1$, for DSL when microbiological count is $\geq \eta_{\text {discount }}$. When discount is applied, a range from $0 \%$ up to $100 \%$ is tested with intervals of $5 \%$. Then the optimal $x^{*}$ is determined for every discount level.

\subsection{Numerical results}

In this section, we investigate the effectiveness of DSL and discounting and evaluate performance at the retailer on profit, waste and shortage levels and microbiological count. Section 2.4.1 describes the design of experiments and the data used. From section 2.4.2 onwards results are listed and discussed.

\subsubsection{DoE and data}

Table 2.2 gives the parameters settings for 32 experiments. Scenarios differ according to profit margins, mean demand, shelf life (different product) and whether discounting applies or not. For all experiments the safety factor $\mathrm{z}$ is optimized. In experiment 19 to 32 , the impact of optimal discounting is investigated, as well as the moment of discounting and the effect of the LEFO-FEFO ratio. The scenarios and results are discussed in detail in section 2.4.3, 2.4.4 and 2.4.5.

\section{Shelf life setting}

Shelf life setting for FSL is based on the predicted growth of bacteria during the life span of a product. As temperature is the main influencer, producers have to estimate the SC temperature to set a shelf life. Producers who define the use-by date of products want 
Table 2.2: Design of experiments: (a) Scenario 1-5 (b) Scenario 6 and 7

\begin{tabular}{|c|c|c|c|c|c|}
\hline Scenario & Experiment & $\begin{array}{l}\text { Shelf life }(m) \\
\text { (Days) }\end{array}$ & $\begin{array}{l}\text { Profit } \\
\text { margin }(\pi)\end{array}$ & $\begin{array}{l}\text { Weekly } \\
\text { demand }(6 \mu)\end{array}$ & $\begin{array}{l}\text { Order DC } \\
\text { per week }\end{array}$ \\
\hline \multirow[t]{2}{*}{ 1. Basic } & 1 & Fixed (8) & $33 \%$ & 30 & 3 \\
\hline & 2 & Dynamic & $33 \%$ & 30 & 3 \\
\hline \multirow[t]{4}{*}{ 2. Profit margin } & 3 & Fixed (8) & $20 \%$ & 30 & 3 \\
\hline & 4 & Dynamic & $20 \%$ & 30 & 3 \\
\hline & 5 & Fixed (8) & $50 \%$ & 30 & 3 \\
\hline & 6 & Dynamic & $50 \%$ & 30 & 3 \\
\hline \multirow[t]{4}{*}{ 3. Demand } & 7 & Fixed (8) & $33 \%$ & 18 & 3 \\
\hline & 8 & Dynamic & $33 \%$ & 18 & 3 \\
\hline & 9 & Fixed (8) & $33 \%$ & 48 & 3 \\
\hline & 10 & Dynamic & $33 \%$ & 48 & 3 \\
\hline \multirow[t]{4}{*}{ 4. Different product } & 11 & Fixed (5) & $33 \%$ & 30 & 3 \\
\hline & 12 & Dynamic & $33 \%$ & 30 & 3 \\
\hline & 13 & Fixed (10) & $33 \%$ & 30 & 3 \\
\hline & 14 & Dynamic & $33 \%$ & 30 & 3 \\
\hline \multirow{4}{*}{$\begin{array}{l}\text { 5. Different order } \\
\text { moment DC }\end{array}$} & 15 & Fixed (8) & $33 \%$ & 30 & 2 \\
\hline & 16 & Dynamic & $33 \%$ & 30 & 2 \\
\hline & 17 & Fixed (8) & $33 \%$ & 30 & 6 \\
\hline & 18 & Dynamic & $33 \%$ & 30 & 6 \\
\hline Scenario & Experiment & $\begin{array}{l}\text { Shelf life }(m) \\
\text { (Days) }\end{array}$ & $\begin{array}{l}\text { LEFO } \\
\text { fractions }(a)\end{array}$ & \multicolumn{2}{|c|}{ Discount moment } \\
\hline \multirow{4}{*}{$\begin{array}{l}\text { 6. Moment of discount and } \\
\text { optimal discount }\end{array}$} & 19 & Fixed (8) & 0.40 & \multicolumn{2}{|l|}{$r=1$} \\
\hline & 20 & Dynamic & 0.40 & \multirow{2}{*}{\multicolumn{2}{|c|}{$\begin{array}{l}\eta_{\text {discount }}=4.7 \log \mathrm{cfu} / \mathrm{g} \\
r=2\end{array}$}} \\
\hline & 21 & Fixed (8) & 0.40 & & \\
\hline & 22 & Dynamic & 0.40 & \multicolumn{2}{|c|}{$\eta_{\text {discount }}=4.3 \log \mathrm{cfu} / \mathrm{g}$} \\
\hline \multirow{10}{*}{$\begin{array}{l}\text { 7. Consumer picking and } \\
\text { optimal discount }\end{array}$} & 23 & Fixed (8) & 1.00 & \multicolumn{2}{|c|}{$r=1$} \\
\hline & 24 & Fixed (8) & 0.75 & \multicolumn{2}{|l|}{$r=1$} \\
\hline & 25 & Fixed (8) & 0.50 & \multicolumn{2}{|l|}{$r=1$} \\
\hline & 26 & Fixed (8) & 0.25 & \multicolumn{2}{|l|}{$r=1$} \\
\hline & 27 & Fixed (8) & 0.00 & \multicolumn{2}{|c|}{$r=1$} \\
\hline & 28 & Dynamic & 1.00 & \multicolumn{2}{|c|}{$\eta_{\text {discount }}=4.7 \log \mathrm{cfu} / \mathrm{g}$} \\
\hline & 29 & Dynamic & 0.75 & \multicolumn{2}{|c|}{$\eta_{\text {discount }}=4.7 \log \mathrm{cfu} / \mathrm{g}$} \\
\hline & 30 & Dynamic & 0.50 & \multicolumn{2}{|c|}{$\eta_{\text {discount }}=4.7 \log \mathrm{cfu} / \mathrm{g}$} \\
\hline & 31 & Dynamic & 0.25 & \multicolumn{2}{|c|}{$\eta_{\text {discount }}=4.7 \log \mathrm{cfu} / \mathrm{g}$} \\
\hline & 32 & Dynamic & 0.00 & \multicolumn{2}{|c|}{$\eta_{\text {discount }}=4.7 \log \mathrm{cfu} / \mathrm{g}$} \\
\hline
\end{tabular}




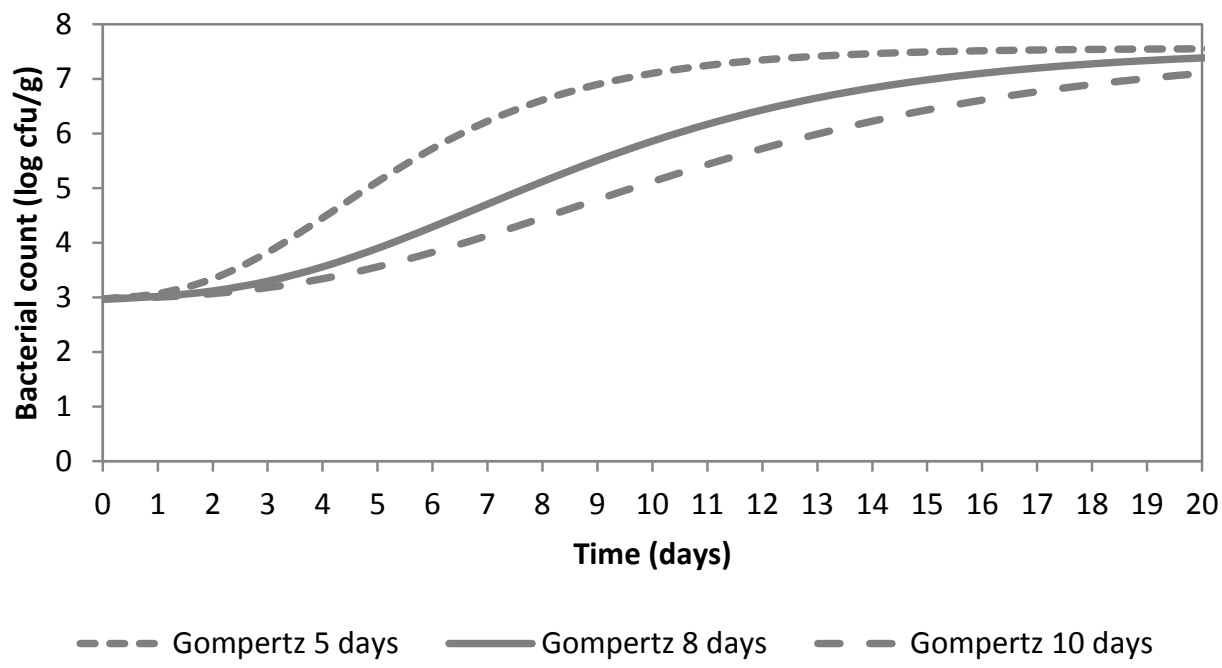

Figure 2.2: Example of increase in bacterial count according to modified Gompertz curve at $\mathrm{T}=5.5^{\circ} \mathrm{C}$

to ensure food safety and want to avoid selling products that are spoiled. Temperatures in the SC can vary considerably from the desired temperature, for instance while the products are unloaded from trucks. On average the $\mathrm{SC}$ temperature is around $4.5{ }^{\circ} \mathrm{C}$ (Tromp et al., 2012). To be safe and allow for some temperature variation we determine the use-by date for the meat product at a temperature of $5.5{ }^{\circ} \mathrm{C}$. Meat products are considered to spoil at a microbiological count of $\eta_{\text {unsafe }}=6 \log \mathrm{cfu} / \mathrm{g}$. In order to avoid selling products to consumers too close to that spoilage point, we use a limit of $\eta_{\text {waste }}=5.3$ $\log \mathrm{cfu} / \mathrm{g}$ at the retailer, in line with Tromp et al. (2012). At $5.5^{\circ} \mathrm{C} \eta_{\text {waste }}$ is reached after 8.45 days, therefore we use a shelf life of 8 days in the basic scenario for FSL. Figure 2.2 shows the development of bacterial count according to the modified Gompertz. The initial increase is low (lag phase), followed by exponentially growth and slows down when it reaches the upper limit. The tree curves relate to 3 different products (scenario 4) that have different growth times. In the plotted example, $\eta_{\text {waste }}$ is reached after a FSL of respectively 5,8 and 10 days at $5.5^{\circ} \mathrm{C}$ by multiplying $\tau$ in equation $(2.1)$ by a factor $(\zeta)$. $\zeta$ is calculated by dividing the new shelf life (in days) by the old shelf life.

For DSL we use the same limit for $\eta_{\text {waste }}$. For practical reasons we mimic the DSL in the simulation model by calculating with a maximum shelf life $(m)$ three times as high as used for FSL.

\section{Data}

In Table 2.3, the supply chain time and temperatures are given for the incorporated part of the SC, needed for the microbiological growth model. 


\section{Discounting and Dynamic Shelf Life}

Table 2.3: Time and temperature of supply chain based on Tromp et al. (2012)

\begin{tabular}{lll}
\hline Activity & Time (days) & Temperature distribution \\
\hline Transport from processing company to DC & 0.208 & Normal $\left(\mu=2^{\circ} C, \sigma=0.5^{\circ} \mathrm{C}\right)$ \\
Unloading at DC & 0.031 & Normal $\left(\mu=2^{\circ} C, \sigma=0.5^{\circ} \mathrm{C}\right)$ \\
At DC & $\begin{array}{l}\text { Depending on de- } \\
\text { mand retailer }\end{array}$ & Normal $\left(\mu=2^{\circ} \mathrm{C}, \sigma=0.5^{\circ} \mathrm{C}\right)$ \\
Transport DC-retailer & 0.125 & Normal $\left(\mu=2^{\circ} \mathrm{C}, \sigma=0.5^{\circ} \mathrm{C}\right)$ \\
Unloading at retailer & 0.031 & Normal $\left(\mu=15^{\circ} \mathrm{C}, \sigma=0.25^{\circ} \mathrm{C}\right)$ \\
Retailer & $\begin{array}{l}\text { Depending on de- } \\
\text { mand customer }\end{array}$ & Normal $\left(\mu=4^{\circ} \mathrm{C}, \sigma=0.5^{\circ} \mathrm{C}\right)$ \\
\hline
\end{tabular}

Values of other parameters for simulation are given in Table 2.4; e.g. selling price but also values of parameters of the microbiological growth model.

Table 2.4: Value of parameters based on Tromp et al. (2012), Broekmeulen \& van Donselaar (2009) and van Donselaar et al. (2006)

\begin{tabular}{ll}
\hline Parameter & Value \\
\hline Initial contamination & $\mu_{N}=2.95, \sigma_{N}=0.1 * \mu_{N}$ \\
$A$ & 2.95 \\
$C$ & $7.56-A$ \\
$\alpha_{B}$ & 0.104 \\
$\beta_{B}$ & 0.1573 \\
$\alpha_{M}$ & 14.525 \\
$\beta_{M}$ & -0.1365 \\
$\eta_{\text {waste }}$ & $5.3[\log \mathrm{cfu} / \mathrm{g}]$ \\
$\eta_{\text {unsafe }}$ & $6[\log \mathrm{cfu} / \mathrm{g}]$ \\
$z^{D C}$ & 1.96 \\
$e$ & 101 \\
$G$ & 4 \\
$f_{d}$ & {$[0.12,0.11,0.125,0.16,0.255,0.23,0]$} \\
$a$ & 0.4 \\
$p$ & $€ 2.98$ \\
$\gamma$ & 0.0003 \\
\hline
\end{tabular}

\subsubsection{Food quality analysis}

Food quality is important in dealing with perishable products. For meat products food quality is closely related to food safety, as micro-organisms will cause quality decay and safety hazards $\left(N_{t r} \geq \eta_{\text {unsafe }}\right)$. In Table 2.5 , the average microbiological count for products sold with FSL and DSL without discount is listed. We see that the difference in 
average microbiological count at the point of sales is small, and below $\eta_{\text {waste }}$. However, we see that for FSL a small amount of spoiled products $\left(N_{t r} \geq \eta_{\text {unsafe }}\right)$ is sold, where products sold with DSL are always safe.

Table 2.5: Microbiological count and percentage of spoiled products sold of experiment 1-10

\begin{tabular}{lllll}
\hline & \multicolumn{2}{c}{ Fixed shelf life } & \multicolumn{2}{c}{ Dynamic Shelf Life } \\
\hline \multirow{2}{*}{ Experiment } & $\begin{array}{l}\text { microbiological } \\
\text { count }(\log \mathrm{cfu} / \mathrm{g})\end{array}$ & spoiled products & $\begin{array}{l}\text { microbiological } \\
\text { count }(\log \mathrm{cfu} / \mathrm{g})\end{array}$ & spoiled products \\
\hline $1+2$ & 3.33 & $0.00 \%$ & 3.37 & $0.00 \%$ \\
$3+4$ & 3.32 & $0.00 \%$ & 3.33 & $0.00 \%$ \\
$5+6$ & 3.35 & $0.01 \%$ & 3.4 & $0.00 \%$ \\
$7+8$ & 3.37 & $0.00 \%$ & 3.38 & $0.00 \%$ \\
$9+10$ & 3.33 & $0.00 \%$ & 3.35 & $0.00 \%$ \\
\hline
\end{tabular}

\subsubsection{Effect of dynamic shelf life at retailer}

For experiment 1-18 an optimal replenishment policy is determined by optimizing the safety factor z for 100 runs of 1846 days of which 21 are regarded as warming up period. For experiments 19-32 the optimal replenishment policy is determined by optimizing the safety factor for each discount percentage $x$ for a two runs of 1846 days. The thus obtained optimal setting is evaluated at high accuracy of 100 runs. With this we obtain an accuracy that implies a standard deviation of about $3 \%$ of the mean profit for all optimal solutions. In Table 2.6 results for the optimal $z^{*}, S^{*}$, profit per week for the retailer and waste and shortages are given for each experiment (Exp.) of scenario 1 to 4 .

\section{Basic scenario}

In the basic scenario with FSL the optimal safety factor $z^{*}$ is 0.6 , resulting in an average $S^{*}$ level of 12. Profit obtained per week at the retailer is $€ 26.94$ and $1.87 \%$ of the products are wasted. Furthermore, shortage levels are $5.27 \%$. In comparing those results with a DSL we see increased $z^{*}$ and $S^{*}$ values, a higher profit and a reduction in waste and shortages. The results are in line with what we expected as shelf life setting is often rather conservative (Soethoudt et al., 2012). Actual shelf life might be longer than the FSL indicates. A DSL can show the actual shelf life of a product, which seems to be longer and therefore results in more time to sell the product. More selling time results directly in less waste as demand uncertainty is becoming less important. Products that are not sold on one day can be sold the next day. Less waste also reduces the amount of 
Table 2.6: Results of scenario 1-4

\begin{tabular}{lllllll}
\hline Scenario & Exp. & $z^{*}$ & $S^{*}$ & Profit & Waste & Shortages \\
\hline 1 & 1 & 0.6 & 12 & $€ 26.94$ & $1.87 \%$ & $5.27 \%$ \\
(Basic) & 2 & 1.3 & 14 & $€ 28.23$ & $1.33 \%$ & $1.87 \%$ \\
2 & 3 & 0.4 & 11 & $€ 15.67$ & $1.49 \%$ & $6.81 \%$ \\
Profit & 4 & 0.9 & 13 & $€ 16.72$ & $0.75 \%$ & $3.61 \%$ \\
margin) & 5 & 0.9 & 13 & $€ 42.02$ & $2.96 \%$ & $3.44 \%$ \\
& 6 & 1.6 & 15 & $€ 43.50$ & $1.84 \%$ & $1.22 \%$ \\
3 & 7 & 0.4 & 7 & $€ 14.76$ & $5.14 \%$ & $7.58 \%$ \\
(Demand) & 8 & 1 & 8 & $€ 16.31$ & $2.46 \%$ & $4.18 \%$ \\
& 9 & 1 & 20 & $€ 44.79$ & $1.14 \%$ & $2.57 \%$ \\
& 10 & 1.6 & 22 & $€ 45.94$ & $0.68 \%$ & $0.98 \%$ \\
4 & 11 & 0.1 & 10 & $€ 13.01$ & $20.61 \%$ & $7.16 \%$ \\
(Different & 12 & 0.5 & 12 & $€ 24.95$ & $4.05 \%$ & $7.81 \%$ \\
product) & 13 & 1.6 & 15 & $€ 28.88$ & $0.64 \%$ & $1.05 \%$ \\
& 14 & 2 & 16 & $€ 29.01$ & $0.65 \%$ & $0.56 \%$ \\
\hline
\end{tabular}

shortages. Although the model corrects for expected waste shortages will always occur due to uncertain demand.

\section{Effect on profit margin}

Products at a supermarket have different profit margins. Some products are used to attract consumers and therefore have a low profit margin where as other products are used to gain profit. Changing the profit margin influences profit and order-up-to levels and therefore the waste and shortage levels. Waste is increased compared to the basic scenario and shortages decreases with a higher profit margin. Out-of-stock (OOS) situations become more expensive and therefore safety factor is increased to prevent OOS. As a result of increasing $S^{*}$, more waste is obtained. The opposite results are obtained when profit margins are lower.

\section{Effect of demand}

The results of scenario 3, show that a decrease in demand reduces profit, not only because less products are sold but also because waste and shortages increase. With a lower demand, the safety factor decreases and vice versa for higher demand. This occurs for both FSL and DSL, although $z^{*}$ for DSL is always higher. When demand decreases, 
relative variance increases for Poisson demand, which causes the increase in waste and shortages. As waste reduces profit more than OOS situations it is optimal to reduce the safety factor.

\section{Effect of shelf life (different product)}

Scenario 4 tests the effect of shelf life. Scenario 1 included a shelf life of 8 days. In scenario 4, shelf life was reduced to 5 days for experiment 11 and 12 and increased to 10 days for experiment 13 and 14. A decreased shelf life decreases $z^{*}, S^{*}$ and profit while waste and shortages increases. Results show that a decreased shelf life makes it complicated to have the right amount of products in stock and sell them before they spoil compared to a longer shelf life. With a short shelf life products are moving too slowly in the chain resulting in high waste figures. After production products arrive 1 day later at the DC and it will take at least 1 day as well before the retailer receives the product. The DC is only delivered 3 times a week therefore products delivered at the retailer can be stocked at the DC for more than 1 day. When the shelf life is 5 days, the delay in moving products through the SC can result in the delivery of products with a low remaining shelf life at the retailer. This increases the amount of products wasted at the retailer as there is a limited time to sell them. For a longer shelf life it is easier to anticipate on the uncertainty in demand and the retailers' performance is improved. This implies that investing in shelf life extension might be worth to investigate for products with a short shelf life to improve SC performance. Furthermore benefits of DSL increase when shelf life decrease.

\subsubsection{Evaluation of DC ordering policy}

The DC is evaluated on shortages and waste. In Table 2.7 results of scenario 1 and 5 are given. In scenario 0, the DC orders three times a week on Monday, Wednesday and Friday. Shortages obtained are low for both FSL and DSL. Waste is not present in both experiments (1 and 2). When changing the number of ordering days for the DC to only twice a week (experiment 15 and 16) or to 6 days a week (experiment 17 and 18) we see that there are no significant changes in results. Waste levels at DC are obviously still zero, and shortages at the DC are hardly affected. The order strategy of the DC does influence the performance of the retailer. When a $\mathrm{DC}$ orders every working day $(\mathrm{R}=1)$, products arriving at the retailer will have a longer remaining shelf life. This reduces waste levels and therefore shortage levels. On the other hand, shortage and waste levels increase when the DC orders less frequently. 


\section{Discounting and Dynamic Shelf Life}

Table 2.7: Shortages and waste obtained at DC and retailer

\begin{tabular}{llllll}
\hline Scenario & Exp. & Shortage DC & Waste DC & Shortage retailer & Waste Retailer \\
\hline 1 & 1 & $0.03 \%$ & $0.00 \%$ & $5.30 \%$ & $1.87 \%$ \\
& 2 & $0.03 \%$ & $0.00 \%$ & $1.87 \%$ & $1.33 \%$ \\
5 & 15 & $0.02 \%$ & $0.00 \%$ & $10.65 \%$ & $2.10 \%$ \\
& 16 & $0.03 \%$ & $0.01 \%$ & $2.74 \%$ & $1.95 \%$ \\
& 17 & $0.04 \%$ & $0.00 \%$ & $3.48 \%$ & $1.36 \%$ \\
& 18 & $0.05 \%$ & $0.00 \%$ & $1.80 \%$ & $0.93 \%$ \\
\hline
\end{tabular}

\subsubsection{Effect of discount}

When incorporating discounting, two different factors are tested, first the moment of discounting, either at the last day of shelf life or one day earlier. Secondly, the influence of the picking order (initial LEFO-FEFO ratio) is evaluated. When a discount is applied we assumed that the percentage of consumers shifting from LEFO to FEFO purchase is equal to the discount given.

\section{Timing}

To test the effect of the moment of applying the discount, we compared applying a discount on the last day of shelf life with applying a discount one day earlier. The results shown in Figure 2.3 show that profit and shortage levels are affected by changing the moment of discounting. When discounts are applied two days instead of only on the last day, profits decrease and shortages are increased. Optimal order-up-to levels are slightly decreased when discounts are applied earlier. Waste levels are mainly affected by the discount percentage rather than the moment of discount.

\section{Picking order}

It is assumed that benefits of discounting products are influenced by the LEFO-FEFO ratio. Discounting attracts consumers to cheaper but less fresh products and discounting will have more of an effect when more consumers initially buy fresher products. Therefore, the effect of discounting is tested with different LEFO-FEFO ratios. As the previous results indicate that applying a discount only on the last day of shelf life is more attractive from a retailers perspective, this setting is used. Figure 2.4 shows the average optimal order-up-to level for a FSL and DSL of different LEFO ratios with the optimal $z^{*}$. For FSL the $S^{*}$ decreases or remains the same when more discount is given For DSL we see 

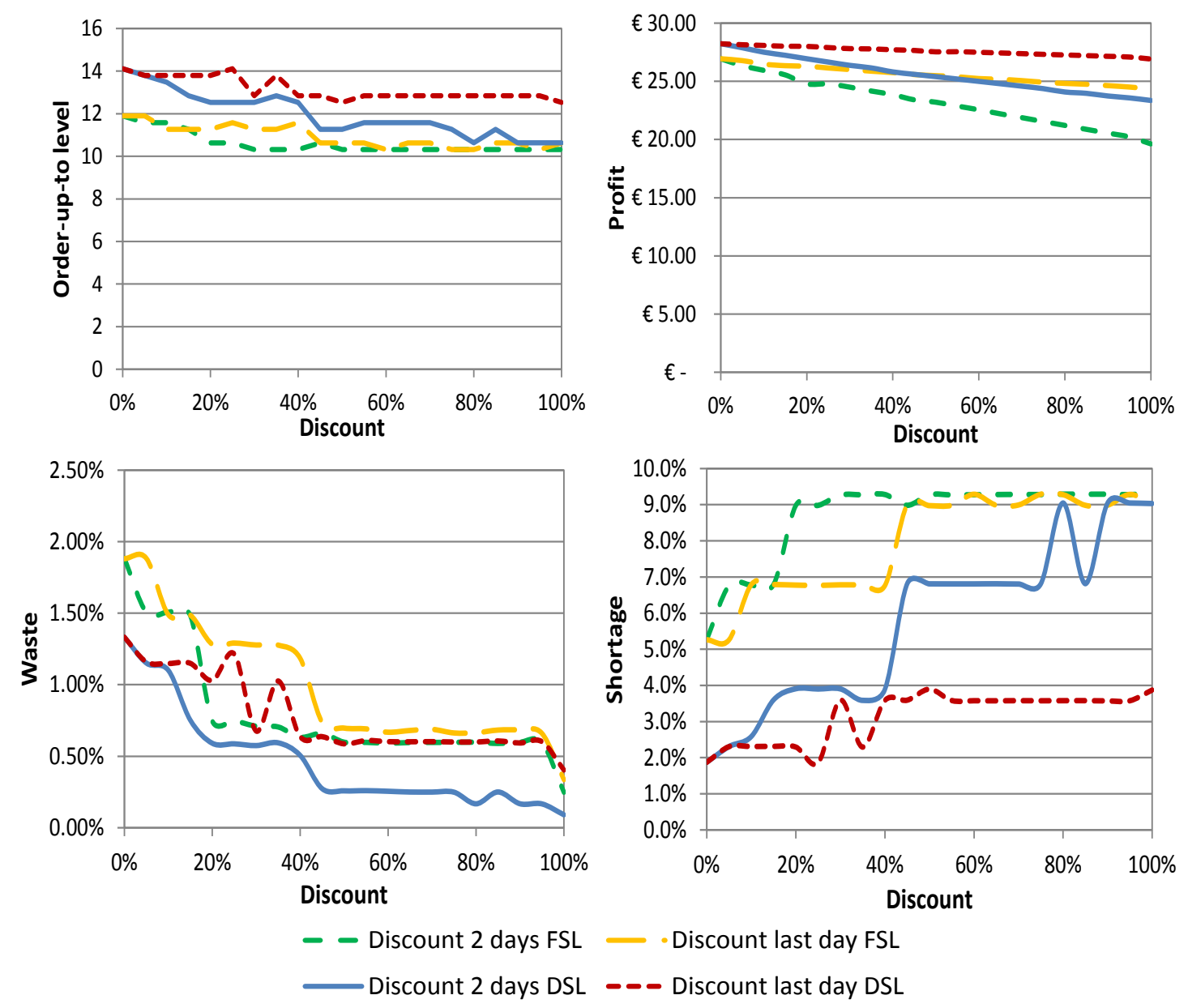

- Discount last day FSL

Discount last day DSL

Figure 2.3: Optimal ordering levels $\left(S^{*}\right)$, profit, waste and shortages for different moments of discount for FSL and DSL (last day of shelf life or day before).

that $S^{*}$ changes with different discount rates and becomes almost similar for all LEFO percentages. Furthermore we see that $S^{*}$ levels for FSL are lower than for DSL as we already concluded with previous results.

Figure 2.5 shows the profit, waste and shortages for FSL and DSL. Comparing profit levels (A and B) a clear distinction can be made between FSL and DSL. For FSL, profit is always decreasing for every LEFO-FEFO ratio where for DSL profit increases as long as there are initially more LEFO than FEFO consumers. When products of lower quality are sold as well, the retailer waste less products and $S^{*}$ can be increased. When there are more FEFO consumers than LEFO consumers, discounting decreases profit but it still reduces waste. In graph C and D the waste reduction for FSL and DSL at different discount percentages is shown. Both graphs show a similar decrease although initial waste levels are lower for DSL, which indicates that discounting is an effective strategy to reduce waste. Results show a somewhat irregular pattern. This is caused by the discretization of the order-up-to level, based on profit maximization, although the profit difference might 


\section{Discounting and Dynamic Shelf Life}

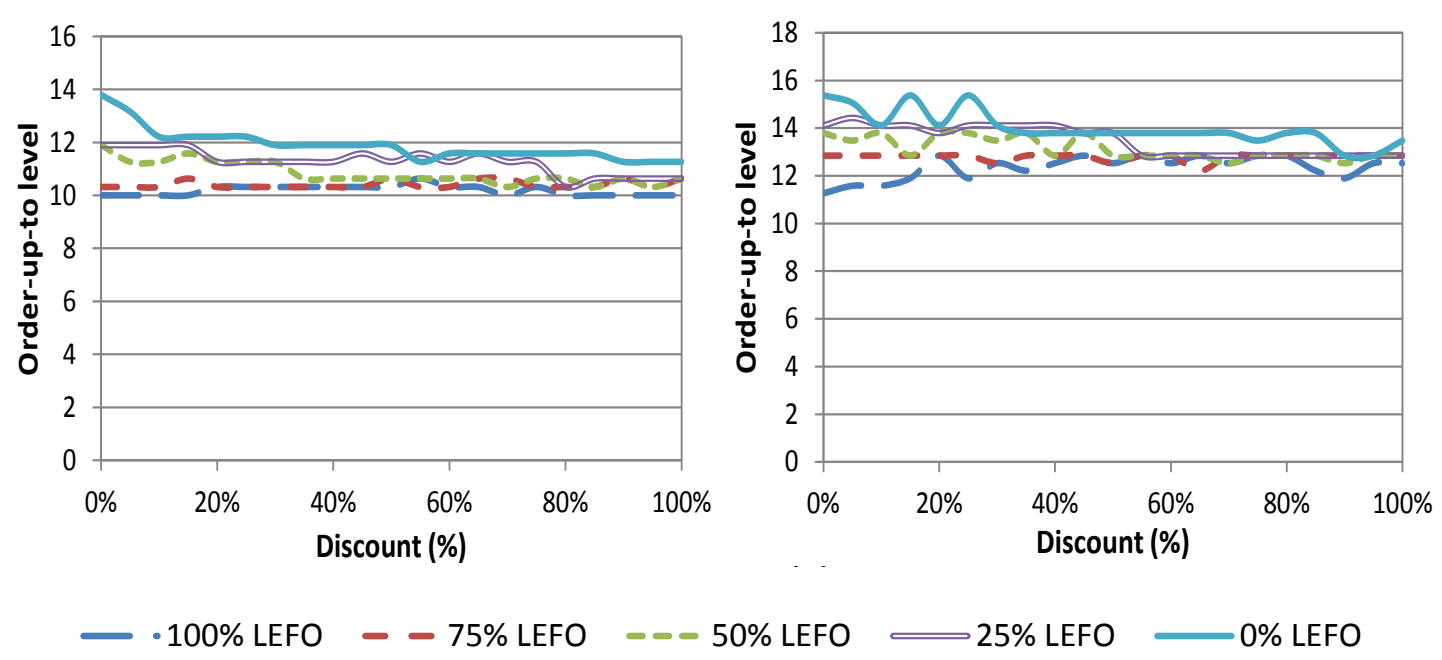

Figure 2.4: Optimal $S^{*}$ values for Fixed shelf life (left) and Dynamic shelf life (right)

be small. $S^{*}$ is rounded towards the nearest integer number, which can result in a change in $S^{*}$ where the initial difference in $z^{*}$ is small between two discount levels.

The last two graphs (E and F) in Figure 2.5 show the shortages. The results show that discounting increases shortage in some cases (DSL and LEFO\% <75\%) and that more LEFO consumers give higher shortage percentages. The increase in shortage over discount is caused by a lower $S^{*}$, which is beneficial for waste levels, but increases shortage levels. The decrease over decreased $\mathrm{LEFO} \%$ is in line with the higher waste percentages obtained at higher LEFO\% as waste and shortages are directly linked to each other. Ordering new products is based on current inventory levels. When a lot of products are wasted during period, the replenishment quantity might be too low and shortages occur in the next period. Expected waste is incorporated in the replenishment order however it is based on the average FEFO sales during a period. As demand is stochastic, there can be a variation between actual and expected sales which causes the shortages. Moreover, shortages obtained for FSL are higher than for DSL, which can also be explained by the higher waste levels for FSL. Concluding that DSL results on average in more time to sell products, variation in demand will have less influence for DSL and shortages are less likely to occur.

\subsection{Conclusion and discussion}

In this research we studied the effect of discounting and DSL on the replenishment of a retailer and on its performance in terms of waste, profit, shortages and product quality. Discount levels and replenishment quantities are optimized integrally. We studied the 

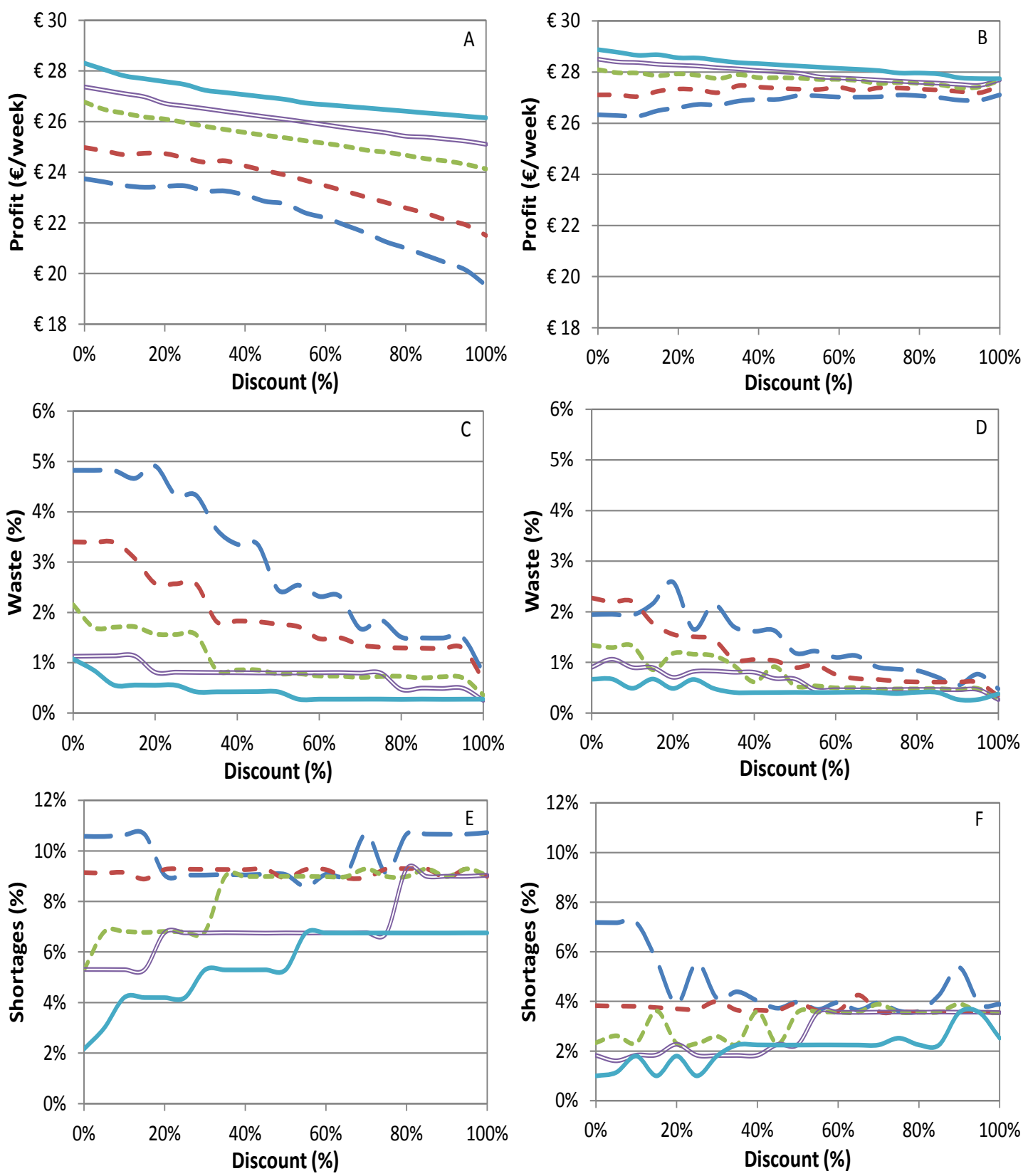

$-\cdot 100 \%$ LEFO $--75 \%$ LEFO $---50 \%$ LEFO $\longrightarrow 25 \%$ LEFO $-0 \%$ LEFO

Figure 2.5: A: Profit FSL, B: Profit DSL, C: Waste FSL, D: Waste DSL, E: Shortage FSL, F: Shortage DSL 


\section{Discounting and Dynamic Shelf Life}

effect of discounting and DSL separately and when combined. Both actions proved to be effective in reducing food waste, however applying DSL is more beneficial than applying a discount. The combination of both DSL and discounting proves to be the most effective strategy. Results show that, compared to a FSL, stock levels can be increased when a DSL applies. Furthermore, DSL results in less waste, more profit and less shortage compared to FSL. We also showed that DSL ensures food safety. When applying DSL the shelf life is based on the actual product quality and a retailer can be sure that he is selling safe products. Under DSL, products are wasted only if they are of (too) low quality; under FSL, also products of good quality might be wasted. Discounting reduced food waste with FSL and DSL however a profit increase is only obtained when discounting is combined with DSL.

For shelf life setting we used a limit of $\eta_{\text {waste }}=5.3 \mathrm{log} \mathrm{cfu} / \mathrm{g}$ is used a temperature of 5.5 ${ }^{\circ} \mathrm{C}$. This is safe on two sides, first the actual spoilage point will be at $\eta_{\text {unsafe }}=6 \mathrm{log} \mathrm{cfu} / \mathrm{g}$ (Tromp et al., 2012) and secondly the average temperature of the simulated SC is lower than $5.5^{\circ} \mathrm{C}$ so shelf life might be longer than the 8 days used for FSL. However, Table 2.5 shows that spoiled products are sold, even with those boundaries. When we relax those boundaries, e.g. by extending FSL from 8 to 9 days, even more products will be sold spoiled. Our research show that applying DSL increases profit, however this is without accounting for the costs of DSL implementation. Costs for DSL can be related to the TTI to show the quality of the product. Instead of including those costs, this study helps in assessing how much it may cost by comparing the difference in profits between DSL and FSL. For example in the base case the profit difference is $€ 1.29$, which relates to $€ 0.04$ per product. Another assumption we made related to DSL, is a perfect TTI. In real life indicators might have an error, which can affect food safety. This can be included in the model but is omitted as no data is available on the accuracy of a TTI. Unlike many studies (e.g. Farughi et al., 2014; Liu et al., 2008; Qin et al., 2014) we did include a shift in the LEFO-FEFO ratio instead of a demand increase when applying discount. We assumed the fraction of LEFO consumers that switch to FEFO corresponds to the discount level. When a larger percentage of consumers will shift, waste can be further reduced with higher profit levels and/or lower discount levels. An increased demand will most likely decrease waste levels and increase profit levels, for both FSL and DSL. In practice it can happen that that consumers substitute their initial purchases by the discounted meat, increasing the demand of that product, but decreasing the demand of the other product. With this, the overall meat demand will then remain equal. We see that consumer behaviour impacts the retailer performance. Therefore, it is recommended for further research to focus more on the in-store consumer behaviour and investigate topics such as the LEFO-FEFO ratio and substitution. The model we used here is specified to meat products, however by adjusting the quality model it can be applied for other fresh products. Our results show that discounting and a DSL are both effective strategies for reducing food waste by the 
retailer. Furthermore, this research highlights the importance of using DSL to both the retailer and the food producer. Another recommendation for producers would be for them to investigate shelf life extension as it would improve their performance as well. 


\section{Chapter 3}

\section{Product substitution to increase profit and reduce food waste at retailers}

Buisman, M. E., Haijema, R., \& Hendrix, E. M. T. (Submitted) Product substitution to increase profit and reduce food waste at retailers. 


\section{Abstract}

Substitution behaviour can have a significant influence on a retailer's profit and waste. This chapter investigates to what extent information on consumers' willingness to substitute can be used to improve the replenishment decisions, in terms of profit, waste and service levels. In this study, we compare two order policies: one policy neglects substitution and sets order quantities for products independently, and a new policy that sets order quantities for all products simultaneously and is anticipating product substitution. Both policies are analysed by simulation-based optimization. To facilitate a fast and accurate determination of parameter values, a heuristic search procedure is presented besides an exact enumerative approach.

Results show that the new policy may improve profit and reduce generation of waste in cases where consumers are willing to substitute products in case of out-of-stock situations. Moreover, it shows the trade-off between having a high service level, or high profit and low waste levels. Including substitution behaviour of consumers is of greater importance for cases in which products have a shorter shelf life. When, for a product the willingness of consumers to substitute is low, the product can be ordered independently of other products. On the contrary, if the willingness to substitute is very high, it may be optimal to take the product out of the assortment, although this might not be preferred by the retailer. 


\subsection{Introduction}

Food waste at retailers is both an economical and an environmental issue, and should thus be prevented (Papargyropoulou et al., 2014). In this chapter, we analyse an alternative replenishment policy that incorporates substitution behaviour of the consumer.

Retailers sell many different products in their shop and for each individual item, replenishment decisions have to be taken. These decisions can either be done manually or are supported by computer aided ordering $(\mathrm{CAO})$ or automated store ordering (ASO) systems. However, both approaches usually focus on individual products and therefore do not consider product substitution. At best, product substitution is anticipated informally in practice by setting different service levels for different products belonging to the same product category. Commonly used service levels are the non-stock-out probability and the fill rate. The impact of (differentiated) service levels on a retailer's profit and waste is not (a priori) known. As the main objective of a retailer is profit maximization, retailers are interested in a way to set replenishment quantities that maximise their profits.

Retailers want to serve consumers at any time of the day and thus have the tendency to hold high inventory levels for every product in practice. For non-perishable products this is acceptable, as unsold goods can be sold later on. However, for perishable products, this strategy will lead to high waste levels due to product spoilage. When retailers accept outof-stock situations for some products, and offer consumers at the same time a substitute, inventory levels can be lowered and waste levels can be decreased. Research shows that consumers do accept substitute products in out-of-stock situations, although customer satisfaction might decrease (Gruen et al., 2002). This chapter investigates how information on consumers' willingness to substitute can be used in improving the replenishment decisions.

The focus of this chapter is on improving the replenishment decisions for two perishable products that are partly substitutes in case of an out-of-stock situation. According to Van Woensel et al. (2007), the willingness to substitute is for highly perishable products larger than for non-perishable products. Reasons for consumers to consider substitution are an out-of-stock situation of the preferred product, or a better value-for-money of a substitute product.

Price based substitution (as in Gao et al., 2019) is not considered in this chapter, neither do we consider quality or age-based substitution (e.g., see Chew et al., 2014). It has been shown in previous research (e.g. Chen et al., 2015; Sachs, 2015; Hübner et al., 2016) that incorporating stock-out-based substitution in the replenishment decisions, can increase profit. However, it is not yet fully clear to what extend the trade-off between profit, waste and service levels are affected. In this chapter, we optimize and compare two policies and 
report profit, waste, and $\beta$-service levels. We consider the fill rate (or $\beta$-service level) to be an appropriate service level definition in this context, as it indicates the fraction of demand that should is lost or met by a substitute, which is more informative than the stock-out probability ( $\alpha$-service level). First, we optimize for each product independently a base stock policy using a single product model (that thus does not include product substitution). Next, we optimize simultaneously the replenishment parameters using a multi-product model that includes product substitution. By a multi-product simulation model, we compare both approaches and report profit, waste, and $\beta$-service levels.

The objective of this chapter is three-fold: (i) to present an approach to exploit product substitution in setting replenishment decisions, (ii) to generate managerial insights in the effect of product substitution on profit, waste and service levels, and (iii) to present a heuristic that facilitates the (heuristic) search for optimal replenishment parameters.

The remainder of this chapter is organized as follow. In Section 3.2 we discuss the relevant literature on inventory systems dealing with substitution and explain the contribution to the literature. Using a simulation optimization approach, which is presented in Section 3.3 and the heuristic explained in Section 3.4, 576 instances are solved and some managerial insights are discussed in Section 3.5. The chapter finishes with conclusions and a discussion in Section 3.6.

\subsection{Related literature}

This chapter focuses on the replenishment decision for multiple perishable products that are (partly) substitutes to each other. Most studies on the replenishment decision of multiple products that include product substitution are single period models or models for non-perishable products, see Section 3.2.1. The problem is hardly studied for perishable food products. In Section 3.2.2. We discuss papers that are closest related to this chapter. For a more general review on inventory management of perishable products we refer to review papers such as Karaesmen et al. (2011); Bakker et al. (2012) and Janssen et al. (2016).

\subsubsection{Substitution and replenishment decisions}

Many studies are available on inventory decisions, which include substitution (Shin et al., 2015). One of the first studies which include product substitution in inventory decisions is McGillivray \& Silver (1978), where the effect of substitution is analysed for a periodic review model. Classical inventory models, such as the EOQ model or the newsvendor model are extended by the incorporation of substitution (e.g. Drezner et al., 1995; Khouja 
et al., 1996; Smith \& Agrawal, 2000; Shah \& Avittathur, 2007). The inclusion of product substitution has shown to have a positive effect on the retailers profit (Shin et al., 2015). Moreover, for two product case, Transchel (2017) already showed that it may be profitable to store only one of the products, based on the substitution fractions and the product prices.

In several cases, inventory decisions are combined with pricing decisions, and thus often price based substitution is considered, instead of the out-of-stock substitution considered in our study (e.g. Akçay et al., 2010). Moreover, substitution is often incorporated in assortment planning. Assortment planning deals with the decisions which products to offer, but often exclude the decision on how much products to have in stock. However, the combination of assortment planning with shelf space allocation is increasing (Hübner \& Kuhn, 2012).

Besides, it is worth to mention that consumer driven substitution is not only studied in a retail context, but also considered in production planning decisions e.g. (Han et al., 2012; Zeppetella et al., 2017).

As our focus is on perishable products, we skip a detailed review of papers on nonperishable products. Instead, we refer to Shin et al. (2015).

\subsubsection{Replenishment including substitution for perishable products}

To our knowledge, only a few studies include substitution in the replenishment decisions for perishable products. In most studies, the replenishment decision is limited to a single period and/or a single product that is differentiated by the age of the products. The (single) replenishment decision relates to new items and demand and substitution is agebased. Downward substitution happens in case an older product is accepted when a product of a preferred age is out of stock (similar to LIFO issuance). Such models are mostly applied to blood products but can be generalized, e.g. to a retail setting.

An analytical solution for a two product case is found by Deniz et al. (2010), where the replenishment of a single product is optimized under age-based substitution. By assuming an immediate replenishment, i.e. excluding lead times, the problem becomes more tractable and an analytical approach is possible. For the case of a bakery, Sainathan (2013) optimizes the replenishment with a Markov decision process. Downward substitution, i.e. substitution between the old and the new product, is present. Besides the optimization of the replenishment quantities for the new product, decisions on pricing are made. Chen et al. (2015) also study a case where only downward substitution applies, however in their study, substitution is supplier-driven. In the study of Chew et al. (2014), replenishment and pricing decisions for multiple products are also included. Based on the 
inventory of the old product, order decisions are made for the new product, as well as the discount given on the old product.

Two studies are known which include empirical research, both Kök \& Fisher (2007) and Sachs (2015) study sales data of retailers to find the substitution ratios between the products. Kök \& Fisher (2007) uses these substitution fractions found to optimize both inventory and assortment decisions.

Next to substitution by age, substitution may also be based on other product attributes,

e.g. brand, flavour. Examples of studies for substitution of perishable products where the substitution is based on the product attribute can be found in the studies for blood products, as they are differentiated by blood type. Several studies can be found related to blood banks, for example Haijema et al. (2005); Duan \& Liao (2014); Najafi et al. (2017) and Dillon et al. (2017). However, as the retail setting differs from the context at hospitals and blood banks, we will not go into detail. Retailers do not have full control of the product withdrawal, as consumer pick products themselves, and may decide to not substitute, resulting in lost sales, which is not the case at the blood bank.

\subsubsection{Contribution to the literature}

To our knowledge, no study exists that considers the replenishment decisions for perishable products with substitution in a retail setting, where the products can be ordered individually instead of only order the new product. The known papers considering product substitution at the retail and include perishability incorporate substitution between old and new product, and thus only decide on order quantities for the new product. In the settings of blood banks, often substitution between old and new products is present. Furthermore, the blood bank itself can decide on how to use products as a substitute, where at the retail, the consumer decides on the substitution. Thus, our study extends the literature on inventory decisions for perishable products by considering a case for the retailer with two perishable products with a shelf life more than one period, with the inclusion of lead-time. Moreover, our study focusses on the trade-off between profit, waste and service levels, which is not present in the other studies.

\subsection{Method}

To analyse the effect of including substitution in replenishment decisions, a simulation based optimization model has been developed. The effect can be in terms of profit increase, waste decrease or obtaining better service levels. First the problem description is given, followed by the notation and the mathematical model. 


\subsubsection{Problem description}

We focus on a product category of a retailer that consists of $N$ different products with a fixed maximum shelf life $M_{i}$. We assume those products can (partly) serve as a substitute, and thus a fraction $\left(\gamma_{i j}\right)$ of the consumers consider product $j$ to be a substitute for product $i$. Only one-way substitution is included. The retailer faces a Poisson demand for all $N$ products meaning that consumers arrive at the rate of the Poisson distribution and request 1 item of the product. The retailer places an order at the beginning of the period, before opening hours. This order will arrive after closing, resulting in an effective lead-time of one day and an effective shelf life of $M_{i}-1$.

\subsubsection{Notation}

Sets and indices:

$i, j \in\{1, . ., N\} \quad$ Products

$t \in\{1, . ., T\} \quad$ Time periods

$r, m \in\left\{1, . ., M_{i}\right\} \quad$ Remaining shelf life

Parameters:

$S_{i} \quad$ Order-up-to level of product $i$

$\mu_{i} \quad$ Mean demand of product $i$

$\gamma_{i j} \quad$ Substitution fraction of product $i$ to product $j$

$p_{i} \quad$ Sales price of product $i$

$c_{i} \quad$ Cost of product $i$

a Fraction FIFO consumers

$M_{i} \quad$ Maximum shelf life of product $i$ 
Variables:

$I_{\text {tir }} \quad$ Inventory of product $i$ at the beginning of time period $t$ with remaining shelf life $r$

$D_{t i} \quad$ Initial demand of product $i$ during time period $t$

$E O_{t i} \quad$ Estimated outdating of product $i$ at time period $t$

$Q_{t i} \quad$ Order quantity of product $i$ at time period $t$

$X_{t i} \quad$ Remaining demand of product $i$ that should be fulfilled by a substitute at time period $t$

$Z_{\text {tir }} \quad$ Products sold of product $i$ at time period $t$ with remaining shelf life $r$ without substitution

$U_{t i r} \quad$ Products sold of product $i$ at time period $t$ with remaining shelf life $r$ due to substitution

$W_{t i} \quad$ Waste of product $i$ at the end of time period $t$

$\Pi_{t} \quad$ Total profit of time period $t$

$D_{t i}, X_{t i}, Z_{t i r}, U_{t i r}$ will be split into FIFO $\left(D F_{t i}, X F_{t i}, Z F_{t i r}, U F_{t i r}\right)$ and LIFO $\left(D L_{t i}, X L_{t i}, Z L_{t i r}, U L_{t i r}\right)$, see the model below.

\subsubsection{Discrete time simulation model}

At the beginning of every period, the retailer places an order $Q_{t i}$ for all $N$ products, see equation (3.1), based on the order-up-to level $\left(S_{i}\right)$, the current inventory for product $i$ and the estimated outdating during that period. Outdating is estimated by the difference between the average FIFO demand per day and the current inventory with a remaining shelf life of 1 day, equation (3.2). This approach is taken for practical reasons. However, it might lead to an overestimation of the outdating.

$$
\begin{array}{ll}
Q_{t i}=\left[S_{i}-\sum_{r=1}^{M_{i}-1} I_{t i r}+E O_{t i}\right]^{+} & \forall t \in\{1, . ., T\}, i \in\{1, . ., N\} \\
E O_{t i}=\left[I_{t i 1}-\left(\mu_{i} * a\right)\right]^{+} & \forall t \in\{1, . ., T\}, i \in\{1, . ., N\}
\end{array}
$$

Demand for each product follows a Poisson distribution with mean $\mu_{i}$, equation (3.3). At a retailer, there are usually consumers who prefer the fresher products, and some that take the first product in the shelf. Therefore we split the total demand for the products into a FIFO and LIFO demand equation (3.4) and equation (3.5), with $a$ being the fraction of demand following FIFO withdrawal.

$$
\begin{array}{lr}
D_{t i} \sim \operatorname{Poiss}\left(\mu_{i}\right) & \forall t \in\{1, . ., T\}, i \in\{1, . ., N\} \\
D F_{t i}=\operatorname{round}\left(a * D_{t i}\right) & \forall t \in\{1, . ., T\}, i \in\{1, . ., N\}
\end{array}
$$




$$
D L_{t i}=D_{t i}-D F_{t i} \quad \forall t \in\{1, . ., T\}, i \in\{1, . ., N\}
$$

The product withdrawal by consumers for both LIFO and FIFO demand is the minimum of the products in stock of a certain age $r$ and the remaining demand that is not satisfied yet. Without loss of generality, it is assumed that customers preferring the freshest product arrive first at the supermarket, and thus LIFO demand is fulfilled before the FIFO demand.

$$
\begin{gathered}
Z L_{t i r}=\min \left\{I_{t i r}, D L_{t i}-\sum_{m=r+1}^{M_{i}} Z L_{t i m}\right\} \\
\forall t \in\{1, . ., T\}, i \in\{1, . ., N\}, r \in\left\{1, . ., M_{i}-1\right\} \\
Z F_{t i r}=\min \left\{I_{t i r}-Z L_{t i r}, D F_{t i}-\sum_{m=1}^{r-1} Z F_{t i m}\right\} \\
\forall t \in\{1, . ., T\}, i \in\{1, . ., N\}, r \in\left\{1, . ., M_{i}-1\right\}
\end{gathered}
$$

When the demand is not met, substitution might take place. The number of consumers requesting a substitute product is an average fraction $\left(\gamma_{i j}\right)$ of the consumers facing a stock-out and given by equation (3.8), with $Z_{\text {tir }}$ being the total demand which is already met (equation (3.9))

$$
\begin{aligned}
& X_{t i} \sim \operatorname{Binom}\left(D_{t i}-\sum_{r=1}^{R-1} Z_{t i r}, \gamma_{i j}\right) \quad \forall t \in\{1, . ., T\}, i \in\{1, . ., N\} \\
& Z_{t i r}=Z L_{t i r}+Z F_{t i r} \quad \forall t \in\{1, . ., T\}, i \in\{1, . ., N\}, r \in\left\{1, . ., M_{i}-1\right\}
\end{aligned}
$$

Similar to the initial demand, the demand arising due to substitution is also divided into LIFO and FIFO demand:

$$
\begin{array}{ll}
X F_{t i}=\operatorname{round}\left(a * X_{t i}\right) & \forall t \in\{1, . ., T\}, i \in\{1, . ., N\} \\
X L_{t i}=X_{t i}-X F_{t i} & \forall t \in\{1, . ., T\}, i \in\{1, . ., N\}
\end{array}
$$

As it is assumed that stock-outs are more likely to happen at the end of a day, the demand occurring due to substitution takes place after the initial demand of the product is fulfilled, first by the LIFO withdrawal, followed by the FIFO withdrawal.

$$
\begin{gathered}
U L_{t i r}=\min \left\{I_{t i r}-Z_{t i r}, \sum_{j \neq i} X L_{t j}-\sum_{m=r+1}^{M_{i}} U L_{t i m}\right\} \\
\forall t \in\{1, . ., T\}, i \in\{1, . ., N\}, r \in\left\{1, . ., M_{i}-1\right\} \\
U F_{t i r}=\min \left\{I_{t i r}-Z_{t i r}-U L_{t i r}, \sum_{j \neq i} X F_{t j}-\sum_{m=1}^{r-1} U F_{t i m}\right\} \\
\forall t \in\{1, . ., T\}, i \in\{1, . ., N\}, r \in\left\{1, . ., M_{i}-1\right\}
\end{gathered}
$$


At the end of a period, the inventory is updated for the next period, the shelf life is reduced and outdating is registered, consumer withdrawal is subtracted and the products ordered at the beginning of the day will arrive, equation (3.14), with $U_{\text {tir }}$ being the total demand fulfilled by substitution (equation (3.15)). Note: the effective shelf life of a product is $M-1$. A lead-time of 1 day $(L=1)$ is applied for the retailer.

$$
\begin{aligned}
& I_{t+1, n, r-1}=\left\{\begin{array}{ll}
I_{t i r}-Z_{t i r}-U_{t i r} & , 2 \leq r<M_{i}-1 \\
Q_{t i} & , r=M_{i}
\end{array} \forall t \in\{1, . ., T\}, i \in\{1, . ., N\}\right. \\
& U_{t i r}=U L_{t i r}+U F_{t i r} \quad \forall t \in\{1, . ., T\}, i \in\{1, . ., N\}, r \in\left\{1, . ., M_{i}-1\right\}
\end{aligned}
$$

\subsubsection{Performance indicators}

To analyse the performance of a retailer, several key performance indicators are used. To determine the optimal order-up-to level $S$, total profit is maximized. Profit is calculated by subtracting procurement costs from the revenue made (equation 3.16) and reported as daily profit (equation 3.17). Fixed ordering costs and holding costs are neglected as perishable products at a retailer are usually replenished daily together with many others and therefore ordering and transportation costs are shared among all those products (Buisman et al., 2019a). Excessive inventory levels do not occur, as the shelf life is short.

$$
\begin{array}{ll}
\Pi_{t}=\sum_{i=1}^{N}\left(p_{i} *\left(\sum_{r=1}^{R-1} Z_{t i r}+U_{t i r}\right)-Q_{t i} * c_{i}\right) & \forall t \in\{1, . ., T\} \\
\Pi=\frac{\sum_{t=w+1}^{T} \Pi_{t}}{T-w} &
\end{array}
$$

Waste is calculated for every product per period of time by equation (3.18). For the final analysis, waste is represented as percentage of total ordered products, equation (3.19).

$$
\begin{aligned}
& W_{t i}=I_{t i 1}-Z_{t i 1}-U_{t i 1} \quad \forall t \in\{1, . ., T\}, i \in\{1, . ., N\} \\
& W=\frac{\sum_{t=w+1}^{T} \sum_{i=1}^{N} W_{t i}}{\sum_{t=w+1}^{T} \sum_{i=1}^{N} Q_{t i}}
\end{aligned}
$$

Moreover, service level measures are included. The fraction of demand that can be fulfilled from stock for the non-substitute product is measured by the $\beta$-service level. The $\beta_{i^{-}}$ service level represents the fraction of fulfilled demand for product $i$, equation (3.20). For 


\section{Replenishment decsisons under substitution}

the product which remaining demand is fulfilled by the other product, the $\beta_{j}$-service level measures the fraction of fulfilled demand for product $j$, either by product $j$, or product $i$, equation (3.21), with $j \neq i$. To specify by which products this demand is fulfilled, we included the $\beta_{j i}$ and $\beta_{j j}$-service level as well. The $\beta_{j i}$-service level is the fraction of demand for product $j$ fulfilled by product $i$, equation (3.22), and the $\beta_{j j}$-service level is the fraction of remaining demand for product $j$ fulfilled by product $j$, equation (3.23). Those fractions are estimated, per day, in the simulation as:

$$
\begin{array}{lr}
\beta_{i}=\frac{\sum_{t=w+1}^{T} \sum_{r=1}^{R-1} Z_{t i r}}{\sum_{t+w+1}^{T} D_{t i}} & \forall i \in\{1, . ., N\} \\
\beta_{j}=\frac{\sum_{t=w+1}^{T} \sum_{r=1}^{R-1} Z_{t j r}+U_{t i r}}{\sum_{t+w+1}^{T} D_{t j}} & \forall j \in\{1, . ., N\}, j \neq i \\
\beta_{j i}=\frac{\sum_{t=w+1}^{T} \sum_{r=1}^{R-1} U_{t i r}}{\sum_{t+w+1}^{T} X_{t j}} & \forall j \in\{1, . ., N\}, j \neq i \\
\beta_{j j}=\frac{\sum_{t=w+1}^{T} \sum_{r=1}^{R-1} Z_{t j r}}{\sum_{t+w+1}^{T} D_{t j}} & \forall j \in\{1, . ., N\}
\end{array}
$$

\subsubsection{Optimization approach}

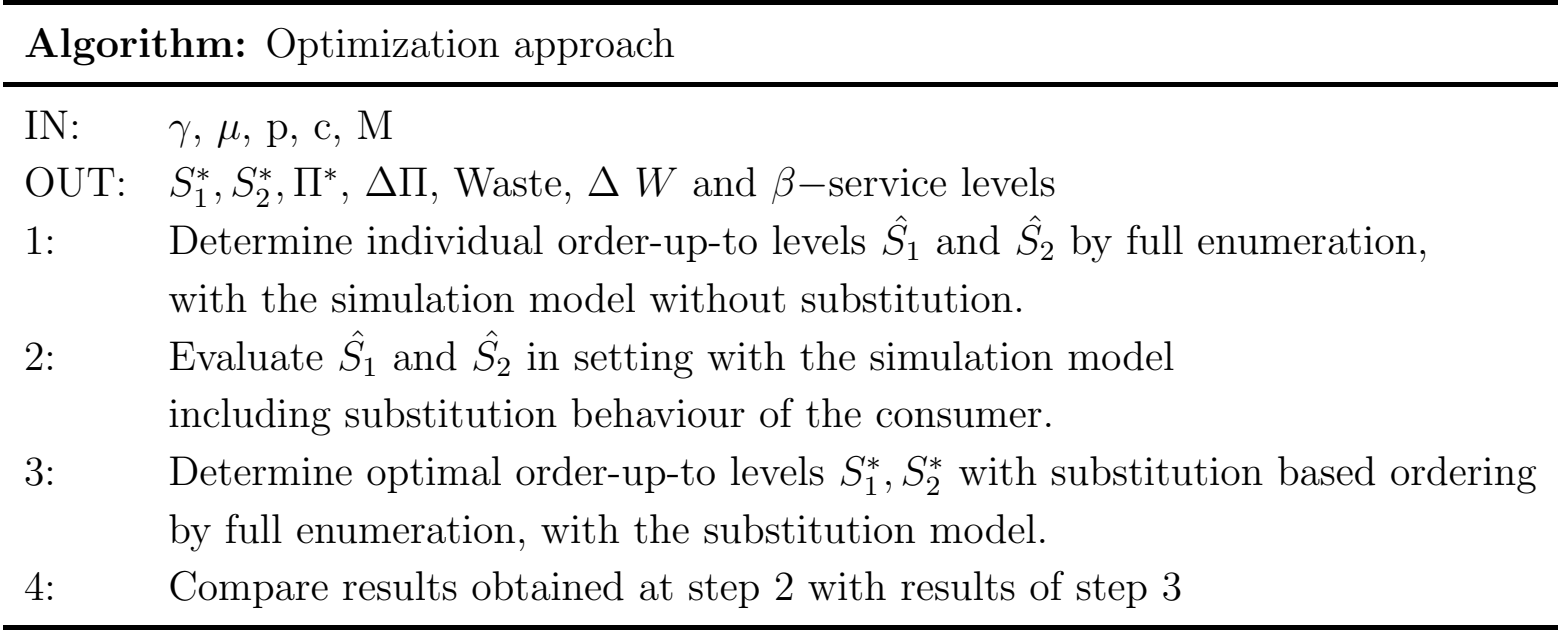

The optimization algorithm consists of multiple steps. First the optimal order-up-to level $S$ for a single product is determined with the help of the simulation model. This is done for each of the $N$ products individually, and thus substitution is not incorporated yet. The optimal order-up-to levels are determined based on profit maximization and their values are denoted $\hat{S}_{i}$. In the second step, the order-up-to levels $\hat{S}_{i}$ found in step 1 are used as 
reference values and therefore the simulation model is ran with these order-up-to levels when substitution does play a role. In the third step of the optimization, every order-upto level combination for the $N$ products is evaluated and the optimal order-up-to levels $S_{i}^{*}$ are determined. For the final analysis, the values obtained in step 2 are compared with the values obtained in step 3. Both in step 1 and in step 3 of the optimization a full enumeration is performed over a range of order-up-to levels $S\left(\left\{S_{i}^{\min }, \ldots, S_{i}^{\max }\right\}\right)$.

The lower and upper level $S_{i}^{\min }, S_{i}^{\max }$ of the search range are determined as follow. For Poisson demand, the order-up-to level $S$ can be calculated based on the lead-time $(L)$, review period $(R)$, the average demand $(\mu)$ and the safety factor $(z)$, using equation (3.24). In this research, the lead-time and review period are both fixed to 1 period.

$$
S=\mu_{R+L}+z * \sqrt{\sigma_{R+L}}
$$

To calculate the order-up-to level $S_{i}^{\max }$, the demand for both products is combined (thus, $\left.\mu=\mu_{1}+\mu_{2}\right)$ and the safety factor $(z)$ of 3 is used. A lower bound $\left(S_{i}^{\min }\right)$ of 0 is used as it might be beneficial not to have a product in stock at all, or to have a negative safety stock.

For the Base Case scenario, also a minimization on waste has been performed. The maximum waste reduction is determined without profit losses, compared to optimization without anticipating substitution. We also investigated a policy that considers the combined age distribution applying Stochastic Dynamic Programming (Hendrix et al., 2019). It appeared that the improvement over an order up to policy for our experiments is less than $1 \%$ for very perishable products and nearly absent when the shelf life is larger than 4. Therefore, we conclude that the easier to implement order-up-to policy is quite robust with respect to the optimal profit that can be reached.

\subsection{Heuristic to find optimal order-up-to levels with one-way substitution}

Optimizing replenishment for perishable products is a complex task, due to all the interdependencies between the products. Thus, the complete enumeration is computationally expensive. By developing a heuristic, the runtime needed to find the solutions decreases. The heuristic developed in this study still includes the simulation model described in section 3.3, and is therefore able to deal with a lead-time larger than zero and the perishability of the products. The heuristic is tested with two products and one-way substitution. The one-way substitution is included to get a clear view on how substitution affects the trade-off between profit, waste and service levels. Although the heuristic is only tested 
for a two-product case, an extension towards more products is possible. However, the managerial insights that will be obtained will not change much when more products are considered.

The heuristic is based on interesting characteristics of the results of Section 3.5, found by complete enumeration. For every experiment, the optimal $S_{1}^{*}$-level is higher than or equal to the optimal $\hat{S}_{1}$-level. For the product not serving as a substitute, the exact opposite result is obtained. For every experiment, the optimal $S_{2}^{*}$-level was equal to or lower than the optimal $\hat{S}_{2}$-level. These structural properties can be used to improve the optimization process, as many possible combinations of $S_{1}$ and $S_{2}$ will never be optimal. Thus, these combinations can be excluded.

The developed approach consists of several steps. In the first step, the individual order-upto levels $\hat{S}_{1}$ and $\hat{S}_{2}$ are calculated with full enumeration over all order-up-to levels $S$. The found order-up-to levels serve as starting point for the rest of the approach. Two starting points are used, (i) $S_{1}=\hat{S}_{1}$ and $S_{2}=\hat{S}_{2}$ (resulting in $\bar{\Pi}_{1}$ ) and (ii) $S_{1}=\hat{S}_{1}+\hat{S}_{2}$ and $S_{2}=0$ (resulting in $\bar{\Pi}_{2}$ ). Then, based on which of the two options results in the highest profit, the heuristic continues with another step. When $\bar{\Pi}_{1}>\bar{\Pi}_{2}$, the heuristic continues with step 3, otherwise it continues at step 4 . The third step consists of two parts. First we keep the total inventory level $\left(S_{1}+S_{2}\right)$ the same, but increase $S_{1}$ and decrease $S_{2}$ by 1 unit. This is iteratively done until no better solution is found for 3 consecutive runs. Then the neighbourhood of the best solution is checked, to see if a better solution exists, by either fixing $S_{1}$ or $S_{2}$ and in-/decreasing the other by 1 unit. When the best solution is found, and 3 consecutive runs do not give a better solution, the search stops. When step 4 is applied, $S_{2}$ is always equal to 0 , and $S_{1}$ is iteratively in-/decreased by 1 unit until no better solution is found (in terms of profit) for 3 consecutive runs. Then the optimal solution is found and the procedure stops.

\subsection{Numerical results}

In this section, we first discuss the experimental design, followed by the results. Besides discussing all results combined, an elaborate discussion on the Base Case results is presented. The evaluation of the Base Case is twofold, both on profit maximization and on waste minimization without profit losses.

\subsubsection{Experimental design}

The Base Case studied in this section is a setting where two highly perishable products $(N=2)$ are considered. We assume both products to have the same mean demand 
Heuristic Find optimal order-up-to levels

\begin{tabular}{|c|c|}
\hline IN: & $\gamma, \mu, \mathrm{p}, \mathrm{c}, \mathrm{M}$ \\
\hline OUT: & $S_{1}^{*}, S_{2}^{*}, \Pi^{*}$ (and Waste and $\beta$-service levels) \\
\hline 1: & Determine individual order-up-to levels $\hat{S}_{1}$ and $\hat{S}_{2}$ \\
\hline 2: & $\begin{array}{l}\text { Set } \hat{\Pi} \text { with } S_{1}=\hat{S}_{1} \text { and } S_{2}=\hat{S}_{2} \text { and determine } \Pi_{x} \text { with } S_{1}=\hat{S}_{1}+\hat{S}_{2} \\
\text { and } S_{2}=0 \text { and determine highest profit. If } \hat{\Pi} \geq \Pi_{x} \text { continue with step } 3 \text {, } \\
\text { else continue with step } 4\end{array}$ \\
\hline 3.1: & $\begin{array}{l}\text { Iteratively increase } S_{1}\left(S_{1}+1\right) \text { and decrease } S_{2}\left(S_{2}-1\right) \text { until best solution } \\
\text { is found in terms of profit. Update } S_{1} \text { and } S_{2}\end{array}$ \\
\hline 3.2: & $\begin{array}{l}\text { Iteratively check neighbourhood }\left\{\left(S_{1}+1, S_{2}\right) /\left(S_{1}-1, S_{2}\right) /\left(S_{1}, S_{2}+1\right) /\right. \\
\left.\left(S_{1}, S_{2}-1\right)\right\} \text { of best solution found so far until no better solution is found. } \\
\text { Update } S_{1} \text { and } S_{2} \text {. STOP }\end{array}$ \\
\hline 4: & $\begin{array}{l}\text { Iteratively in-/decrease } S_{1} \text { until no better solution is found. Update } S_{1} \text {, } \\
\text { keep } S_{2}=0 \text {. STOP }\end{array}$ \\
\hline
\end{tabular}

$\left(\mu_{1}=\mu_{2}=5\right)$, procurement costs $\left(p_{1}=p_{2}=€ 0.5\right)$ and shelf life $\left(M_{i}=3\right)$. Furthermore, we assume a selling price $\left(c_{i}\right)$ of $€ 1$ for both of the products, thus resulting in a profit margin of $50 \%$. The symmetry between the products gives a good understanding about the effect of substitution behaviour that will not be influenced by other parameters. For the base case, we investigate the setting of a mixture between FIFO and LIFO consumer withdrawal (50\% FIFO). To analyse the influence of asymmetric demand, asymmetric procurement costs, asymmetric shelf life and the FIFO-LIFO ratio, multiple experiments are performed. Furthermore, the effect of a longer shelf life is investigated. The complete overview of the experimental factor values induced is given in Table 3.1. The parameter values are listed in Table 3.2. A full factorial design is used, resulting in 576 experiments. The model is implemented in Matlab2018a. A run length of $T=10000$ periods is applied, of which $w=20$ are considered as warm-up period. To deal with the stochasticity arising from the demand and the binomial distribution for the substitution, all experiments are executed 20 times with different demand datasets and different a (fixed) seed for the binomial distribution. This resulted in a variety in profit levels of $1.29 \%$. Due to the discrete nature of the order-up-to levels $S$, the results listed below are for one specific setting.

\subsubsection{Overview: effect of substitution based ordering}

The average results per experimental factor are given in Table 3.3. For every factor, the number of experiments is given, the average profit and waste changes, compared to applying the individual order-up-to levels $\hat{S}_{i}$, as well as the corresponding $\beta$-service 
Table 3.1: Experimental values for the 576 experiments

\begin{tabular}{ll}
\hline Factor & Values \\
\hline$\gamma_{21}$ & $\in\{0.5,0.75,0.9,1\}$ \\
$\left(\mu_{1}, \mu_{2}\right)$ & $\in\{(5,5),(3,7),(7,3)\}$ \\
$\left(M_{1}, M_{2}\right)$ & $\in\{(3,3),(5,5),(3,5),(5,3)\}$ \\
$\left(p_{1}, p_{2}\right)$ & $\in\{(0.5,0.5),(0.7,0.7),(0.5,0.7),(0.7,0.5)\}$ \\
$a$ & $\in\{0,0.5,1\}$ \\
\hline
\end{tabular}

Table 3.2: Parameter values

\begin{tabular}{ll}
\hline Parameter & Value \\
\hline$T$ & 10000 periods \\
$N$ & 2 products \\
$c_{i}$ & $€ 1$ \\
\hline
\end{tabular}

levels.

The following tendency can be observed using individual order-up-to levels $\hat{S}_{i}$ compared to substitution based order-up-to levels $S_{i}^{*}$. When many consumers are willing to buy a substitute, it becomes more beneficial to have higher stock levels of the product that serves as substitute (e.g. product 1) and less of the other product (product 2). When basically everyone is accepting a substitute, the largest profit increases will be obtained when only product 1 is in stock compared to keep both product 1 and 2 in stock. Furthermore, waste levels can be reduced a lot. As all consumers accept a substitute, demand for both products can be combined which reduces the relative variation. This facilitates a better determination of the optimal order-up-to levels $S$ and leads to an increase in profit and decrease in waste. When less consumers are willing to buy a substitute, the optimal order-up-to levels $S^{*}$ will be lower for $S_{1}$ and higher for $S_{2}$. Due to consumers that are not willing to buy a substitute, it is necessary to keep both products in stock to maintain the sales. Otherwise, the amount of lost sales will be too high and profit decreases. The waste reduction therefore decreases, as both products have to be kept in stock, although substitution based ordering still improves compared to independent ordering.

The $\beta_{1}$ - and $\beta_{2}$-service levels are for every case high and thus most consumers will leave the store with a product. The $\beta_{22}$-service level shows which fraction of the demand for product 2 is also fulfilled by product 2 , where the $\beta_{21}$-service level indicates which fraction of the demand is fulfilled by product 1 . When the level of substitution is high, and thus $S_{2}$ is low in many cases, demand for product 2 is fulfilled by product 1 , reducing the 
Table 3.3: Average results of all experiments and datasets listed per experimental factor. Number of experiments per dataset $(\#)$, relative change in profit $\left(\Delta \Pi=\frac{\Pi^{*}-\hat{\Pi}}{\hat{\Pi}}\right)$ and waste $\left(\Delta W=\frac{W^{*}-\hat{W}}{\hat{W}}\right)$ compared to suboptimal replenishment $(\hat{S})$ and $\beta$-service levels for the optimal order-up-to levels $S^{*}$.

\begin{tabular}{l|ccccccc}
\hline Dataset & $\#$ & $\Delta \Pi$ & $\Delta \mathrm{W}$ & $\beta_{1}$ & $\beta_{2}$ & $\beta_{22}$ & $\beta_{21}$ \\
\hline All Experiments & 576 & $8.89 \%$ & $-35.27 \%$ & $94.99 \%$ & $89.38 \%$ & $55.55 \%$ & $93.70 \%$ \\
$\gamma_{21}=0.5$ & 144 & $4.53 \%$ & $-22.16 \%$ & $93.56 \%$ & $87.56 \%$ & $76.48 \%$ & $96.83 \%$ \\
$\gamma_{21}=0.75$ & 144 & $9.33 \%$ & $-37.91 \%$ & $95.35 \%$ & $89.09 \%$ & $53.02 \%$ & $93.11 \%$ \\
$\gamma_{21}=0.9$ & 144 & $9.34 \%$ & $-37.91 \%$ & $95.35 \%$ & $89.09 \%$ & $53.02 \%$ & $93.10 \%$ \\
$\gamma_{21}=1$ & 144 & $12.37 \%$ & $-43.09 \%$ & $95.72 \%$ & $91.77 \%$ & $39.71 \%$ & $91.77 \%$ \\
$\left(\mu_{1}, \mu_{2}\right)=(5,5)$ & 192 & $9.34 \%$ & $-35.52 \%$ & $95.42 \%$ & $89.77 \%$ & $58.26 \%$ & $94.22 \%$ \\
$\left(\mu_{1}, \mu_{2}\right)=(3,7)$ & 192 & $10.21 \%$ & $-26.68 \%$ & $93.24 \%$ & $91.71 \%$ & $67.92 \%$ & $95.57 \%$ \\
$\left(\mu_{1}, \mu_{2}\right)=(7,3)$ & 192 & $7.13 \%$ & $-43.60 \%$ & $96.32 \%$ & $86.66 \%$ & $40.49 \%$ & $91.31 \%$ \\
$\left(\mathrm{M}_{1}, \mathrm{M}_{2}\right)=(3,3)$ & 144 & $7.29 \%$ & $-17.72 \%$ & $95.41 \%$ & $84.69 \%$ & $46.12 \%$ & $90.62 \%$ \\
$\left(\mathrm{M}_{1}, \mathrm{M}_{2}\right)=(5,3)$ & 144 & $7.90 \%$ & $-66.60 \%$ & $97.33 \%$ & $88.91 \%$ & $44.33 \%$ & $94.82 \%$ \\
$\left(\mathrm{M}_{1}, \mathrm{M}_{2}\right)=(3,5)$ & 144 & $4.13 \%$ & $-2.42 \%$ & $90.55 \%$ & $90.96 \%$ & $71.69 \%$ & $93.60 \%$ \\
$\left(\mathrm{M}_{1}, \mathrm{M}_{2}\right)=(5,5)$ & 144 & $16.25 \%$ & $-54.33 \%$ & $96.69 \%$ & $92.95 \%$ & $60.08 \%$ & $95.77 \%$ \\
$\left(p_{1}, p_{2}\right)=(0.5,0.5)$ & 144 & $5.67 \%$ & $-40.36 \%$ & $96.08 \%$ & $93.11 \%$ & $65.16 \%$ & $95.45 \%$ \\
$\left(p_{1}, p_{2}\right)=(0.7,0.5)$ & 144 & $3.21 \%$ & $-25.32 \%$ & $91.39 \%$ & $93.87 \%$ & $84.19 \%$ & $95.48 \%$ \\
$\left(p_{1}, p_{2}\right)=(0.5,0.7)$ & 144 & $20.74 \%$ & $-45.55 \%$ & $98.47 \%$ & $82.58 \%$ & $19.67 \%$ & $92.20 \%$ \\
$\left(p_{1}, p_{2}\right)=(0.7,0.7)$ & 144 & $5.94 \%$ & $-29.84 \%$ & $94.03 \%$ & $87.95 \%$ & $53.20 \%$ & $91.68 \%$ \\
$a=0$ & 192 & $15.75 \%$ & $-33.80 \%$ & $91.04 \%$ & $82.66 \%$ & $60.10 \%$ & $86.79 \%$ \\
$a=1$ & 192 & $4.81 \%$ & $-40.25 \%$ & $97.24 \%$ & $94.41 \%$ & $57.77 \%$ & $98.07 \%$ \\
$a=0.5$ & 192 & $6.11 \%$ & $-31.75 \%$ & $96.71 \%$ & $91.06 \%$ & $48.79 \%$ & $96.24 \%$ \\
\hline
\end{tabular}

$\beta_{22}$-service level. Moreover, the $\beta_{21}$-service level increases with lower substitution rate $\gamma_{21}$. Thus, a higher percentage of consumers that want to buy a substitute will find a product in the shelf.

When the demand of the two products is not equal, and the product not serving as a substitute has a higher demand than the substitute, a larger profit increase is obtained when substitution based ordering is applied. However, a larger waste reduction is obtained when the substitute product has the highest average demand. In absolute figures, waste is lower for a higher shelf life. As the optimal order-up-to levels $S$ change, a change in the $\beta$-service level is obtained.

The effect of substitution is more profound when the shelf life of the products is short. With a maximum shelf life of 3 days, the obtained profit increase is larger compared to a maximum shelf life of 5 days. Although, a larger waste reduction is obtained for the 
case of a longer shelf life. Service levels are also higher for a longer shelf life, as it is less complicated to keep the right number of products in stock. This can also be seen in the results of a different shelf life for both products. When the shelf life of product 1 is lower than of product 2, hardy any profit increase or waste reduction is obtained. As a longer shelf life allows to have a higher quantity in stock, product 2 should be still available. When the shelf life of product 2 is lower than of product 1 , there is a large reduction in waste levels possible. The reduction in the $\beta_{22}$-service level indicates the reduced inventory level of the product.

Interesting results are obtained when the ratio between procurement cost and sales price differs. The best profit increase is found when product 2 is less profitable than the substitute (product 1). In this case, it is beneficial to keep mainly the substitute in stock, as consumers will buy a more profitable product in the end. The high stock level of $S_{1}$ also results in a high $\beta_{1}$-service level. When the substitute product is the least profitable product, it is beneficial to avoid out-of-stock situations of the non-substitute product and thus keep both products in stock. Therefore, the possible profit increase is small. When both products have a similar but high procurement cost, it becomes more expensive to have waste products. Therefore both products will have a reduced optimal order-up-to level $S$ and thus a reduction in $\beta$-service levels is present. Although, anticipating on substitution still results in a profit increase.

With a full FIFO withdrawal, the highest waste reductions can be obtained. As a FIFO consumer withdrawal of the products is the most efficient in terms of waste, which leads to the potential improvements. On the other hand, the profit increases are not very high. With a full LIFO withdrawal, high profit increases can be obtained by the combined replenishment strategy. The mixture of a $50 \%$ FIFO and a $50 \%$ LIFO withdrawal shows results most similar to the full FIFO withdrawal.

\subsubsection{Trade-off between service levels and profit or waste}

In retail, it might be interesting to set target service levels for the products, to satisfy consumers as much as possible. In the previous sections we showed that, due to substitution effects, it could be more attractive to have only the product serving as substitute in stock instead of both products. A decision to keep also product 2 in stock provides a trade-off between profit and service level, and between waste and the service level. Figure 3.1 depicts this trade-off. When the service level of product $2\left(\beta_{22}\right)$ becomes higher, the maximum daily profit reduces. Note, these profit levels are present when the optimal order-up-to level $S_{1}$ is used in combination with the preferred order-up-to level $S_{2} \in\{0, \ldots, 22\}$. In addition, high service levels cause high waste levels. 


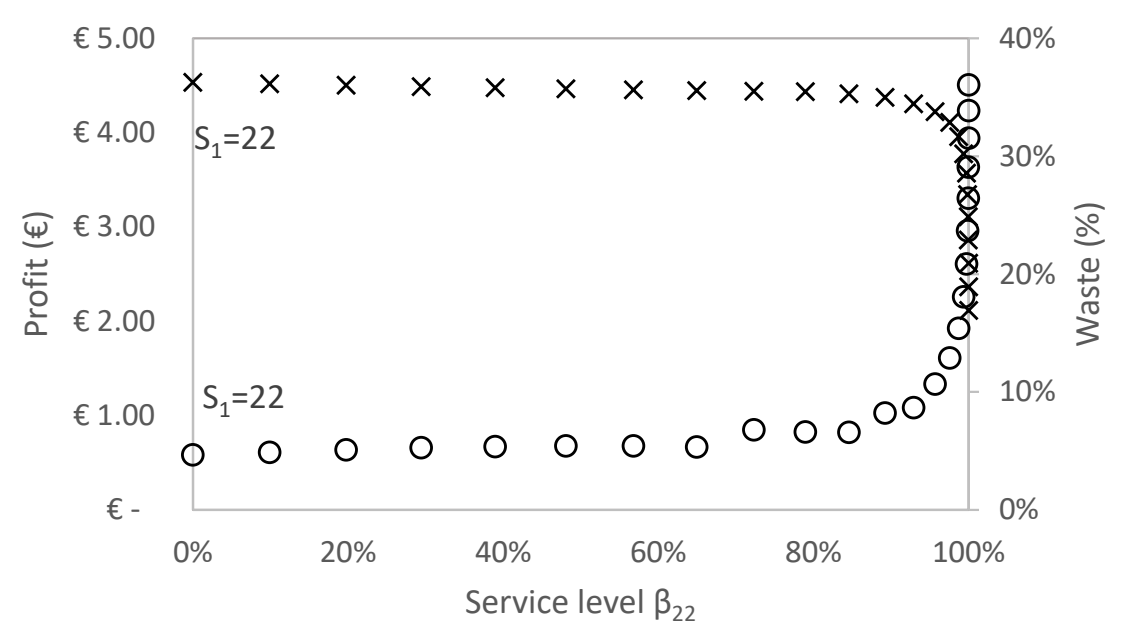

Figure 3.1: Total profit in $€(\times)$ and Waste in $\%$ (०) vs. service levels $\beta_{22}$ for $S_{2} \in\{0, \ldots, 22\}$ with full substitution $\left(\gamma_{21}=1\right)$

Figure 3.1 illustrates the conflicting character of service level and profit objectives. On the one hand, the retailer might want to serve the customers always with the product consumers demand, where on the other hand they aim for high profit levels or low waste levels. Figure 3.1 shows that in case of full substitution, high profit levels and low waste levels are obtained when the service level of product $2\left(\beta_{22}\right)$ is not too high. Although, when a retailer is willing to compromise slightly on the service level (e.g. $\beta_{22}=80 \%$ ), decent profits can be made together with low waste levels.

\subsubsection{Maximizing profit and minimizing waste for the Base Case}

In section 3.5.2, the focus of optimization was on profit maximization. Although a waste reduction was obtained for every experiment, larger waste reductions might be possible with different order-up-to levels $S$. In this section, we therefore analyse the Base Case both on profit maximization and on waste minimization. When the focus of the optimization is on waste minimization, a profit constraint is added, to ensure profit does not decrease compared to the profit values of the individual ordering settings. Moreover, the more detailed results also give extra insight into the trade-off between service-levels and profit or waste. The experimental settings of the Base Case are as follows, the average demand $\mu_{1}=\mu_{2}=5$, the procurement costs $p_{1}=p_{2}=€ 0.5$ and the maximum shelf life $M_{i}=3$ for both products. Consumer withdrawal is considered a mixture between $50 \%$ FIFO and $50 \%$ LIFO.

Table 3.4 shows the optimal order-up-to levels $S^{*}$, the obtained profit and resulting waste (in percentages of total ordered quantity) and the various $\beta$-service levels are shown for the varying substitution behaviour $\gamma_{21}$. In these results the order-up-to levels $S^{*}$ 
Table 3.4: $S_{1}^{*}, S_{2}^{*}$, Profit $(\Pi)$, Waste $(W), \beta$-service levels and relative change in profit $\left(\Delta \Pi=\frac{\Pi^{*}-\hat{\Pi}}{\hat{\Pi}}\right)$ and waste $\left(\Delta W=\frac{W^{*}-\hat{W}}{\hat{W}}\right)$ compared to suboptimal replenishment $(\hat{S}) . \hat{S}_{1}=$ $\hat{S}_{2}=12$

\begin{tabular}{l|cccccccccc}
\hline$\gamma_{21}$ & $S_{1}^{*}$ & $S_{2}^{*}$ & $\Pi$ & $\Delta \Pi$ & $W$ & $\Delta W$ & $\beta_{1}$ & $\beta_{2}$ & $\beta_{22}$ & $\beta_{21}$ \\
\hline 0.5 & 13 & 10 & $€ 4.33$ & $1.29 \%$ & $7.11 \%$ & $-19.73 \%$ & $95.40 \%$ & $91.69 \%$ & $84.58 \%$ & $97.52 \%$ \\
0.75 & 15 & 7 & $€ 4.44$ & $3.04 \%$ & $5.39 \%$ & $-37.98 \%$ & $97.46 \%$ & $90.13 \%$ & $64.94 \%$ & $91.91 \%$ \\
0.9 & 15 & 7 & $€ 4.44$ & $3.04 \%$ & $5.39 \%$ & $-37.98 \%$ & $97.46 \%$ & $90.13 \%$ & $64.94 \%$ & $91.91 \%$ \\
1 & 22 & 0 & $€ 4.53$ & $5.28 \%$ & $4.68 \%$ & $-46.16 \%$ & $99.84 \%$ & $90.26 \%$ & $0.00 \%$ & $90.26 \%$ \\
\hline
\end{tabular}

Table 3.5: $S_{1}^{*}, S_{2}^{*}$, Profit $(\Pi)$, Waste $(W), \beta$-service levels and relative change in profit $\left(\Delta \Pi=\frac{\Pi^{*}-\hat{\Pi}}{\hat{\Pi}}\right)$ and waste $\left(\Delta W=\frac{W^{*}-\hat{W}}{\hat{W}}\right)$ compared to suboptimal replenishment $(\hat{S})$. Highest possible waste reduction without profit loss. $\hat{S}_{1}=\hat{S}_{2}=12$

\begin{tabular}{l|cccccccccc}
\hline$\gamma_{21}$ & $S_{1}^{*}$ & $S_{2}^{*}$ & $\Pi$ & $\Delta \Pi$ & $W$ & $\Delta W$ & $\beta_{1}$ & $\beta_{2}$ & $\beta_{22}$ & $\beta_{21}$ \\
\hline 0.5 & 11 & 9 & $€ 4.21$ & $1.12 \%$ & $3.55 \%$ & $-59.89 \%$ & $88.58 \%$ & $85.76 \%$ & $78.95 \%$ & $94.04 \%$ \\
0.75 & 19 & 1 & $€ 4.32$ & $0.39 \%$ & $2.82 \%$ & $-67.55 \%$ & $99.42 \%$ & $78.15 \%$ & $89.34 \%$ & $9.90 \%$ \\
0.9 & 19 & 1 & $€ 4.32$ & $0.39 \%$ & $2.82 \%$ & $-67.55 \%$ & $99.42 \%$ & $78.15 \%$ & $89.34 \%$ & $9.90 \%$ \\
1 & 19 & 0 & $€ 4.32$ & $0.39 \%$ & $1.49 \%$ & $-82.81 \%$ & $99.16 \%$ & $75.71 \%$ & $75.71 \%$ & $0.00 \%$ \\
\hline
\end{tabular}

for substitution based ordering. Furthermore, the in-/decrease in profit and waste is given. The differences are calculated by comparing order-up-to levels $S_{i}^{*}$ (step 3 of the optimization procedure) and individual order-up-to levels $\hat{S}_{1}$ (step 2 of the optimization procedure).

The results clearly have the same trend as shown in Table 3.3, when the willingness of consumers to substitute $(\gamma)$ increases, larger profit improvements are obtained. When all consumers are willing to substitute, it is the most profitable to have only product 1 in stock and divert all customers towards product 1 . This results in a high $\beta_{1^{-}}$and $\beta_{2}$-service level, however the $\beta_{22}$-service level is $0.0 \%$, as product 2 is not available. At lower substitution rates $\gamma$, it becomes more beneficial to have also product 2 in stock, this increases the $\beta_{22}$-service level but also increases the waste and decreases the profit level.

Table 3.5 show the optimal order-up-to levels $S$ when waste is minimized, without decreasing the profit levels obtained with $\hat{S}$. The combined order-up-to levels $S^{*}$ are lower for waste minimization than for profit maximization. This reduces the number of products that are sold, thus the obtained profit is lower. Although the profit is reduced, profit levels for waste minimization are still higher than the profit levels obtained at individual order- 
Table 3.6: Performance of heuristic. Per step the average number of runs needed and the number of experiments using this step, the relative change in profit $\left(\Delta \Pi=\frac{\Pi^{*}-\hat{\Pi}}{\hat{\Pi}}\right)($ in $\%)$.

\begin{tabular}{lccc}
\hline Step & \# runs & $\Delta \Pi$ & \# experiments \\
\hline 1 & 62 & - & 576 \\
2 & 2 & - & 576 \\
3.1 & 4.01 & $+1.57 \%$ & 432 \\
3.2 & 19.17 & $+5.76 \%$ & 432 \\
4 & 7.30 & $+12.98 \%$ & 144 \\
All & 98.48 & $+6.77 \%$ & 576 \\
\hline
\end{tabular}

ing. Moreover, the reduction of products in stock also reduces the number of products turning into waste at the end of their shelf life. Waste levels become significantly lower (e.g. $5.1 \%$ versus $1.8 \%$ for $\gamma_{21}=1$ ). Furthermore, the reduction in optimal order-up-to levels also decreases the service levels.

\subsubsection{Performance of the heuristic}

The developed heuristic is used for all 576 experiments listed in Section 3.5. In all cases, it led to the optimal solution found by full enumeration for both order-up-to levels $S$. At the second step of the procedure, a decision is made whether to continue with step 3 or step 4. In $75 \% \bar{\Pi}_{1} \geq \bar{\Pi}_{2}$ holds and thus step 3 is used. For all other cases $(25 \%)$ the heuristic continues at step 4 .

Table 3.6 shows the most improvement is obtained in the execution of the fourth step. As found in Section 3.5 it is sometimes optimal to have only product 1 in stock. This occurs mostly when substitution rates are high. Thus, the optimal solution will be found by step 4 in the heuristic. In the case where substitution rates are lower, the optimal solution for $S_{1}^{*}$ and $S_{2}^{*}$ will be much closer to $\hat{S}_{1}$ and $\hat{S}_{2}$ and therefore the best solution will be found by step 3 of the heuristic. As the optimal solution is close to $\hat{S}_{1}$ and $\hat{S}_{2}$, the profit increase obtained is also lower.

The advantage of using the heuristic versus the full enumeration is the saving in number of simulation runs. When full enumeration is used to obtain the optimal order-up-to levels $S_{i}^{*}$, the simulation model is ran for 1023 times. First $31+31=62$ times to determine $\hat{S}_{1}$ and $\hat{S}_{2}$, followed by $31 * 31=961$ times to determine the optimal order-up-to levels $S_{i}^{*}$. The number of runs needed by the heuristic to find the optimal solution is significantly lower, as shown in Table 3.6. In step 1 and 2 of the heuristic, still 64 runs are needed, but the number of runs needed to obtain optimal order-up-to levels $S_{i}^{*}$, in step 3 or 4 is reduced 
heavily. On average a $90.8 \%$ saving in the number of simulation runs is obtained.

\subsection{Conclusion and discussion}

In this chapter we optimised the replenishment for a retailer selling multiple, perishable products, where in case of out-of-stock one of the products can serve as a substitute. Replenishment decisions for multi-product inventory systems of perishables are under studied, especially the effect of product substitution on these decisions is hardly explored. We optimize the replenishment decisions by simulation-based optimisation. Results show the importance of a further investigation on substitution within inventory management as the improvements that can be made are significant, both in profit and waste levels.

When the willingness of consumers to substitute is high, it can be beneficial to only have one of the products in stock. Furthermore we show by simultaneously optimising the order-up-to levels $S$ and meanwhile considering substitution, that in any case it is possible to increase the profit and decrease product waste. The benefits of combining the products in the replenishment decisions become larger when the shelf life of the products is small, or when the two products have a different shelf life. Moreover, when the profit margin of the products differ, anticipating substitution becomes interesting when consumers are willing to substitute towards the product with a higher profit margin. Consumers in a supermarket will have different purchasing behaviours (i.e. FIFO or LIFO purchase). For all combinations, the incorporation of the substitution behaviour of consumers shows to have an effect on the retailer performance.

The approach also provides insight in what service level maximises profit. A too high service level will decrease the profit level and results in high product waste. A profit maximising retailer thus has to accept occasionally out-of-stock situations. In case of two products where product 1 is a substitute for the other, the availability of product 1 is key. For product 2 it is subtle, if consumers are willing to substitute, it may be optimal to offer product 2 but at a much lower availability or to ban product 2 from the assortment. This maybe strategically not desired as consumer satisfaction may decrease (too much) and profit increases will not be present when consumer decide to shop at another retailer. Trade-off curves show how a service level constraint on product 2 affect profit and waste.

In this research, we studied one-way substitution for a two-product case. In future research the model developed can be extended to more products and a longer shelf life. However, the search for optimal parameter values will take more time. Our search procedure has shown to be efficient. For the 576 experiments in this study, it does find the optimal replenishment parameters. Nevertheless, the procedure is a heuristic, as optimality can 
not be guaranteed for all other cases. The heuristic reveals great improvements in terms of numerical efficiency compared to complete enumeration. The set-up of the heuristic facilitates a fast and accurate optimization. The extension towards two-way substitution requires a different solution approach. Netessine \& Rudi (2003) already shows that the inclusion of two-way substitution does not guarantee the profit function to be unimodal in every case. In our study, the profit function shows a clear optimum, due to the one-way substitution.

This research indicates that products for which the willingness to substitute is low can be managed individually, whereas products that are easily be substituted may be better taken out of the assortment to reduce waste and to increase profit. 


\section{Chapter 4}

\section{Inventory optimization under consumer driven substitution for vertically differentiated products}




\section{Abstract}

In this research, assortment and inventory decisions for vertically differentiated products are simultaneously optimized. In the case a product is unavailable, either due to a stockout, or if it is not in the assortment, consumers will purchase a substitute product. In this study we include multiple substitution rounds. To find (near) optimal solutions for this complex problem, a heuristic is developed. This heuristic solves the case of $N$ products by iteratively aggregating to two products and decomposing successively.

The results of this study show the effect of combining the assortment and inventory decisions. A significant profit increase is obtained when these decisions are made simultaneously, mainly obtained by the increase of products in the assortment. Due to the consideration of substitution behaviour of the consumer, the variation in demand can be spread out over more products, thus reducing the risk of overstocking. The optimal inventory levels therefore increase, compared to the newsvendor quantities for individual products. By considering multiple substitution rounds, profit and inventory levels increase, compared to a single substitution round. Moreover, the profit margins, the distribution of the quality attribute of the product and the critical ratio of the newsvendor problem have an effect on the optimal assortment and inventory decisions. 


\subsection{Introduction}

The problem of managing inventory considering consumer-driven substitution is daily practice for retailers. Selecting the right set of products in the assortment, and determining the number of items to have in stock is a huge challenge for retailers. Moreover, the demand of consumers does depend on the set of available products and is thus influenced by substitution. Hence, it is important to gain insight in how substitution affects the optimal assortment and inventory levels and develop an efficient method to find solutions that maximize profit.

In this research, we consider inventory decisions for multiple, vertically differentiated products with consumer-driven substitution. Vertically differentiated products are products, which differ in price, but also in the quality attribute of the product. These could be for example a product of an A-brand, a similar product of a B-brand and an organic variant of the product. Substitution is present in many different forms. In our research, we consider consumer-driven, or consumer-initiated substitution, where the consumer voluntarily chooses a substitute (Shin et al., 2015). Substitution can also be firm driven, in this scenario the firm decides to on the substitute (Zeppetella et al., 2017). For consumer-driven substitution, two types can be distinguished. Dynamic/stock-out based substitution and static/assortment based substitution. Stock-out based substitution happens when a consumer does not find their preferred product but an empty shelf instead, and then decides to purchase a substitute (Mahajan \& van Ryzin, 2001). Assortment based substitution does not take into account the inventory levels, in the sense that a product is either in the assortment or not. When a consumer does not find their favourite product to be in the assortment of the store, they switch to another product which is carried in the assortment (Kök et al., 2008). Besides stock-out and assortment based substitution, there is substitution driven by product price (Shin et al., 2015). This occurs when consumers switch products based on prices; however, their initially preferred product is still available. In this research, we consider both assortment and stock-out based substitution. Products can be unavailable due to a stock-out, or a product does not belong to the optimal assortment carried by the store, and thus assortment-based substitution occurs.

Research has shown that consumers are often willing to buy a substitute from the same product category when their most preferred product is not available (Gruen et al., 2002; Van Woensel et al., 2007). For a retailer it is therefore critical to consider the product category as a whole when planning inventories and fully consider the substitution behaviour across the products, instead of optimising each product individually. Assortment planning considers product categories; however, in making the decision on inventory levels, i.e. how many products should be in stock, individual products should be considered. The decision on how many products to stock can affect the assortment carried by the store, 
as it could be optimal to not stock a product, which would be included in the optimal assortment, or the other way around (Gaur \& Honhon, 2006; Transchel, 2017). We study therefore inventory decisions for a retailer by considering both the product assortment and the stock levels of products.

Taking inventory decisions for multiple products together comes with challenges. By including substitution, the inventory decisions of the different products become interdependent, which increases the problem complexity. In this research, we present a heuristic, which iteratively solves a two-product case until the optimal stock levels are found for each product. Another challenge arises with the determination of the substitution fractions. Many studies included exogenously determined substitution fractions (e.g. Smith \& Agrawal, 2000; Netessine \& Rudi, 2003; Tan \& Karabati, 2013; Wu et al., 2018), but in this study, a utility-based choice model for consumer demand is used. With this utility model, the substitution fractions for all products can be calculated, based on the available assortment. Moreover, in our study, an unlimited amount of substitution rounds of consumers is considered. When a consumer does not find their preferred product, they substitute to 'neighbouring' products, i.e. substituting either upward or downward. When the number of products that are unavailable increase, the substitution fractions change. The substitution fractions thus depend on the assortment and inventory levels. Our research provides a method to cope with these changing substitution fractions. The use of multiple substitution rounds is hardly considered in the literature; mostly an approximation is used (e.g. Kök \& Fisher, 2007; Hübner et al., 2016).

Our problem has similarities to the problem considered by Pan \& Honhon (2012). However, in their study they only optimize assortment and do not consider inventory levels. As previous research (Transchel, 2017) indicates the importance of the joint optimization of assortment and inventory, we compare our results with the results obtained with the algorithm developed by Pan \& Honhon (2012).

The contribution of our study is three-fold: (i) we show the difference between pure assortment optimization and considering inventory levels in the decision of which products to include in the assortment, (ii) we provide a fast heuristic for a multi-product problem for vertically differentiated products and (iii) we show the importance of considering multiple substitution rounds. Moreover, this study provides insight in how demand uncertainty and substitution influences the inventory decisions.

Outline of the chapter is as follow, In Section 4.2, we discuss the literature that is most closely related to our research. The model is given in Section 4.3 followed by the solution method in Section 4.4. The numerical results are given in Section 4.5. The chapter finishes with the conclusion and some discussion in Section 4.6. 


\section{$4.2 \quad$ Literature}

Our study contributes to two streams of the Operations Management (OM) literature. Firstly, it contributes the literature on multi-product inventory planning with substitution, and, secondly, it contributes to the literature on assortment planning. There is a significant body of literature available on inventory management with product substitution. For an extensive overview of the literature, both on assortment and inventory planning, we refer to Shin et al. (2015). In remainder of this section, we first discuss shortly the most common demand models used followed by a description of studies including both inventory and assortment decisions but either include assortment or stock-out based substitution. Later on, studies are discussed that both include assortment and stockout based substitution. The section finishes with the few available studies that include multiple substitution rounds.

\subsubsection{Demand modelling}

The most popular demand models applied in the literature including consumer-driven substitution are consumer choice models or exogenous demand models (Hübner \& Kuhn, 2012). Choice models are often used for assortment planning (e.g. Mahajan \& van Ryzin, 2001; Cachon et al., 2005; Hopp \& Xu, 2005), however, to a lesser extent, present within research on inventory decisions (e.g. Shao et al., 2013; Honhon \& Seshadri, 2013; Wan et al., 2018). These choice models, for example multinomial logit or locational choice models, give the demand for specific products within a group of products based on consumer preferences and product parameters.

The use of exogenous demand functions, which give the demand for each product directly, is more common in studies on inventory decisions (e.g. Netessine \& Rudi, 2003; Tan \& Karabati, 2013), however also applied in the studies regarding assortment decisions (e.g. Ernst \& Kouvelis, 1999; Rajaram \& Tang, 2001).

\subsubsection{Inventory and assortment decisions}

The mentioned studies above consider either inventory/replenishment decisions or assortment planning decisions. Research that combine inventory and assortment decisions under consumer driven substitution is still limited, but increasing (Shin et al., 2015). As far as known, the study of Chand et al. (1994) is one of the first to optimize jointly the assortment and inventory. By cost minimisation, the number of parts needed by a manufacturer is optimized under one-way substitution and a fixed demand. A MINLP solves 
the problem initially, but the study shows the possibility of approximating the solution with a dynamic programming algorithm. Under a consumer choice model, van Ryzin \& Mahajan (1999) optimize both the assortment and the inventory levels for horizontally differentiated products (e.g. products with a different colour or flavour). The consumers' choice depend on the available assortment, and if this product of choice is not available, the consumer does not take a second substitution attempt. Li (2009) considers a similar problem; they allow unequal cost parameters, and include a variable demand. For a continuous store traffic an exact solution is found, when the store traffic is based on a discrete random variable, a profit rate heuristic is developed to find the optimal inventory levels of the products in the optimal assortment.

Besides assortment-based substitution, there is stock-out based substitution. Smith \& Agrawal (2000) jointly optimize the assortment and inventory levels for a retail store assuming the overall customer demand follows a negative binomial distribution. They include exogenous substitution fractions, given by substitution matrices, furthermore, only one substitution attempt is allowed. They use an approximation approach and include service levels for the individual products. With their study, they show the importance of including substitution effects when optimising the inventory levels.

\subsubsection{Single substitution round}

The previously mentioned studies either include assortment based or stock-out based substitution, where, if both the assortment as the inventory levels are optimized, both types of substitution do play a role. Our study considers both types of substitution. In line with the few studies available, which also both include assortment based and stock-out based substitution, we assume substitution fractions to be the same for both out-of-assortment (OOA) and out-of-stock (OOS) based substitution.

Gaur \& Honhon (2006) solve an inventory problem with stock-out based substitution for horizontally differentiated products (e.g. products that differ in colour or flavour), by using the results of the same problem with only OOA based substitution as a lower bound. For both cases a heuristic is developed. A locational choice model gives demand under substitution. Besides optimising the assortment and inventory levels for a retailer, Kök \& Fisher (2007) also develop a method to determine substitution fractions from sales data. Moreover, they propose an iterative heuristic, which considers shelf space resulting in a realistic assortment problem for a retailer selling multiple products. Hübner et al. (2016) solve a similar problem; however develop a faster and more accurate heuristic to solve

the problem. In a later study, Hübner \& Schaal (2017), solve an assortment-planning problem with shelf space constraints. A sequential solution approach solves the problem. First the assortment is determined and based on that assortment they find the optimal 
stock quantities. This approach allows for solving large instances of the problem.

\subsubsection{Multiple substitution rounds}

In almost all studies, only single substitution attempts are incorporated. In some studies the assumption is made that an increased substitution fraction for a single attempt is approximating the problem with multiple substitution attempts (Kok, 2003), however there are many studies where a single substitution attempt is used for convenience (e.g. Hübner et al., 2016). In our study, substitution fractions are determined based on the choice model and this choice model also provides the substitution fractions when multiple products are not available. This is indeed an increased fraction, compared to the first substitution attempt, however it is hard to determine exactly how much larger this fraction is without calculating it. Therefore, the approach we take gives more robust substitution fractions.

Only a few studies are available, which include multiple substitution attempts. Vaagen et al. (2009) considers a multi-item newsboy problem, under stock-out based substitution. A two-stage stochastic programming model is formulated to include the substitution attempts, although they are not able to capture the dependency between the offered products completely. A dynamic programming model is used in Honhon et al. (2010) for solving the assortment problem including the inventory levels, under discrete demand. Substitution occurs when a product is OOS, and is predetermined (i.e. a consumer has a first preference for product 3 , product 4 would be his second choice and the third preference is product number 1). Yücel et al. (2011) provide a mixed-integer programming model to find the optimal assortment and the corresponding inventory levels under consumer driven substitution. Both OOA and OOS based substitution are included. They show the impact of shelf space restrictions, ordering quotas and which suppliers to order at for this joint problem.

Considering the literature available on joint inventory and assortment optimization, our study has several contributions. First, we consider multiple substitution attempts and provide a procedure to calculate the individual substitution probabilities based on the available assortment. Secondly, by considering assortment and inventory decisions, we contribute to the small number of studies available in the literature. Thirdly, we provide a fast heuristic, to find (near) optimal solutions for product set of vertically differentiated products. 


\subsection{Model formulation}

To study the multi-item inventory problem for vertically differentiated products, a model is formulated. This model provides optimal inventory and assortment decisions, under consumer driven substitution. First, the notations used are listed, followed by the general model description, the modelling of the consumer demand and the substitution process.

\subsubsection{Notations}

Throughout the chapter, the following notations are used:

Parameters:

$i, j \quad$ Product index

$\mathcal{N} \quad$ Optimal assortment

$\mathcal{N}^{+} \quad$ Products still available from the optimal assortment $\mathcal{N}$

$\mathcal{N}^{-} \quad$ Products out-of-stock from the optimal assortment $\mathcal{N}$

$D_{i} \quad$ Initial demand for product $i \in\{1, \ldots, \mathcal{N}\}$

$D_{i}^{e} \quad$ Effective demand for product $i \in\{1, . ., \mathcal{N}\}$

$\gamma_{i j} \quad$ Fraction of consumers substituting from $i$ to $j$

$r_{i} \quad$ revenue of product $i \in\{1, \ldots, \mathcal{N}\}$

$c_{i} \quad$ costs of product $i \in\{1, . ., \mathcal{N}\}$

$v_{i} \quad$ Quality attribute of product $i \in\{1, \ldots, \mathcal{N}\}$

$Q_{i} \quad$ Inventory level of product $i \in\{1, . ., \mathcal{N}\}$

$\Theta \quad$ Random quality valuation of customer with the support $[0, \bar{\theta})$

$\Phi(\theta), \phi(\theta) \quad$ cdf and pdf of $\Theta$

$\Psi \quad$ Total market size (random variable)

\subsubsection{General problem description}

Consider a retailer selling $N$, partly substitutable products indexed by $i \in \mathcal{N}=$ $\{1, \ldots, N\}$. The products are vertically differentiated i.e. the products have different quality valuations, e.g. organic and non-organic products. Let $v_{i}$ denote the quality, $r_{i}$ the selling price and $c_{i}$ the costs of product $i$ respectively. Without loss of generality we assume $v_{1}<v_{2}<\ldots<v_{N}$ and $r_{i}>c_{i}>0$. For this single-period setting, the retailer needs to decide on the order quantities $Q_{i}$ for each product $i \in \mathcal{N}$ without knowing the uncertain product demand and not knowing the exact substitution fractions. The effective demand of product $i, D_{i}^{e}$ consist of (i) the primary demand $D_{i}$ (i.e. consumers who 
have product $i$ as their first choice) and (ii) the demand rising from substitution due to stock outs of products. A more elaborate explanation on the demand model is given in Section 4.3.3 and 4.3.4. Generally described, the retailer decides on the order quantities for all products such that following expected profit $(\Pi)$ is maximized

$$
\max \Pi=\sum_{i \in \mathcal{N}}\left(r_{i} \mathbb{E}\left[\min \left\{Q_{i}, D_{i}^{e}\right\}\right]-c_{i} Q_{i}\right)
$$

\subsubsection{Consumer choice and demand formulation}

We consider a random market size $D$ with known distribution. All consumers in that market are heterogeneous with respect to the perceived quality valuation $\left(\Theta v_{i}\right)$. This heterogeneity is captured by a random variable $\Theta$ whose distribution is assumed to be known. Let $\Phi(\cdot)$ and $\phi(\cdot)$ denote the cdf and pdf of $\Theta$ with the support $[\underline{\theta}, \bar{\theta}]$. For simplicity, let $\Phi(\cdot)=1-\Phi(\cdot)$. The second component affecting the consumer choice is the selling price, $r_{i}$, as such the net utility from product $i$ of a random consumer is

$$
U_{i}=\Theta v_{i}-r_{i}
$$

This choice model is a pure characteristics demand model, which is widely used to model demand for vertically differentiated products (Berry \& Pakes, 2007; Akçay et al., 2010). We consider that consumers choose among the set of available products, i.e., all products that are offered and that are still in stock. This induces that the purchasing probabilities depend on the available set of products. Let $\mathcal{N}^{+} \subseteq \mathcal{N}$ denote the set of products that are available (in stock) and let $\mathcal{N}^{-} \subseteq \mathcal{N}$ denote the set of products that are not available. If the entire set of products is still available, i.e., $\mathcal{N}^{+}=\mathcal{N}$, then the probability that a consumer chooses product $i_{x} \in \mathcal{N}^{+}$is denoted as $P_{i_{x}}\left(\mathcal{N}^{+}\right)$. Thus, products $i_{x} \in \mathcal{N}^{+}$with a negative $U_{i}$ are not purchased. Let $\mathcal{S}^{+}=\left\{i_{1}, i_{2}, \ldots, i_{m}\right\} \subseteq \mathcal{N}^{+}$with $\left\{i_{1}<i_{2}<\ldots<i_{m}\right\}$ denote the subset of products $i_{x}$ whose purchasing probability is strictly positive, i.e., $P_{i_{x}}\left(\mathcal{N}^{+}\right)>0$ and let $\mathcal{S}^{-}=\left\{j_{1}, j_{2}, \ldots, j_{p}\right\}$ with $\mathcal{S}^{-}=\mathcal{N}^{+} \backslash \mathcal{S}^{+}$denote the subset of products $j_{y}$ whose purchasing probability is zero, i.e., $P_{j_{y}}\left(\mathcal{N}^{+}\right)=0$. For notational convenience, we denote the "no purchase" option as the fictitious product $i=0$ with $r_{0}=v_{0}=c_{0}=0$. An example of the purchasing probabilities is given in Figure 4.1. For $N=3$ this shows the ranges of $\theta$ in which product 1,2 , and 3 are purchased or the consumer does not purchase a product, which then provides the purchasing probabilities $P_{i}\left(\mathcal{N}^{+}\right)$, based on the intersections between the utility lines. 


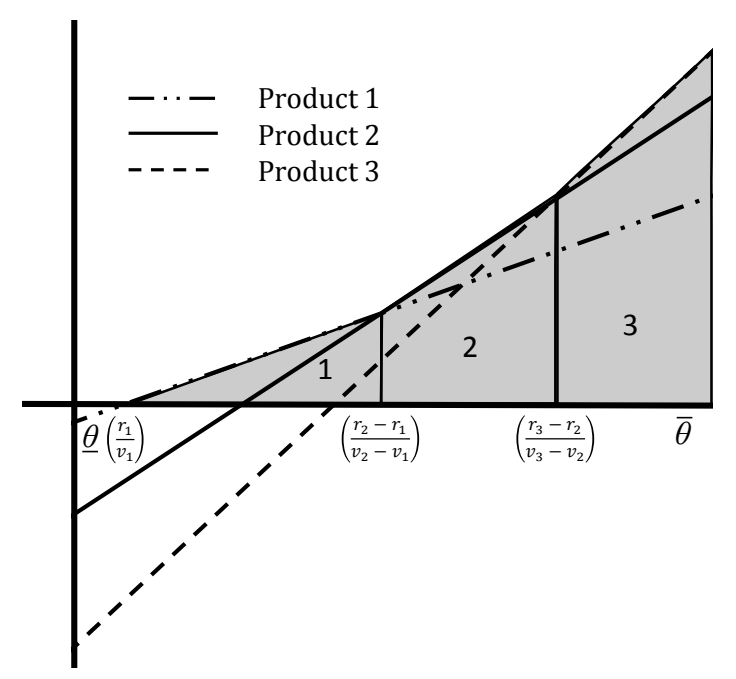

Figure 4.1: Fractions of consumers that would purchase a product, given all products are available.

Pan \& Honhon (2012) showed that a necessary and sufficient condition for all products in $\mathcal{S}^{+}$is $\underline{\theta} \leq \frac{r_{i_{1}}}{v_{i_{1}}}<\frac{r_{i_{2}}-r_{i_{1}}}{v_{i_{2}}-v_{i_{1}}}<\ldots<\frac{r_{i_{m}}-r_{i_{m-1}}}{v_{i_{m}}-v_{i_{m-1}}}<\bar{\theta}$ which implies that the product-specific purchasing probabilities are strictly positive as such

$$
P_{i_{x}}\left(\mathcal{N}^{+}\right)=\Phi\left(\frac{r_{i_{x+1}}-r_{i_{x}}}{v_{i_{x+1}}-v_{i_{x}}}\right)-\Phi\left(\frac{r_{i_{x}}-r_{i_{x-1}}}{v_{i_{x}}-v_{i_{x-1}}}\right)
$$

for all $i_{x}=i_{1}, \ldots, i_{m-1}$ in $\mathcal{S}^{+}$,

$$
P_{i_{m}}\left(\mathcal{N}^{+}\right)=1-\Phi\left(\frac{r_{i_{m}}-r_{i_{m-1}}}{v_{i_{m}}-v_{i_{m-1}}}\right)
$$

and

$$
P_{j_{y}}\left(\mathcal{N}^{+}\right)=0 \text { for all } j_{y} \in \mathcal{S}^{-}
$$

Given the fact that the total market size for the retailer $D$ is uncertain and characterized by the random variable $\Psi$ with pdf and $\operatorname{cdf} f(\cdot)$ and $F(\cdot)$, respectively, the primary demand (denoted by the superscript " 0 ") for all products $i \in \mathcal{S}^{+}$is $D_{i}^{0}=D P_{i}\left(\mathcal{N}^{+}\right)$and $D_{j}^{0}=0$ for all $j \in \mathcal{S}^{-}$. A primary demand of zero does not automatically mean that this product will not be sold. It may be that (depending on the stocking levels $Q_{i}$ for all $i \in \mathcal{N}$ ) another product $i \in \mathcal{S}^{+}$will be sold out in the middle of the selling season and some customers substitute a product from $\mathcal{S}^{-}$instead, or, a product is not in the assortment at all, at that moment substitution also occurs. In the following, the substitution process is described in detail. 


\subsubsection{Substitution process}

We consider that all customers can have multiple substitution attempts, i.e., if a customer's first-choice is not available, she is trying to buy her second choice having a positive net-utility. If the second choice is not available either (i.e. not in the assortment or out-of-stock), the customer tries to buy the third-choice product if available, and so on. If all products with positive purchasing probabilities are unavailable, then the customer does not buy and the demand is lost.

The majority of papers studying OOA or OOS based substitution only consider a single substitution attempt, i.e., after all primary demand is fulfilled, the remaining imbalance of leftovers and stock outs can partially be fulfilled via a single substitution attempt (Smith \& Agrawal, 2000; Netessine \& Rudi, 2003; Schlapp \& Fleischmann, 2018). This single substitution attempt is generally characterized by an exogenous substitution fraction matrix $\Gamma=\left(\gamma_{j i}\right)^{N+1 \times N+1}$ where $\gamma_{j i}$ denotes fraction of unmet $j$ demand that is willing to substitute product $i$ (substitution fraction). Assuming there is only a single substitution attempt and that the substitution fractions are constant and exogenous, the effective demand for product $i$ would be the primary demand plus all single-attempt substitution, i.e., $D_{i}^{e}=D_{i}^{0}+\sum_{j \neq i} \gamma_{j i}\left(D_{j}^{0}-Q_{j}\right)^{+}$as characterized in many previously studied models, e.g. Netessine \& Rudi (2003) and Schlapp \& Fleischmann (2018). However, in reality this assumption is rather restrictive since customers have normally a positive net-utility from more than one or two products and would therefore perform multiple substitution attempts.

In this study, we allow customers to make multiple substitution attempts and additionally, we do not assume any exogenous substitution fractions but consider endogenous substitution fractions. More specifically, we consider that the substitution probabilities depend on the substitution round and the remaining products available at each substitution round. While a substitution attempt is defined from a customer's point of view who has a specific product ranking in mind (Honhon \& Seshadri, 2013), a substitution round is rather seen from a modelling perspective as such that it first satisfies all primary demand. The resulting imbalance of leftover inventory of some products and unmet demand of others leads to the first substitution round. After satisfying the demand of the first substitution round, there may be still an imbalance of products with leftover inventory and products with unmet demand, which leads to the second substitution round, and so on, until all demand is met or no consumer finds a product for which he has a positive utility. The substitution probabilities on each round are endogenous, i.e., they are determined by the choice probabilities, which depend on the remaining products available on each substitution round. The following example will illustrate the choice process and the substitution process. 
Example 1: Suppose a firm stocks three products with $Q_{1}, Q_{2}, Q_{3}>0$, i.e., $\mathcal{N}_{0}^{+}=$ $\{1,2,3\}$ and $\mathcal{N}_{0}^{-}=\{\emptyset\}$. All three products fulfil the necessary and sufficient condition for being in $\mathcal{S}^{+}$and thus having strictly positive purchasing probabilities. Figure 4.1 shows the ranges of $\theta$ in which product 1, 2, and 3 are purchased. In section 1, customers obtain the highest net utility from buying product 1 , in section 2 , customers will buy product 2 , and in section 3 they will buy product 3 . Customers with a $\theta \in\left[\underline{\theta}, r_{1} / v_{1}\right)$ have a negative purchasing probability from all three products and thus choose the 'outside' option e.g., they leave the shop without a product. Therefore, the primary demand for not buying, products 1,2 , and 3 are $D_{0}^{0}=\Phi\left(\frac{r_{1}}{v_{1}}\right), D_{1}^{0}=\Phi\left(\frac{r_{2}-r_{1}}{v_{2}-v_{1}}\right)-\Phi\left(\frac{r_{1}}{v_{1}}\right), D_{2}^{0}=$ $\Phi\left(\frac{r_{3}-r_{2}}{v_{3}-v_{2}}\right)-\Phi\left(\frac{r_{2}-r_{1}}{v_{2}-v_{1}}\right)$, and $D_{3}^{0}=1-\Phi\left(\frac{r_{3}-r_{2}}{v_{3}-v_{2}}\right)$, respectively. Suppose the demands and order quantities are such that after satisfying the primary demand only product 2 is OOS (with some unmet demand) while product 1 and 3 are still available (Figure 4.2a), i.e., $\left(D_{i}^{0}-Q_{i}\right)<0$ for $i=1,3$ and $\left(D_{2}^{0}-Q_{2}\right)>0$. The updated sets of available and non-available products before the first substitution round are $\mathcal{N}_{1}^{+}=\{1,3\}$ and $\mathcal{N}_{1}^{-}=$ $\{2\}$. Thus, some customers who initially preferred product 2 will now substitute towards product 1 (section $2 \mathrm{a}$ in Figure $4.2 \mathrm{a}$ ) and product 3 (section $2 \mathrm{~b}$ in Figure $4.2 \mathrm{a}$ ). The 'first-round' substitution probabilities can be derived from the purchasing probabilities in the following way:

$$
\gamma_{21}^{1}\left(\mathcal{N}_{1}^{+}\right)=\frac{\Phi\left(\frac{r_{3}-r_{1}}{v_{3}-v_{1}}\right)-\Phi\left(\frac{r_{2}-r_{1}}{v_{2}-v_{1}}\right)}{\Phi\left(\frac{r_{3}-r_{2}}{v_{3}-v_{2}}\right)-\Phi\left(\frac{r_{2}-r_{1}}{v_{2}-v_{1}}\right)} \text { and } \gamma_{23}^{1}\left(\mathcal{N}_{1}^{+}\right)=\frac{\Phi\left(\frac{r_{3}-r_{2}}{v_{3}-v_{2}}\right)-\Phi\left(\frac{r_{3}-r_{1}}{v_{3}-v_{1}}\right)}{\Phi\left(\frac{r_{3}-r_{2}}{v_{3}-v_{2}}\right)-\Phi\left(\frac{r_{3}-r_{1}}{v_{3}-v_{1}}\right)}
$$

For vertically differentiated products, substitution only occurs to the 'adjacent available' products such that no customers substitute the outside option in this case, i.e., $\gamma_{20}^{1}\left(\mathcal{N}_{1}^{+}\right)=$ 0 . Suppose after the first substitution round, product 1 becomes OOS while product 3 is still available, i.e., $\mathcal{N}_{2}^{+}=\{3\}$ and $\mathcal{N}_{2}^{-}=\{2,1\}$ (see Figure 4.2b). Thus, some customers that preferred product 2 in the first substitution round, now substitute to product 3 (section $1 \mathrm{~b}$ in Figure 4.2b) or leave the shop without a product (section 1a in Figure $4.2 \mathrm{~b})$. Therefore, the 'second-round' substitution probabilities are

$$
\gamma_{10}^{2}\left(\mathcal{N}_{2}^{+}\right)=\frac{\Phi\left(\frac{r_{3}}{v_{3}}\right)-\Phi\left(\frac{r_{1}}{v_{1}}\right)}{\Phi\left(\frac{r_{3}-r_{1}}{v_{3}-v_{1}}\right)-\Phi\left(\frac{r_{1}}{v_{1}}\right)} \text { and } \gamma_{13}^{2}\left(\mathcal{N}_{2}^{+}\right)=\frac{\Phi\left(\frac{r_{3}-r_{1}}{v_{3}-v_{1}}\right)-\Phi\left(\frac{r_{3}}{v_{3}}\right)}{\Phi\left(\frac{r_{3}-r_{1}}{v_{3}-v_{1}}\right)-\Phi\left(\frac{r_{1}}{v_{1}}\right)} .
$$

One can see that the substitution probabilities are not constant but depend on the remaining products available, which changes every substitution round. Let $k=1, \ldots, K$ denote the number of substitution rounds where $K$ is a sufficient large number which guarantees that after $K$ rounds either all demand is satisfied or all remaining demand has a nonpositive net utility from the remaining products available. Furthermore, let $\gamma_{j_{m} i_{n}}^{k}\left(\mathcal{N}_{k}^{+}\right)$ 


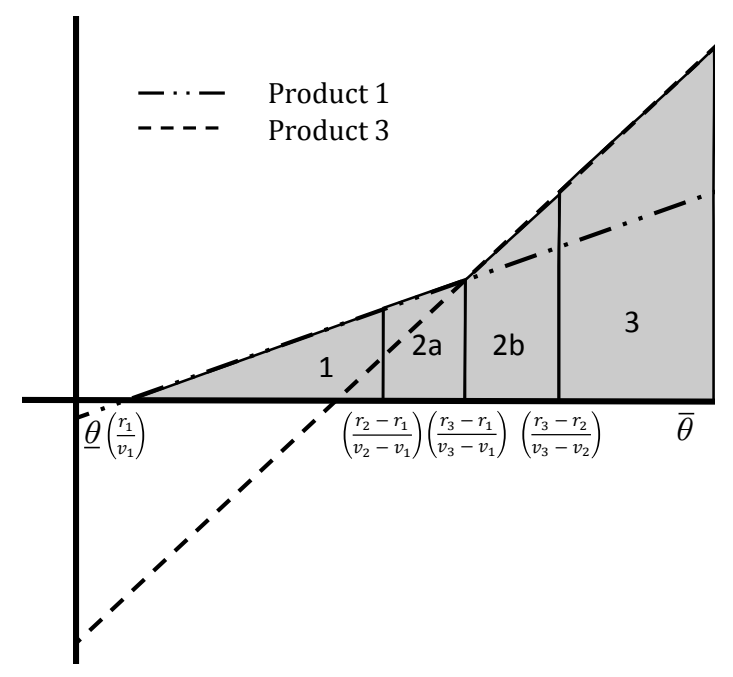

(a) Product 2 is out-of-stock

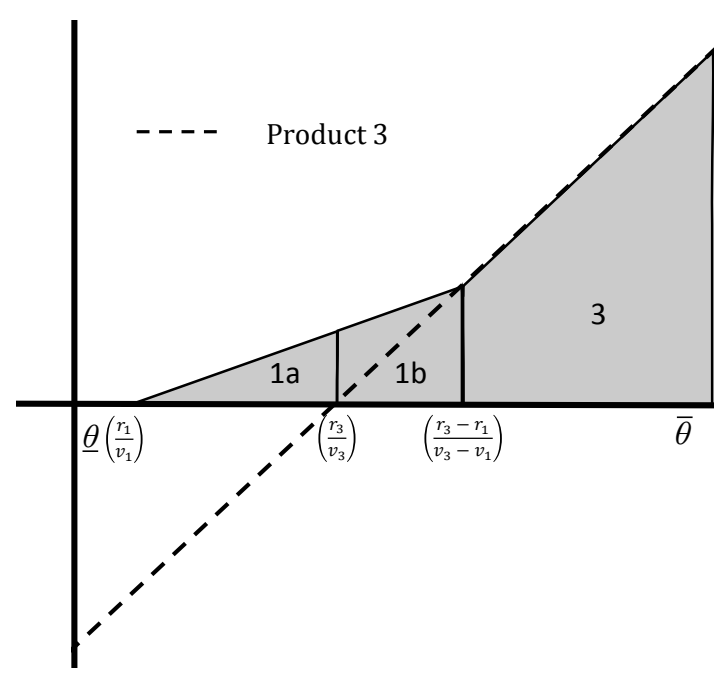

(b) Product 1 and 2 are out-of-stock

Figure 4.2: Fractions of consumers that would purchase a product, given the availability of the products

denote the substitution probability from product $j_{m} \in \mathcal{N}_{k}^{-}$to product $i_{n} \in \mathcal{N}_{k}^{+}$at the $k t h$ substitution round given a set of available products $\mathcal{N}_{k}^{+}$. This substitution probability can be formalized as follows

$$
\gamma_{j_{m} i_{n}}^{k}\left(\mathcal{N}_{k}^{+}\right)= \begin{cases}\frac{\Phi\left(\frac{r_{i_{n+1}}-r_{i_{n}}}{v_{i_{n+1}}-v_{i_{n}}}\right)-\Phi\left(\frac{r_{j_{m}}-r_{i_{n}}}{v_{j_{m}}-v_{n}}\right)}{\Phi\left(\frac{r_{i_{n}+1}-r_{j_{m}}}{v_{i_{n+1}}-v_{j_{m}}}\right)-\Phi\left(\frac{r_{j_{m}}-r_{i_{n}}}{v_{j_{m}}-v_{i_{n}}}\right)} & \text { if } i_{n} \text { is an available neighbour for } j_{m} \\ 0 & \text { otherwise. }\end{cases}
$$

Consequently, the effective demand considering multiple substitution rounds (and substitution attempts) is

$$
D_{i}^{e}=D_{i}^{0}+\sum_{k=1}^{K} \sum_{j \neq i} \gamma_{j i}^{k}\left(\mathcal{N}_{k}^{+}\right)\left(D_{j}^{k-1}-Q_{j}^{k-1}\right)^{+}
$$

where $D_{j}^{k-1}$ is the remaining demand of product $j$ after $k-1$ substitution rounds and $Q_{j}^{k-1}$ is the remaining inventory level of product $j$ after $k-1$ substitution rounds (note: $Q_{j}^{0}=Q_{j}$ ). Hence, the retailer's optimization problem becomes

$$
\max _{Q_{1}, \ldots, Q_{N}} \Pi=\sum_{i \in \mathcal{N}}\left(r_{i} \mathbb{E}\left[\min \left\{Q_{i}, D_{i}^{0}+\sum_{k=1}^{K} \sum_{j \neq i} \gamma_{j i}^{k}\left(\mathcal{N}_{k}^{+}\right)\left(D_{j}^{k-1}-Q_{j}^{k-1}\right)^{+}\right\}\right]-c_{i} Q_{i}\right) .
$$




\subsection{Solution procedure}

It has been shown that multi-product inventory problems with customer-driven stockout based substitution are inherently difficult to solve. So far, there exist no efficient algorithms finding the optimal solution to this problem. Moreover, the computational complexity is exponential in the number of products, hence heuristic procedures are developed to find (near) optimal stock level for $N$ products.

\subsubsection{Heuristic}

The heuristic procedure we propose iteratively solves a two-product case. The problem is solved in $N-1$ iterations, where in every iteration $g$, the problem is reduced to a two-product problem. The two products optimized in a single iteration are (i) the product $g$ and (ii) an aggregated group of products $R$, consisting out of all higher valued products. With the exception of the first iteration, more than two products are included, as the lower valued products are taken into account, but only two products are optimized. For every iteration $g$, the inventory level for product $g$, and the aggregated group $R$, is optimized with equation (4.10), under the stock levels already found in earlier iterations. Aggregation of the cost parameters, for group $R$ is based on the weighted average of the aggregated group, where the weighting factor is determined by probability the consumer chooses product $i, P_{i}$. By defining the cost parameters of the aggregated group as such, the actual costs or revenue made for the $N$-product problem is properly approximated. The substitution fractions $\gamma_{i j}^{k}\left(\mathcal{N}_{k}^{+}\right)$are defined by the substitution fractions between product $g$ and the 'adjacent' product which is part of the aggregated group. Moreover, the downward substitution equals the downward substitution in the $N$-product problem. Using these substitution fractions, the structure of the two-product problem is as close as possible to the original problem, which would not be the case when (weighted) averages of the parameters are used to calculate the substitution fractions between product $g$ and the aggregated group $R$. The heuristic starts with the lowest valued product, i.e. product 1. By starting with this product, the substitution fractions towards the no-purchase option are included properly. The following example will demonstrate the aggregation and sequence of the heuristic.

Example 2: Suppose there are four products and thus we need to determine $Q_{1}, \ldots, Q_{4}$, which is done in three iterations.

1. In the first iteration $(g=1)$, the heuristic starts with optimising the order quantity $Q_{1}$ and the quantity for an aggregated group $Q_{R}$ which consists of product 2,3 and 
4. The probability a consumer chooses product 1 or the aggregated product group $R$, are determined by the purchasing probabilities of the $N$-product problem, thus $P_{1}=P_{1}$ and $P_{R}=\sum_{i=2}^{4} P_{i}$. The cost parameters, $c_{i}$ and $r_{i}$ are determined by a weighted average, with the weighting factor $P_{i}$, which leads to $c_{R}=\frac{\sum_{i=2}^{4} P_{i} c_{i}}{\sum_{i=2}^{4} c_{i}}$ and $r_{R}=\frac{\sum_{i=2}^{4} P_{i} r_{i}}{\sum_{i=2}^{4} r_{i}}$. The substitution fraction, $\gamma_{1 R}^{k}\left(\mathcal{N}_{k}^{+}\right)$, between product 1 and the aggregated group $R$ is determined by the substitution fraction between product 1 and product 2 following from equation (4.8): $\gamma_{12}^{k}\left(\mathcal{N}_{k}^{+}\right)$. Downward substitution, from the aggregate product $R$ to product 1 is given by $\gamma_{R 1}^{k}\left(\mathcal{N}_{k}^{+}\right)=1$. After all parameters are set, a complete enumeration for a limited search space is performed to find the optimal $Q_{1}$ and $Q_{R}$ for these settings. Because a problem with $N=2$ might not even quasi-concave (Netessine \& Rudi, 2003), a closed form expression cannot be used to find the optimal solution and a search is needed to find the optimal solution. For every iteration the search space is limited by an upper bound for both products $g$ and $R$, given by the newsvendor model. The upper bound of $g, \bar{Q}_{g}$, is given by

$$
\bar{Q}_{g}=D P_{g}+D P_{R}
$$

The upper bound for the inventory level of the aggregated group $R$ is given by

$$
\bar{Q}_{R}=D P_{R}+\gamma_{g R}^{k}\left(\mathcal{N}_{k}^{+}\right) D P_{g}
$$

With these upper bounds, the search is performed. After finding the values of $Q_{1}$ and $Q_{R}$, the value of $Q_{1}$ is fixed and the next iteration starts $(g=2)$.

2. In the second iteration the value of $Q_{2}$ and the value $Q_{R}$ for the aggregated group, consisting of product 3 and 4 are optimized. The value of $Q_{1}$, as found in the previous iteration, remains unchanged. The substitution fraction $\gamma_{2 R}^{k}\left(\mathcal{N}_{k}^{+}\right)$is given by $\gamma_{23}^{k}\left(\mathcal{N}_{k}^{+}\right)$, and $\gamma_{R 2}^{k}\left(\mathcal{N}_{k}^{+}\right)=1$ for the downward substitution. The substitution between product 1 and product 2 is defined by equation (4.8). The purchasing probability $P_{R}$ is now given by $\sum_{i=3}^{4} P_{i}$. The cost parameters of the aggregated group $R$ are given by: $c_{R}=\frac{\sum_{i=3}^{4} P_{i} c_{i}}{\sum_{i=3}^{4} c_{i}}$ and $r_{R}=\frac{\sum_{i=3}^{4} P_{i} r_{i}}{\sum_{i=3}^{4} r_{i}}$. Again, a complete enumeration with a limited search space is performed to find the optimal values of $Q_{2}$ and $Q_{R}$ with the given value of $Q_{1}$, with the upper bounds of the search space defined by equation (4.11) and (4.12). After fixing the value of $Q_{2}$, the third, and last, iteration starts.

3. As for the last iteration only two products are left, aggregation is not needed any more. Under the fixed stock quantities of product 1 and 2 , the $Q_{i}$-levels for product 3 and 4 are determined by complete enumeration, with the upper bounds defined by equation (4.11). 
In most cases, the heuristic results in solutions very close to the optimal solution. Due to the combination of the discrete values of the inventory levels of $Q_{i}$ and the small values of total demand $D$, the heuristic can be one item off from the optimal solution. A neighbourhood search is therefore applied to obtain the right values.

By using this heuristic procedure, the problem complexity reduces to $O\left(n^{2}\right)$, which is significantly less compared to the complete enumeration of the $N$-product case $\left(O\left(2^{n}\right)\right)$.

\subsubsection{Solution method for cases with low demand uncertainty}

The heuristic procedure described in the previous section does work well, e.g. it almost always finds the (near) optimal solution. However, when the coefficient of variation is low $(C V=0.33)$, different stock quantities are found for the lowest valued product, when the optimal solution is to not have this product in stock. For vertically differentiated products, there is always complete downward substitution, if higher valued products are not available. In the first iteration of the heuristic, when there are only 2 products considered, it is therefore unlikely to not have the lowest valued product in stock, although this is optimal for the $N$-product problem. With vertically differentiated products, there is always full substitution downwards, when the higher valued product is not available. Hence, the lowest valued product will be bought when there are no other products available, which makes it beneficial to have this product in stock when demand is uncertain. However, when the demand uncertainty is low, the need for a low-cost back-up product is decreased, as the demand estimation can be done more accurately. In the two-product case, there is not another back-up product and thus in this iteration the heuristic proposes to have the lowest product in stock, even though this is not optimal in the $N$-product problem.

For these instances, an extra step is required. Besides using the heuristic as described above, another run is needed, where the value of $Q_{1}$ is fixed to zero at the beginning. This could be done for the first, second, third, $N-2$ product. To find the stock quantities for the products, which are not fixed to zero, the same procedure as heuristic is used. By comparing the expected profit levels for all these runs, the optimal solution is found. The complexity of the problem is increased to $O\left(n^{3}\right)$ for this extension.

\subsection{Numerical results}

All numerical examples presented in this section, consider a set of four products $(N=$ 4) with the consumer quality valuation $(\Theta)$ of the products between $[0,1)$. The total demand (sum of demand of all four products) follows a negative binomial distribution with mean $\mu$ and standard deviation $C V * \mu$. Three values of $\mu$ are chosen: 10, 15, and 
Table 4.1: Profit and stock levels $(Q)$ for the optimal solution of the basic example, found with complete enumeration $(\mathrm{CE})$ and the optimality gap and stock levels $(Q)$ for the heuristic and the extension of the heuristic.

\begin{tabular}{ll|rcrccc}
\hline & & \multicolumn{2}{|c}{ CE } & \multicolumn{2}{c}{ Heuristic } & \multicolumn{2}{c}{ Extended version } \\
$\mu$ & $\mathrm{CV}$ & Profit & $\left(Q_{1}, . ., Q_{4}\right)$ & $\mathrm{GAP}$ & $\left(Q_{1}, . ., Q_{4}\right)$ & $\mathrm{GAP}$ & $\left(Q_{1}, . ., Q_{4}\right)$ \\
\hline 10 & 0.33 & $€ 18.72$ & $(0,5,3,1)$ & $1.5 \%$ & $(5,1,3,1)$ & $0 \%$ & $(0,5,3,1)$ \\
10 & 0.50 & $€ 15.34$ & $(5,1,3,1)$ & $0 \%$ & $(5,1,3,1)$ & $0 \%$ & $(5,1,3,1)$ \\
10 & 0.67 & $€ 12.42$ & $(6,1,2,1)$ & $0 \%$ & $(6,1,2,1)$ & $0 \%$ & $(6,1,2,1)$ \\
\hline 15 & 0.33 & $€ 28.47$ & $(0,7,4,2)$ & $0.3 \%$ & $(6,2,5,2)$ & $0 \%$ & $(0,7,4,2)$ \\
15 & 0.50 & $€ 23.26$ & $(6,2,4,2)$ & $0 \%$ & $(6,2,4,2)$ & $0 \%$ & $(6,2,4,2)$ \\
15 & 0.67 & $€ 18.80$ & $(7,2,4,1)$ & $0 \%$ & $(7,2,4,1)$ & $0 \%$ & $(7,2,4,1)$ \\
\hline 20 & 0.33 & $€ 38.02$ & $(7,3,6,3)$ & $0 \%$ & $(7,3,6,3)$ & $0 \%$ & $(7,3,6,3)$ \\
20 & 0.50 & $€ 31.27$ & $(8,3,6,2)$ & $0 \%$ & $(8,3,6,2)$ & $0 \%$ & $(8,3,6,2)$ \\
20 & 0.67 & $€ 25.13$ & $(11,2,4,2)$ & $0 \%$ & $(11,2,4,2)$ & $0 \%$ & $(11,2,4,2)$ \\
\hline
\end{tabular}

20. For each value of $\mu$ we consider three levels of demand uncertainty as reflected by the coefficient of variation: $C V=0.33,0.5$, and 0.67 . The experiments are carried out with Matlab2018a.

The following basic example is used for the numerical analysis. Let, $v_{i}=\{35,43,50,55\}$, $r_{i}=\{3,5,8,12\}$ and $c_{i}=\{1.2,2.5,4.8,8.4\}$, such that every product has a positive purchasing probability and thus could be present in the optimal assortment.

\subsubsection{Performance of heuristic}

The results of the basic example are given in Table 4.1. For both the heuristic and the extended version, the optimality gap, the found stock levels $\left(Q_{i}\right)$ are given. In seven experiments, the first heuristic already gives the optimal solution; however, for two, the more extended solution method is required. In these cases the optimal stock level of the first is zero (i.e. $Q_{1}=0$ ). As explained before, for vertically differentiated products there is always full downward substitution. When demand uncertainty is high, the lowest valued product serves as the back-up product, i.e. this product will be sold when the others are OOS. Moreover, the lowest valued product is the cheapest to have in stock for the retailer and therefore profit losses are minimized in the case of overstocking this product. When demand uncertainty decreases, the risk of overstocking decreases and it is acceptable to use a higher valued and more expensive product as back up.

Besides the expected trends, such as an increase in items in stock with a higher average 
demand and a decreased expected profit with higher demand variation, some interesting results are found. For a demand $\mu=10$ with a low CV $(0.33)$, it is optimal to not have product 1 in stock. Where, if $\mu$ increases, product 1 has the highest inventory level. For vertically differentiated product, there is always full downward substitution. Therefore, a buffer occurs for the lowest valued product in stock. When it is optimal to have all products in stock, the buffer occurs at product 1 . However, when product one is not in the optimal assortment, the buffer occurs at product 2. This has an effect on the breadth of the assortment, as only three out of four products are offered. However, the total number of items offered hardly differs, when three products are in the optimal assortment, the total inventory is reduced with only one item.

Structural differences are found in comparison with the newsvendor model without substitution. For the basic example, the probability consumers purchase product 3 is the highest. The newsvendor model therefore, gives the highest inventory levels for product 3. Second highest inventory is present for product 1, which has the highest critical ratio. However, when substitution is included, the most items are in stock of product 1 . Due to the substitution between products, the risk of overstocking decreases, as demand is pooled between the different products. With the vertically differentiated products, there is always substitution towards a lower valued product when higher valued products are not available (any more). Thus, it becomes profitable to have more of the lowest valued product in stock, as the chances of selling all of them increase.

\subsubsection{Assortment decisions}

We compare our algorithm with the algorithm of Pan \& Honhon (2012), which optimizes the assortment based on the profit margins. As this algorithm does not include inventory levels, we optimize the inventory levels of the products in the assortment, according to the algorithm of Pan \& Honhon (2012), with our own approach. The results are presented in Table 4.2. The major difference is found in the assortment. When inventory levels are not included, the optimal assortment for the basic example only includes product 4 . For this product, there is a positive purchasing probability of $78 \%$, hence the total inventory levels and the expected profit will be affected. As the number of products decrease, less products will be sold and profit levels decrease heavily, in some cases with more than 20\%. Moreover, more consumers will leave the shop without a product, which will probably have a negative effect in the long term on the consumer loyalty. This comparison clearly show the importance of including inventory levels in decisions regarding the optimal assortment. 
Table 4.2: Comparison of the results given by the algorithm of Pan \& Honhon (2012) and our approach. The table presents the optimal stock levels $\left(Q_{1}, . ., Q_{4}\right)$, the differences between total stock levels $\left(\triangle \sum Q_{i}\right)$ and the reduction in profit $(\triangle$ Profit $)$.

\begin{tabular}{cc|cccc}
\hline & & With inventory consideration & \multicolumn{3}{l}{ Without inventory consideration } \\
$\mu$ & $\mathrm{CV}$ & $\left(Q_{1}, . ., Q_{4}\right)$ & $\left(Q_{1}, . ., Q_{4}\right)$ & $\triangle \sum Q_{i}$ & $\triangle$ Profit \\
\hline 10 & 0.33 & $(0,5,3,1)$ & $(0,0,0,6)$ & -1 & $-6.25 \%$ \\
10 & 0.50 & $(5,1,3,1)$ & $(0,0,0,5)$ & -5 & $-14.28 \%$ \\
10 & 0.67 & $(6,1,2,1)$ & $(0,0,0,4)$ & -6 & $-23.99 \%$ \\
\hline 15 & 0.33 & $(0,7,4,2)$ & $(0,0,0,10)$ & -1 & $-6.74 \%$ \\
15 & 0.50 & $(6,2,4,2)$ & $(0,0,0,8)$ & -6 & $-13.21 \%$ \\
15 & 0.67 & $(7,2,4,1)$ & $(0,0,0,7)$ & -6 & $-22.16 \%$ \\
\hline 20 & 0.33 & $(7,3,6,3)$ & $(0,0,0,13)$ & -6 & $-6.20 \%$ \\
20 & 0.50 & $(8,3,6,2)$ & $(0,0,0,11)$ & -8 & $-13.32 \%$ \\
20 & 0.67 & $(11,2,4,2)$ & $(0,0,0,9)$ & -10 & $-21.78 \%$ \\
\hline
\end{tabular}

\subsubsection{Single vs. multiple substitution round}

As mentioned in Section 4.2, commonly only one round of substitution is included in research on assortment planning or inventory decisions under consumer driven substitution. Although several studies mention that it is not too restrictive to use a single substitution round instead of multiple rounds (Smith \& Agrawal, 2000; Hübner et al., 2016), Table 4.3 shows the opposite. Indeed, Kök \& Fisher (2007) show that multiple substitution rounds can be approximated by a single one with an increased substitution fraction. However, in our approach with the vertically differentiated products, an increased substitution fraction for a single round will be arbitrary compared to equation (4.8).

The results of the comparison shown in Table 4.3 is obtained step-wise. The inventory levels $Q_{i}$ are calculated with a single substitution round, instead of the multiple rounds. However, in real life, multiple substitution rounds will take place, also when during the optimization only a single substitution round is taken into account. To obtain the profit differences, the inventory levels found for the single substitution round are therefore implemented in the model that considers multiple substitution rounds to find the profit levels. These profit levels are then compared with the profit values which belong to the optimal $Q_{i}$ levels for the multiple substitution rounds.

As the results show, profit reduction can be significant, sometimes more than $6 \%$. The main differences occur due a reduction in total inventory level $\left(\triangle \sum Q_{i}\right)$. When multiple substitution rounds are included, total $Q_{i}$ levels are in most cases higher, and more products are included in the optimal assortment. For one experiment $(\mu=15, C V=0.33)$ 
Table 4.3: Comparison of the optimal stock levels $\left(Q_{1}, . ., Q_{4}\right)$ for a single substitution round and multiple substitution rounds and the differences between total stock levels $\left(\triangle \sum Q_{i}\right)$ and the reduction in profit ( $\triangle$ Profit).

\begin{tabular}{cc|cccc}
\hline & & Multiple & \multicolumn{3}{|c}{ Single } \\
$\mu$ & $\mathrm{CV}$ & $\left(Q_{1}, . ., Q_{4}\right)$ & $\left(Q_{1}, . ., Q_{4}\right)$ & $\triangle \sum Q_{i}$ & $\triangle$ Profit \\
\hline 10 & 0.33 & $(0,5,3,1)$ & $(0,3,4,1)$ & -1 & $-0.48 \%$ \\
10 & 0.50 & $(5,1,3,1)$ & $(0,3,4,1)$ & -2 & $-3.09 \%$ \\
10 & 0.67 & $(6,1,2,1)$ & $(0,3,3,1)$ & -1 & $-6.47 \%$ \\
\hline 15 & 0.33 & $(0,7,4,2)$ & $(0,5,6,2)$ & 0 & $-1.72 \%$ \\
15 & 0.50 & $(6,2,4,2)$ & $(0,4,6,1)$ & -3 & $-3.44 \%$ \\
15 & 0.67 & $(7,2,4,1)$ & $(3,2,5,1)$ & -3 & $-5.71 \%$ \\
\hline 20 & 0.33 & $(7,3,6,3)$ & $(0,6,8,2)$ & -3 & $-1.47 \%$ \\
20 & 0.50 & $(8,3,6,2)$ & $(0,6,7,2)$ & -4 & $-3.27 \%$ \\
20 & 0.67 & $(11,2,4,2)$ & $(4,3,7,1)$ & -4 & $-5.19 \%$ \\
\hline
\end{tabular}

total stock level remains the same, however the stock quantities of the individual products change. Although more products are in stock of the more profitable product 3 , the expected profit is reduced for the single substitution round. This could occur due to the rounding to integer quantities for the stock levels. Total demand increase when multiple substitution rounds are included. When consumers only take one attempt/round to purchase a substitute, it could happen that the substitute product is also OOS. Thus, these consumers leave the shop without a product. When multiple substitution rounds are present, there is a higher chance the consumer will find a substitute product and less consumers will leave the shop without a product.

\subsubsection{Sensitivity analysis}

A sensitivity analysis is carried out to answer four questions:

1. What is the impact of the product differentiation in terms of quality attributes $v_{i}$

2. What is the impact of the product differentiation in terms of profit margin $\left(r_{i}-c_{i}\right)$

3. How does the critical ratio, of the newsvendor model, affect the assortment and inventory decisions

4. Can it be optimal to have products in stock with an initial purchasing probability of zero 
Table 4.4: Average profit and stock levels $\left(Q_{i}\right)$ for the average demand with decreased $(-10 \%)$ and increased $(+10 \%)$ quality $v_{i}$.

\begin{tabular}{c|cccc}
\hline & \multicolumn{2}{|c}{ Decreased quality attribute } & \multicolumn{2}{c}{ Increased quality attribute } \\
$\mu$ & Profit & $\left(Q_{1}, Q_{2}, Q_{3}, Q_{4}\right)$ & Profit & $\left(Q_{1}, Q_{2}, Q_{3}, Q_{4}\right)$ \\
\hline 10 & $€ 14.90$ & $(3.33,2.33,3,0.67)$ & $€ 15.92$ & $(4,2,2.33,1.33)$ \\
15 & $€ 22.83$ & $(5,3.67,4.33,0.67)$ & $€ 24.06$ & $(7,1.67,3.67,2.33)$ \\
20 & $€ 30.60$ & $(6.67,4.67,6,1)$ & $€ 32.21$ & $(6.67,4,4.67,3)$ \\
\hline
\end{tabular}

For both the quality attributes $v_{i}$ and the profit margin an in-/decrease of $10 \%$ is used of the values of the basic example. For the variation in profit margin, only $c_{i}$ is changed, to exclude the effect of a changing purchasing probability. To analyse the effect of the critical ratio, two cases are analysed. One where the critical ratio is equal for all products, and one where the critical ratio of product 1 is much lower than the basic example, and slightly altered for the other products.

\section{Effect of the quality attribute}

When products are closer to each other, or more differentiated in terms of the quality attribute $v_{i}$, the utility function is affected and thus the purchasing probability for the products changes. When the product quality increases, more consumers are willing to purchase a product, as more consumers have a positive utility for one of the products. Although this is indicated by the increase in profit, the total items in stock are not affected by a change in quality (see Table 4.4). On the other hand, when the products differentiate less, and thus the $v_{i}$ is decreased, a lower expected profit is obtained. The change in expected profit is obtained by a different distribution of the total items in stock over the four products. When products differentiate more from each other in terms of quality attribute $\left(v_{i}\right)$, the fraction of consumers that want to purchase product 4 increases, thus more of the most profitable product can be sold.

\section{Effect of the profit margin}

The analysis on product differentiation on the basis of profit margin only affects the retailers performance, but not the purchasing probabilities of the consumer as only the $\operatorname{costs} c_{i}$ is changed. A change in profit margin, directly changes the expected profit, as shown in Table 4.5. Although the profit margins are changed by $10 \%$, the actual change in expected profit is higher. A $16 \%$ increase is present at the moment the profit margins are increased, and a $15 \%$ decrease for the decreased profit margins, obtained by the change in 
Table 4.5: Average profit and stock levels $\left(Q_{i}\right)$ for the average demand with decreased $(-10 \%)$ and increased $(+10 \%)$ profit margin $r_{i}-c_{i}$.

\begin{tabular}{c|cccc}
\hline & \multicolumn{2}{|c}{ Decreased profit margin } & \multicolumn{2}{c}{ Increased profit margin } \\
$\mu$ & Profit & $\left(Q_{1}, Q_{2}, Q_{3}, Q_{4}\right)$ & Profit & $\left(Q_{1}, Q_{2}, Q_{3}, Q_{4}\right)$ \\
\hline 10 & $€ 13.22$ & $(3.33,2,2.33,1)$ & $€ 17.95$ & $(4.33,2.33,2.67,1)$ \\
15 & $€ 20.03$ & $(4.67,3,3.67,1.33)$ & $€ 27.23$ & $(7.67,2,4.33,1.67)$ \\
20 & $€ 26.86$ & $(8.33,2.33,5,2)$ & $€ 36.43$ & $(10,2.67,5.67,2.33)$ \\
\hline
\end{tabular}

Table 4.6: Average profit and stock levels $\left(Q_{i}\right)$ for the average demand with an equal critical ratio and various critical ratio.

\begin{tabular}{c|cccc}
\hline & \multicolumn{2}{|c}{ Equal critical ratio } & \multicolumn{2}{c}{ Various critical ratio } \\
$\mu$ & Profit & $\left(Q_{1}, Q_{2}, Q_{3}, Q_{4}\right)$ & Profit & $\left(Q_{1}, Q_{2}, Q_{3}, Q_{4}\right)$ \\
\hline 10 & $€ 41.32$ & $(0,0,0,9)$ & $€ 13.95$ & $(0,6.33,1.33,0.67)$ \\
15 & $€ 62.15$ & $(0,0,0,13)$ & $€ 21.05$ & $(0,9,2,1.33)$ \\
20 & $€ 82.90$ & $(0,0,0,17)$ & $€ 28.15$ & $(0,11.67,2.67,1.67)$ \\
\hline
\end{tabular}

the stock levels. When the profit margin decreases, the critical ratio also decreases, and it becomes less favourable to have items of these products in stock. When profit margins increase, the opposite effect is present.

\section{Effect of the critical ratio}

To analyse the effect of the critical ratio $(C R)$, two cases are evaluated. In the basic example, the critical ratio of the products decreased, from product 1 to $4\left(C R_{1}=0.6, C R_{2}=\right.$ $\left.0.5, C R_{3}=0.4, C R_{4}=0.3\right)$. For the first analysis, the critical ratio is set equal for each product, by adapting the cost parameter $\left(c_{i}\right)$. With $c_{i}=\{1.1,1.85,2.95,4.4\}$, and $r_{i}, v_{i}$ equal to the basic example $\left(v_{i}=\{35,43,50,55\}, r_{i}=\{3,5,8,12\}\right)$, the critical ratio of each product is 0.63 . Table 4.6 shows that at the moment all products have an equal critical ratio, the optimal inventory decision is to have product 4 in stock, as this has the highest profit margin. The high corresponding profit level are the result of the increased profit margin for the higher valued products.

In the second analysis, the critical ratio of product 1 is decreased, such that it is lower than the ratio for product 2 . The cost vector is changed to: $c_{i}=\{2.00,2.50,5.30,9.00\}$, $r_{i}$ and $v_{i}$ remain unchanged. The critical ratio for each product is now $0.33,0.5,0.34$ and 0.25 for product 1,2,3 and 4 respectively. Although all products still have an initial demand larger than 0 , product 1 is not in the optimal assortment any more, see Table 4.6. 
Due to the low profit margin, it becomes more beneficial to let consumers buy a substitute product or leave the shop without a purchase (those who substitute downward).

Although the profit margin does have a large effect on the optimal assortment and inventory decisions, the critical ratio also has a significant influence.

\section{Initial purchasing probability of zero}

The question rises whether a product without an initial demand $\left(D_{i}^{0}=0\right)$ can be present in the optimal assortment. The parameters of the basic example are changed, such that product 4 does not have a positive purchasing probability any more; however, it has an increased profit margin. Let $v_{i}$ be $\{35,43,50,53\}, r_{i}=\{3,5,8,12\}$ and $c_{i}=\{1.2,2.5,4.8,7.9\}$. Table 4.7 , shows the optimal stock quantities with these parameter settings. Even though there is no initial demand for product 4, with these profit margins it is optimal to have only this product in the assortment. Thus the decision to have certain products in stock not only depends on the initial demand, it also depends on the expected revenue and the fraction of consumers that substitute.

Table 4.7: Average profit and stock levels $\left(Q_{i}\right)$, with an initial purchasing probability of zero for product 4 .

\begin{tabular}{cc|cc}
\hline$\mu$ & $\mathrm{CV}$ & Profit & $\left(Q_{1}, . ., Q_{4}\right)$ \\
\hline 10 & 0.33 & $€ 20.80$ & $(0,0,0,7)$ \\
10 & 0.50 & $€ 16.05$ & $(0,0,0,6)$ \\
10 & 0.67 & $€ 11.91$ & $(0,0,0,5)$ \\
\hline 15 & 0.33 & $€ 31.47$ & $(0,0,0,10)$ \\
15 & 0.50 & $€ 24.30$ & $(0,0,0,9)$ \\
15 & 0.67 & $€ 18.10$ & $(0,0,0,7)$ \\
\hline 20 & 0.33 & $€ 42.05$ & $(0,0,0,13)$ \\
20 & 0.50 & $€ 32.54$ & $(0,0,0,12)$ \\
20 & 0.67 & $€ 24.36$ & $(0,0,0,10)$ \\
\hline
\end{tabular}

\subsection{Conclusion and discussion}

In this chapter, assortment and inventory decisions are simultaneously optimized for vertically differentiated products under consumer driven substitution. Substitution occurs when a product is not available, due to either a stock-out or because it is not included 
in the assortment. This study is one of the few studies in which assortment and inventory decisions are combined and multiple substitution rounds are allowed. Based on the demand structure for vertically differentiated products, a procedure is developed to determine the substitution fractions for every round of substitution. Moreover, in this study a heuristic is developed to find (near) optimal solutions for multiple products that belong to a product category.

Our numerical examples show the importance of considering substitution behaviour of consumers at the retailer. When combined inventory decisions are made for the products within a product category, rather than for the individual products separately, the optimal stock levels are increased. An increased stock level will lead to less stock-outs, thus to a higher service level and more satisfied consumers. Moreover, we show the importance of including multiple substitution rounds. Besides, we show how demand uncertainty affects the optimal decisions. Furthermore, the effect of the profit margin, the quality attribute of the product and the effect of the critical ratio (from the newsvendor problem) are investigated in this study.

In our study the OOA and OOS substitution fractions are considered equal, which is not necessarily true for a retailer, although the difference between them might be small. Facing stock-outs or excluding products from the assortment might cause long-term negative effects for a retailer, as consumers might change to another shop. For a retailer it will be important to keep this in mind; however, it is hard to measure this effect quantitatively.

The number of items that can be in stock only take integer values in this study. The demand values used for the numerical analysis are realistic for a grocery retailer, however they are relative small. Due to these relative small demand in the numerical examples, the assumption of integer stock quantities can influence the results. With an increase in demand, compared to the demand levels used for the numerical analysis, or by allowing a continuous stock level, this limitation can be eliminated. However, a complete enumeration, used to test the performance of the heuristic, will not be possible within a reasonable time any more. Furthermore, the deviation obtained by the integer values of the stock levels is limited.

In this chapter, a heuristic procedure is developed to support decisions on which and how many products of a product category to stock under consumer driven substitution. The heuristic is tested for an instance of four products; however, it can be easily extended for a larger product set due to the structure, which is based on a two-product problem. By aggregating most of the products, the inventory levels of a single product and the aggregated group can be determined. Regardless how many products are present in the aggregated group, this heuristic can deal with these larger instances. 
The heuristic is developed for vertically differentiated products. The change towards horizontally differentiated products is possible with the proposed heuristic, although some adoptions will be needed, as the substitution will not necessarily be towards 'adjacent' products.

Our study does not include a fix cost when a product is included in the assortment, as for example considered by Pan \& Honhon (2012). When this cost would be included, the results might change, as it is costly to include a small amount of items of the products. Thus, when a product is included in the assortment, the number of items to stock most likely increases, and a limited assortment will be offered.

The focus of this research is a single period setting, however at a retailer products are often in stock for multiple periods. To obtain settings that are more realistic for a retailer, the model can be extended to a multi-period problem. This study can serve as a starting point for such an extension, as the developed heuristic should be able to cope with a multiperiod setting. Moreover, the inventory levels found in this research might represent the quantity of products that the retailer should have at the start of a period; however, more research is required. 



\section{Chapter 5}

\section{Donation management for menu planning at soup kitchens}

This chapter is published as:

Buisman, M.E., Haijema, R., Akkerman, R., \& Bloemhof, J.M., (2019). Donation management for menu planning at soup kitchens. European Journal of Operations Research, 272. doi: 10.1016/j.ejor.2018.06.005 


\section{Abstract}

The food industry is confronted with a pressure to reduce waste and to make agreements on donating surplus food to charitable organizations. Charitable organizations such as food banks and soup kitchens can use these donations in preparing food parcels or meals for their clients. For soup kitchens, donation management is strongly influencing menu planning, and conversely, menu planning considerations have a strong impact on donation management decisions. To make the best use of (mostly highly perishable) food donations, we develop an MILP model for integrated donation management and menu planning that proposes a menu plan and suggests which (part of the) donations to accept. The combination of menu planning and donation management is essential for soup kitchens, but has not been studied before.

The model is used to assess the impact of contracts on a strategic or tactical level, and captures operational decision making due to the integration of donation management and menu planning. To deal with meal variety considerations and to resemble planning practices, the developed model is solved in a rolling horizon. The results show that (i) the use of donations reduces overall costs for the soup kitchen; (ii) despite the short shelf life of donations, most donations can be used efficiently; and (iii) meal variety can be easily ensured and food donations increase this variety. In addition to the benefits for soup kitchens, the approach has implications for waste reduction in food supply chains, by structural/contractual donations of surplus food by retailers. 


\section{Donation management and menu planning}

\subsection{Introduction}

Charitable organisations such as food banks and soup kitchens are important contributors to food and meal provision to socially isolated and poor people. In such organisations, one of the main ways to keep costs low is to use surplus food from retailers or food companies. For instance, supermarkets can have difficulties in aligning supply and demand for perishable products, and often order many products to prevent out-of-stock situations, potentially resulting in surplus food and large waste streams (Monier et al., 2010).

Companies find different solutions to cope with this surplus food, such as donating to soup kitchens or food banks, or conversion to bio gas (Lee \& Tongarlak, 2017). Minimizing food waste has recently also been put on the political agenda, for instance demonstrated in the French Government's introduction of a law that forbids supermarkets to waste food, and obliges them to sign contracts with charitable organisations for food donations (Chrisafis, 2016). Another example is a Californian law that limits the amount of organic waste companies can produce yearly (CalRecycle, 2017).

This chapter deals with donation management and menu planning at soup kitchens, which are institutions that mostly rely on donations and provide complete meals for people that require assistance, such as homeless people. One of the best-known charitable organizations providing these services is the Salvation Army. Due to the characteristics of food donations, i.e. varying products with a generally short shelf life, menu planning at soup kitchens is challenging. Products must be used shortly after they are donated, and be integrated in menu plans that aim at a varied diet. For soup kitchens, this implies fast decision making on whether they want to accept a food donation or not, depending on the amount of product, its shelf life, as well as its usefulness in menu planning. The soup kitchen wants to avoid accepting donations it is not able to use, to prevent wasting the donated product, as well as potential costs related to the collection of the donation. From a supply chain perspective, accepting a donation that will eventually still be wasted would just shift the food waste to another party in the food supply chain. Furthermore, it prevents other parties from using this donation. Whether products are useful mainly depends on the meal variety a soup kitchen wants to offer. Soup kitchen clients are often highly dependent on the provided meals (Wicks et al., 2006) and offering varied meals is a way to improve the nutritional intake of the clients (Wilson et al., 2004). Furthermore, it is also much more attractive to eat varied meals.

Donations to charitable organisations are usually made on a voluntarily basis. These organisations often do not know when and how much food they will receive. Using contracts between the charitable organisation and the donating party, such as recently enforced by a French law, could improve the handling of surplus food. When donations are regulated 
via contracts, soup kitchens are more aware of the food that will come in, possibly making it easier to use donations efficiently. Furthermore, it helps the donating party (e.g. a retailer) to better make use of the surplus food. Besides, contracts give the opportunity to regulate other factors such as transport, quantities, and shelf life.

Assessing available research on donation management for soup kitchens or food banks shows that the above-mentioned issues have not been addressed in the literature. Most available studies either address the characteristics of the client base relying on charitable organisations for food, or they address the nutritional aspects of the provided meals (Eppich \& Fernandez, 2004; Wicks et al., 2006; Sprake et al., 2014). Research focussing on donation management and menu planning for food banks or soup kitchens is not available.

In this chapter, we aim to evaluate the donation management at a soup kitchen on a tactical level. In order to evaluate tactical decisions effectively, an integration of operational decisions is required. Therefore we develop a decision support approach integrating menu planning in decision-making for donation management, to be able to make the best use of food donations in soup kitchens while assuring meal variety for the clients. Applying a rolling horizon approach, we study the impact of contractual and managerial issues and characteristics of fresh food donations on the performance of a soup kitchen in terms of costs, product waste, meal variety, and the donation acceptance rate. The problem and results give rise to some decision rules that are tested. As input to a managerial discussion the effect of different types of contracts, donation characteristics (e.g. shelf life), the preferred meal variety, and several cost parameters are studied.

The remainder of this chapter is organized as follows. Section 5.2 discusses related literature on donation management and menu planning. Section 5.3 then provides our mathematical modelling approach. The experimental design is subsequently stated in Section 5.4 and our results are discussed in Section 5.5. In Section 5.6 the MILP results are compared with some decision rules for ad hoc donations. Finally, the chapter discusses conclusions and managerial insights in Section 5.7.

\subsection{Related literature}

In this section, we give an overview of related literature. In general, two streams of literature are related to our research: menu planning and donation management. 


\section{Donation management and menu planning}

\subsubsection{Menu planning}

Studies related to menu planning are widely available. The first study providing decision support in this area is the seminal work by Stigler (1945). When computers came into use, Balintfy (1964) was one of the first who solved menu planning by computer. He developed an integer programming model to determine the optimal menu planning by minimizing costs while considering dietary constraints. Over time, these type of menu planning models have become more advanced, e.g. by including meal production scheduling decisions (Guley \& Stinson, 1984). An extended review of menu planning can be found in the research of Lancaster (1992); here, we limit ourselves to briefly outlining some recent developments.

In general, there are three generations of menu planning models (Lancaster, 1992). The first generation of menu planning models focuses on cost minimization, the second generation on consumer preferences, and the third generation on individual consumers. In recent years, the focus of dietary problems is mainly on nutritional recommendations, either for humans or animals. For instance, Oishi et al. (2011) developed a linear programming model to optimize feed systems for cattle based on minimizing costs, as well as nitrogen and phosphorus intake. Also, Cadenas et al. (2004) implemented a modelling approach to solve a diet problem at Argentinian farms to minimize costs, while considering the nutritional recommendations. However, models for animal feed do not include palatability or meal variety constraints as is often the case in menu planning for humans. For instance, Leung et al. (1995) developed a mixed integer linear programming (MILP) model to optimize a diet for one week, fulfilling the nutritional recommendations and minimizing costs or cooking time. They reduced complexity by using recipes instead of separate food ingredients. Also, Seljak (2009) introduced the diet problem as a multi-criteria knapsack problem, which she solved with an evolutionary algorithm. More recently, Bas (2014) developed an MILP model to minimize the glycemic load of the daily optimal serving sizes. She used robust optimization to deal with the uncertainty in the measured glycemic index of food items.

Although menu planning can be applied in different settings such as catering services and hospitals, no research dealt with soup kitchens and donation management. Food donations obviously impact menu planning decisions, but including donations will result in a more complex decision problem because of the short shelf life of donated food products. They need to be used soon after donation, limiting the options for menu planning. 


\subsubsection{Donation management at soup kitchens or food banks}

Previous research on donation management for food banks and soup kitchens is mostly related to (i) planning and scheduling issues such as vehicle routing and allocation problems and (ii) nutritional aspects of food donations and meals provided at soup kitchens.

The planning and scheduling issues mostly focus on the design and operation of efficient transportation networks. Ghoniem et al. (2012) solved a vehicle routing problem where a central depot serves several customers while balancing transportation distances for the charitable organisation and the travel distances for customers. Davis et al. (2014) propose a system where all donated products flow through satellite locations (food delivery points), especially dealing with perishability and food safety. Balcik et al. (2014) describe a multivehicle sequential allocation problem for collecting and delivering food donations. Their research is an extension of the model developed by Lien et al. (2014), which only dealt with single routes, and could be applied to either food banks or soup kitchens. Solak et al. (2014) studied a location-routing problem with the determination of delivery sites at which agencies pick up food items. Analysing the donation patterns offered to a food bank is done by Brock \& Davis (2015). They used four different forecasting methods to predict the supply of donation to food banks, concluding that the forecasting methods tend to overestimate the future supply. Finally, Orgut et al. (2015) developed mathematical models to distribute food from a central location of a food bank to different satelite locations based on fairness and effectiveness.

The literature dealing with the nutritional aspects of food bank parcels and soup kitchen meals mostly aim to study the sufficiency of food provision to people in need. Eppich \& Fernandez (2004) compared the nutritional content of meals of a soup kitchen based in North Carolina, USA, with dietary reference intakes and daily reference values. They concluded that meals only fall short on some nutrients, even though the soup kitchen was providing three meals a day. Sprake et al. (2014) investigated the food intake of homeless people visiting a soup kitchen in Sheffield, UK, and concluded that the daily nutrient intake turned out to be significantly lower than the recommended intake. In a study on food parcels at a Dutch food bank, Neter et al. (2016) dealt with the nutritional value of parcels that completely consist of donated food. It became clear that the provided food was not meeting the nutritional standards. Total energy supply (in kJ) was sufficient, but the provided amount of fruit and fish was lower than recommended. Comparing the situation of a food bank to a soup kitchen, it should however be noted that food parcels are not necessarily supposed to cover the complete nutritional requirement of the beneficiaries, as the food parcels are often an addition to other sources of food supply. Furthermore, since food delivered by food banks is almost completely based on donated surplus food, it might also be hard to completely fulfil requirements. For soup kitchens, 
which can often buy additional ingredients to provide meals, the nutritional targets are potentially a more interesting benchmark.

\subsubsection{Research gap}

Even though donation management decisions are strongly impacted by menu planning decisions for a soup kitchen, and their menu planning is in turn heavily influenced by donation management, no research has combined these topics yet. Menu planning is only used in settings where food is not donated, and therefore not dealing with the extra complexity of short shelf life products and the menu planning limitations caused by donated products. Studies that do provide decision support for donation management are either vehicle routing or allocation problems, in which the decision to accept the donations is already made. However, whether a donation should be accepted depends on whether an ingredient can be used or not. This decision can be made by including menu planning. Menu planning models are widely available, using different mathematical techniques to solve the diet/menu problem. Research on soup kitchen clients or the soup kitchen meals shows that clients are highly dependent on the meals provided and can suffer from malnutrition. This raises the need to develop models that deal with food donations and can provide healthy meals to fulfil the clients' needs. In this chapter, we therefore address the benefits of arranging contracts with donors while providing meals to clients.

\subsection{Modelling approach}

A model is developed to answer tactical questions around the impact of different types of donation contracts. Therefore an optimization model is developed that integrates donation management and menu planning to answer questions such as (i) which (part of the) donations to accept, (ii) how to use adjust menu planning to use donations efficiently, (iii) how to deal with the complexity arising from food donations and menu planning, such as shelf life constraints and meal variety. In this section, we formulate the decision problem and the solution approach. First the notations of parameters and variables of the mathematical model will be explained, before we formalize the problem and discuss the rolling planning horizon approach. 


\subsubsection{Notation}

Sets and indices:

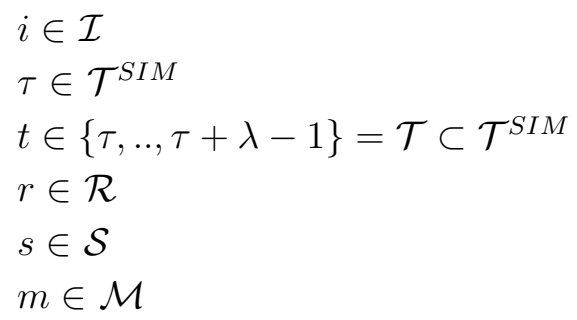

Ingredients

Time periods of full time horizon

Time periods in planning horizon

Recipes

Storage areas

Remaining shelf life

\section{Parameters:}

$c_{i}^{B} \quad$ Costs of ingredient $i$ per $\mathrm{kg}$ when bought $(€)$

$g \quad$ Discount given on buying price of ingredients when product is donated (proportion)

$c_{i}^{D} \quad$ Costs for ingredient $i$ per $\mathrm{kg}$ when donated, calculated by: $c_{i}^{D}=c_{i}^{B} *(1-g)(€)$

$c^{T} \quad$ Fixed transportation costs related to collection of donation $(€)$

$\lambda \quad$ Length of planning horizon (days)

$\chi \quad$ Number of periods it is prohibited to use an ingredient after it has been used (days)

$\omega \quad$ Number of periods it is prohibited to use a recipe after it has been used (days)

$\phi_{m} \quad$ Reward value for holding donation at end of planning period, based on remaining shelf life $m$

$m_{i} \quad$ Maximum shelf life of ingredient $i$ (days)

$\beta_{i m} \quad$ Binary value indicating that ingredient $i$ has shelf life $m$

$i_{i m}^{0} \quad$ Inventory of ingredient $i$ with remaining shelf life $m$ at beginning of planning period $(\mathrm{kg})$

vol $_{i} \quad$ Volume of ingredient $i$ in storage $\left(\mathrm{dm}^{3} / \mathrm{kg}\right)$

cap $_{s} \quad$ Capacity of storage $s\left(d m^{3}\right)$

$\alpha_{i s} \quad$ Binary value indicating that ingredient $i$ needs to be stored in storage area $s$

$q_{\text {ir }} \quad$ Quantity of ingredient $i$ needed for $1 \mathrm{~kg}$ of recipe $r(\mathrm{~kg})$

$u_{t i} \quad$ Binary value indicating that ingredient $i$ is used in period $t$

$v_{t r} \quad$ Binary value indicating that recipe $r$ is used in period $t$

$h \quad$ Minimum amount to make of a recipe when included in menu planning $(\mathrm{kg})$

$d_{t} \quad$ Meal demand in period $t(\mathrm{~kg})$

$a_{\text {tim }}$ Amount of ingredient $i$ offered in period $t$ with shelf life $m(\mathrm{~kg})$

$f_{\text {tim }} \quad$ Amount of ingredient $i$ donated under contract with shelf life $m$ to be collected in period $t(\mathrm{~kg})$

$x_{\text {tim }}$ Amount of ad hoc donation of ingredient $i$ with shelf life $m$ already accepted and to be collected in period $t(\mathrm{~kg})$

$t r_{t i} \quad$ Number of trips for collecting ingredient $i$ to make in period $t$

$M \quad$ Relatively large number 


\section{Donation management and menu planning}

Decision variables:

$I_{\text {tim }} \quad$ Inventory at start of period $t$ for ingredient $i$ with remaining shelf life $m(\mathrm{~kg})$

$X_{\text {tim }}$ Amount of ad hoc donation of ingredient $i$ with remaining shelf life $m$ to be collected in period $t(\mathrm{~kg})$

$B_{t i} \quad$ Amount of ingredient $i$ bought in period $t(\mathrm{~kg})$

$Y_{t r} \quad$ Amount of meal $r$ produced in period $t(\mathrm{~kg})$

$Z_{\text {tim }}$ Amount of ingredient $i$ used in period $t$ with remaining shelf life $m(\mathrm{~kg})$

$W_{t i} \quad$ Amount of ingredient $i$ wasted in period $t(\mathrm{~kg})$

$T R_{t i}$ Binary value with value 1 if donation of ingredient $i$ is collected in period $t$

$U_{t i} \quad$ Binary value with value 1 if ingredient $i$ is used in period $t$

$V_{t r} \quad$ Binary value with value 1 if recipe $r$ is used

\subsubsection{Problem and model description}

We consider a soup kitchen that provides $d_{t} \mathrm{~kg}$ of meals to clients once a day (based on current practice (Snels et al., 2012)). As only one meal is provided per day, it is not the goal to meet all nutritional recommendations, but by serving different meals throughout the planning horizon the soup kitchen can contribute to a healthy diet. The soup kitchen receives food donations mostly from parties such as warehouses, distribution centres, and retailers that are located in the same geographical area. Costs for collecting donations are thus almost identical, and are dominated by the (fixed) organizational effort of the soup kitchen to arrange the transport oneself (through volunteers) and the depreciation costs of the organization's vehicle(s), or by the fixed transportation costs a logistic service provider accounts. The volume of a donation usually fits well in a car or a mini-van. The transportation or collection costs of collecting a donation is fixed to $c^{T}$. In other settings a variable component can be added to the transportation costs, which we neglect here.

The planning horizon starts at day $\tau$, where a menu plan is developed for day $\tau$ until $\tau+\lambda$, based on current inventory levels $\left(i_{i m}^{0}\right)$ and available food donations $\left(a_{t i m}, x_{t i m}\right.$ and $\left.f_{\text {tim }}\right)$. To obtain a certain level of meal variety, menu planning considers restrictions on how often specific ingredients and recipes can be used. Therefore, the planning model keeps track of a tabu list of ingredients used in the last $\chi$ days and recipes used in the last $\omega$ days. For every day, decisions are made on the meals to serve, the ingredients to buy and the food donations to accept.

Donations occur ad hoc, and some are offered via contracts. For contractual donations, a soup kitchen is obliged to accept them, whereas the acceptance of ad hoc donations is the main donation management decision. Typically ad hoc donations become known only a few days in advance. Furthermore, ad hoc donations can be rejected or accepted in full 


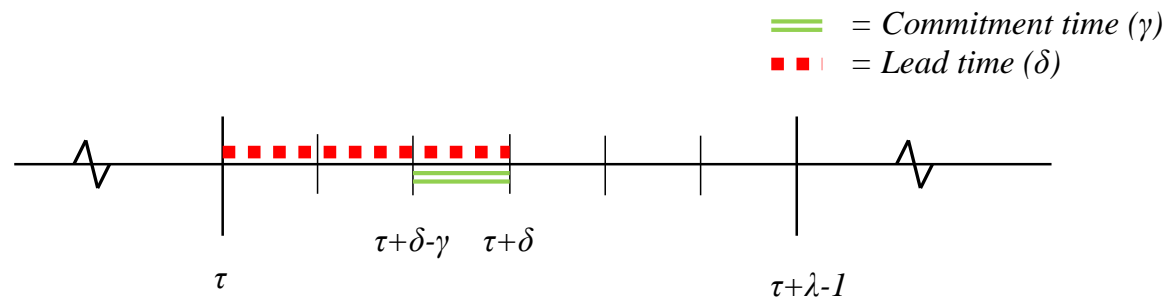

Figure 5.1: Planning horizon with length $\lambda$. At day $\tau$ ad hoc donations available to collect at $\tau+\delta$ are announced and the final decision on acceptance is made at $\tau+\delta-\gamma$.

or in part. Figure 5.1 shows the timing considerations related to ad hoc donations. The lead time $(\delta)$ is defined as the number of days between announcing/revealing information about the donation and the moment the donation is available to collect. The commitment time $(\gamma)$ is defined as the number of days between making a final decision on acceptance or rejection and the moment the donation is available to collect. This means that if we are at day $\tau$, the donation available at day $\tau+\delta$ will be announced. The soup kitchen has until $\tau+\delta-\gamma$ to decide to accept or reject this donation. When the donation is accepted in period $\tau+\delta-\gamma$, it must be collected in period $\tau+\delta$.

The focus of this chapter is on investigating tactical issues around contractual donations. A contract with the donor should allow for a more stable donation flow, better quality of the donated product and more useful donations. Therefore we differentiate the donations under contract from the ad hoc donations. We assume that the type of ingredients is fixed (vegetables, meat, or other ingredients) and the full information about the contract donation is revealed two days before the donation must be collected. Furthermore, we assume that the (average) shelf life of the ingredients donated via contracts is 1.5 times longer than the shelf life of the ingredients which are randomly donated, as there is a possibility of discussing shelf life within a contract.

\subsubsection{Rolling horizon}

The model is applied in a rolling horizon using simulation to capture the stochastic nature by which ad hoc donations are received. This approach allows us to include every day new donation information, and re-plan the menu for the remainder of the planning horizon. The full time horizon $\left(\mathcal{T}^{S I M}\right)$ comprises one simulated year, and the planning horizon is $\lambda$ periods (days). Figure 5.2 shows how the problem is solved in the rolling horizon. At the beginning of every period new information is revealed on the starting inventory levels $\left(i_{i m}^{0}\right)$, ad hoc donations are offered $\left(a_{t i m}\right)$ or need to be collected $\left(x_{\text {tim }}, f_{\text {tim }}\right)$, and the recipes $\left(v_{t r}\right)$ and ingredients $\left(u_{t i}\right)$ that were used in the previous periods (and limit decision making during the planning horizon). Note that donations are only known for 


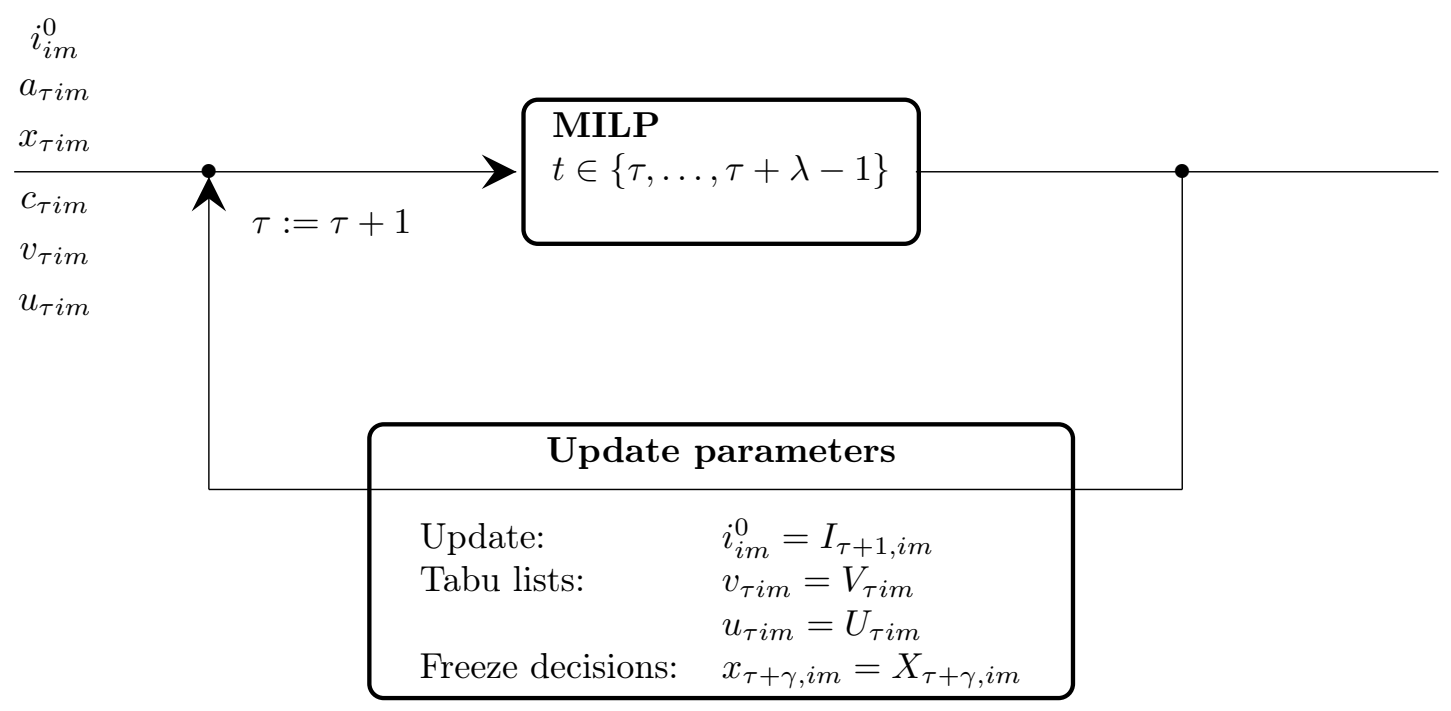

Figure 5.2: Rolling horizon algorithm: interactions between MILP and Simulation.

a part of the planning horizon meaning that donations available after $\tau+\delta$ are not yet considered. Then the planning horizon for all $t$, from $\tau$ until $\tau+\lambda-1$ is solved by the MILP model. After solving, we fix the menu for day $\tau$, update $i_{i m}^{0}$ by $I_{\tau+1, i m-1}, v_{\tau r}$ by $V_{\tau r}, u_{\tau i}$ by $U_{\tau i}$ and $x_{\tau+\gamma, i m}$ by $X_{\tau+\gamma, i m}$ and roll the planning horizon one day further to $\tau+1$ and plan again. As the model minimizes costs for the planning horizon, it does not consider the possibility to use donations arriving this planning period in a period beyond the planning horizon. To make sure those useful donations are still accepted, we give a reward towards holding inventory at the end of a planning period, based on the remaining shelf life of the ingredients, calculated by:

$$
\phi_{m}= \begin{cases}\frac{m-1}{\max _{i}\left\{m_{i}\right\}} & m<7 \\ 1 & m \geq 7\end{cases}
$$

In this formulation, the reward given to products increases with the remaining shelf life. Due to the variety constraints, ingredients with a longer shelf life have a higher probability to be used again before the remaining shelf life becomes zero.

\subsubsection{Mathematical optimization model}

The objective function (5.2) minimizes costs related to buying ingredients (5.3) and receiving and transporting donations (5.4) over the planning horizon $\lambda$. Remaining products in stock at the end of a period are given a positive value as they can be used in the next 
period and are therefore subtracted from the total costs (5.5).

$$
\text { Minimize } \sum_{t=\tau}^{\tau+\lambda-1}\left(B C_{t}+D C_{t}\right)-E I R
$$

subject to:

$$
\begin{aligned}
& B C_{t}=\sum_{i \in \mathcal{I}} B_{t i} \cdot c_{i}^{B} \quad \forall t \in \mathcal{T} \\
& D C_{t}=\sum_{i \in \mathcal{I}}\left(T R_{t i}+t r_{t i}\right) \cdot c^{T}+\sum_{i \in \mathcal{I}} \sum_{m \in \mathcal{M}}\left(X_{t i m}+f_{t i m}\right) \cdot c_{i}^{D} \quad \forall t \in \mathcal{T} \\
& E I R=\sum_{i \in \mathcal{I}} \sum_{m \in \mathcal{M}} I_{(\tau+\lambda) i m} \cdot c_{i}^{B} \cdot \phi_{m} \\
& \sum_{r \in \mathcal{R}} Y_{t r}=d_{t} \\
& \sum_{r \in \mathcal{R}} Y_{t r} \cdot q_{i r}=\sum_{m \in \mathcal{M}} Z_{t i m} \quad \forall t \in \mathcal{T}, i \in \mathcal{I} \\
& Y_{t r} \geq h \cdot V_{t r} \quad \forall t \in \mathcal{T}, i \in \mathcal{I} \\
& I_{\text {tim }}+\left(X_{\text {tim }}+f_{\text {tim }}+B_{t i} \cdot \beta_{\text {im }}\right)-Z_{\text {tim }}=\left\{\begin{array}{ll}
W_{t i} & , m=1 \\
I_{t+1, i, m-1} & , m \geq 2
\end{array} \forall t \in \mathcal{T}, i \in \mathcal{I}\right. \\
& \sum_{i \in \mathcal{I}} \sum_{m \in \mathcal{M}} I_{\text {tim }} \cdot \alpha_{i s} \cdot \operatorname{vol}_{i} \leq \operatorname{cap}_{s} \quad \forall t \in \mathcal{T}, s \in \mathcal{S} \\
& X_{\text {tim }}\left\{\begin{array}{ll}
=x_{\text {tim }} & \text {, if } t: \tau \leq t \leq \tau+\delta-\gamma \\
\leq a_{\text {tim }} & \text {,if } t: t+\delta-\gamma<t \leq t+\delta \\
=0 & \text {, else }
\end{array} \quad \forall t \in \mathcal{T}, \forall i \in \mathcal{I}, m \in \mathcal{M}\right. \\
& \sum_{m \in \mathcal{M}} X_{t i m} \geq M \cdot T R_{t i} \quad \forall t \in \mathcal{T}, i \in \mathcal{I}
\end{aligned}
$$




$$
\begin{array}{lc}
\sum_{m \in \mathcal{M}} Z_{t i m} \leq M \cdot U_{t i} & \forall t \in \mathcal{T}, i \in \mathcal{I} \\
\sum_{t^{\prime}=t-\chi-1}^{\tau-1} u_{t^{\prime} i}+\sum_{t^{\prime}=\max \{\tau, \tau-\chi-1\}}^{t} U_{t^{\prime} i} \leq 1 & \forall t \in \mathcal{T}, i \in \mathcal{I} \\
\sum_{r \in \mathcal{R}} Y_{t r} \leq M \cdot V_{t r} V_{t^{\prime}=t-\omega-1}^{\tau-1} v_{t^{\prime} r}+\sum_{t^{\prime}=\max \{\tau, \tau-\omega-1\}}^{t} \leq 1 & \forall t \in \mathcal{T}, r \in \mathcal{R} \\
I_{t i m}, I_{\tau+1, i m}, X_{t i m}, B_{t i}, Y_{t r}, Z_{t i m}, W_{t i} \in \mathbb{R}_{\geq 0} & \forall t \in \mathcal{T}, r \in \mathcal{R} \\
T R_{t i}, U_{t i}, V_{t r} \in\{0,1\} & \forall t \in \mathcal{T}, i \in \mathcal{I}, m \in \mathcal{M}, r \in \mathcal{R} \\
& \forall t \in \mathcal{T}, i \in \mathcal{I}, r \in \mathcal{R}
\end{array}
$$

Constraints (5.6) make sure that demand is met in every period. Constraints (5.7) make sure that enough ingredients are selected for the meal production. Constraints (5.8) ensure that when recipe $r$ is served, a minimum amount of $h \mathrm{~kg}$ is produced. Constraints (5.9) model unused inventory: unused ingredients with remaining shelf life 1 will go to waste, and all other ingredients become inventory for the next period. Constraints (5.10) ensure that inventory does not exceed the storage capacity at the soup kitchen. Constraints (5.11) and (5.12) deal with the available ad hoc donations. As ad hoc donations are only known by the soup kitchen until $\tau+\delta$ and the final decision has to be made at $\tau+\gamma$, no decision can be made for donations available to collect before $\tau+\gamma$. Furthermore, the accepted donations cannot be higher than the amount offered. When donations are accepted, they need to be collected (constraints (5.12)). Constraints $(5.13)-(5.16)$ deal with meal variety. When an ingredient $i$ is used, $U_{t i}$ will get value 1 in constraints (5.13). Constraints (5.14) subsequently ensure that an ingredient is only used once every $\chi$ days by checking the ingredients used during this planning period (stored in $\left.U_{t^{\prime} i}\right)$ and ingredients used in the previous period $\left(u_{t^{\prime} i}\right)$. Constraints $(5.15)$ and (5.16) work similarly, but apply to recipes instead of ingredients. The last constraints, (5.17) and (5.18), represent the variable domains. 


\subsection{Experimental design}

In this section, we formulate scenarios that allow us to analyse the benefits of structuring donations with suppliers via contracts, the influence of costs related to donations, the importance of timing aspects such as shelf life and moment of donation announcement and the costs related to meal variety. Table 5.1 provides an overview of the 25 experiments we study in this section.

\subsubsection{Scenarios}

Scenario Basic is used as a reference scenario, in which we do not consider any donations, such that all ingredients have to be bought. In the second scenario, $A H D$, we include ad hoc donations. Scenario Contract deals with receiving donations via contracts on top of the ad hoc donations. In experiments 3 to 11, we formulated 9 different contracts, varying in number of suppliers and donation moments (shown in Table 5.2). The acronyms, V1 to VMO5, indicate the type of product(s) involved and the frequency of donations. Donations either occur one, three, or five times per week. Suppliers are either delivering a box of vegetable products $(\mathrm{V})$, meat or fish products $(\mathrm{M})$, or other food products $(\mathrm{O})$. For example, with contract VM3, the soup kitchen receives 6 boxes per week: 3 times per week a donation of 1 box of vegetable (e.g. from a green grocery), and 1 box of meat/fish. The weight (in $\mathrm{kg}$ ) per box depends on the type of product.

The shelf life of donated ingredients can vary. Therefore, we also increase and decrease the remaining shelf life in scenario Shelf life. In the scenario Time, we vary the lead time of ad hoc donations $(\delta)$ and the commitment time $(\gamma)$. With a longer lead time, a soup kitchen knows earlier what will be donated by donors which potentially benefits planning. Changing the commitment time influences the flexibility in planning: a longer commitment time is expected to reduce flexibility.

The effects of the cost parameters are tested in scenario Transport cost and scenario Donation cost, in which we vary the transportation costs and the costs of donations, in cases where less than $100 \%$ discount is given. This allows us to evaluate benefits or drawbacks of different cost structures.

In the last scenario (scenario Meal variety), we test how the constraints on ingredient and recipe use affect the donation use, costs, and menu selection of the soup kitchen. 
Table 5.1: Design of experiments.

\begin{tabular}{|c|c|c|c|c|c|c|c|c|}
\hline \# & Scenario & Contract & Shelf life & $\gamma / \delta$ (days) & $g$ & $c^{T}(€)$ & $\omega$ (days) & $\chi$ (days) \\
\hline 1 & Basic & - & - & - & - & - & 7 & 2 \\
\hline 2 & $A H D$ & - & 2 days & $2 / 1$ & 1 & 3.50 & 7 & 2 \\
\hline 3 & Contract & V1 & 2 days & $2 / 1$ & 1 & 3.50 & 7 & 2 \\
\hline 4 & & $\mathrm{~V} 3$ & & & & & & \\
\hline 5 & & V5 & & & & & & \\
\hline 6 & & VM1 & & & & & & \\
\hline 7 & & VM3 & & & & & & \\
\hline 8 & & VM5 & & & & & & \\
\hline 9 & & VMO1 & & & & & & \\
\hline 10 & & VMO3 & & & & & & \\
\hline 11 & & VMO5 & & & & & & \\
\hline 12 & Shelf life & VM3 & 1 day & $2 / 1$ & 1 & 3.50 & 7 & 2 \\
\hline 13 & & & $0.3 * m_{i}$ & & & & & \\
\hline 14 & Time & VM3 & 2 days & $1 / 1$ & 1 & 3.50 & 7 & 2 \\
\hline 15 & & & & $2 / 2$ & & & & \\
\hline 16 & & & & $7 / 1$ & & & & \\
\hline 17 & & & & $7 / 2$ & & & & \\
\hline 18 & Donation cost & VM3 & 2 days & $2 / 1$ & 0.2 & 3.50 & 7 & 2 \\
\hline 19 & & & & & 0.4 & & & \\
\hline 20 & & & & & 0.6 & & & \\
\hline 21 & Transport cost & VM3 & 2 days & $2 / 1$ & 1 & 0.00 & 7 & 2 \\
\hline 22 & & & & & & 7.00 & & \\
\hline 23 & Meal variety & VM3 & 2 days & $2 / 1$ & 1 & 3.5 & 1 & 2 \\
\hline 24 & & & & & & & 4 & 2 \\
\hline 25 & & & & & & & 7 & 1 \\
\hline
\end{tabular}

Table 5.2: List of contracts.

\begin{tabular}{l|lll}
\hline Contract & Product category & Donation days & Boxes/week \\
\hline V1 & Vegetable & 1 & 1 \\
V3 & Vegetable & 3 & 3 \\
V5 & Vegetable & 5 & 5 \\
VM1 & Vegetable, Meat/Fish & 1 & 2 \\
VM3 & Vegetable, Meat/Fish & 3 & 6 \\
VM5 & Vegetable, Meat/Fish & 5 & 10 \\
VMO1 & Vegetable, Meat/Fish, Other & 1 & 3 \\
VMO3 & Vegetable, Meat/Fish, Other & 3 & 9 \\
VMO5 & Vegetable, Meat/Fish, Other & 5 & 15 \\
\hline
\end{tabular}

\subsubsection{Parameter settings and assumptions}

The model deals with 40 ingredients $(\mathcal{I})$, which can be combined into 89 unique recipes $(\mathcal{R})$, based on a study at the Salvation Army in the Netherlands (Snels et al., 2012). 
Recipes only consider the main ingredients, minor ingredients such as salt and spices are neglected. 16 ingredients have a maximum shelf life $\left(m_{i}\right)$ of 22 days, 10 ingredients of 14 days and the other ingredients 7 days or less. Ingredients are either stored frozen $(s=1)$, refrigerated $(s=2)$, or ambient $(s=3)$. Demand $\left(d_{t}\right)$ is assumed to be constant and deterministic $\left(30 \mathrm{~kg} /\right.$ day), and the initial inventory level $\left(i_{i m}^{0}\right)$ is assumed to be zero. Initially, every recipe can be used only once in seven days $(\omega=7)$, whereas ingredients can be used every other day $(\chi=2)$. Ingredient costs $c_{i}^{B}$ are retail prices of a Dutch retailer. Fixed costs related to buying ingredients are neglected, as are labour costs. Fixed transport costs of $€ 3.50$ are in current when collecting a donation.

To obtain a data set for ad hoc donations we used information received from a Dutch food bank. From this, we created a dataset with donations $\left(a_{t i m}\right)$ for the full simulation horizon $\left(\mathcal{T}^{\mathcal{S I M}}\right)$, specifying the ingredient and quantity. The total quantity of the donations are

set to be $\frac{3}{7}$ of the total demand (Neter et al., 2016). In our experiment, food donations contain ingredients that are randomly selected from our ingredient list, where ingredients present in the data received from the food bank have a higher chance to get selected. Donation quantities are based on the weight of a full box of the ingredient (and thus vary per ingredient) and the number of boxes donated is determined by a binomial distribution: $1+\operatorname{Bin}(n, p)$ with $n=3$ and $p=0.2$. Donation data for contract donations $\left(f_{\text {tim }}\right)$ is also generated for the full simulation horizon $\left(\mathcal{T}^{\mathcal{S I M}}\right)$, where the ingredients are randomly picked.

The problem is solved with a rolling horizon approach for 1 year $\left(\mathcal{T}^{\mathcal{S I M}}\right)$. Within the rolling horizon approach, each planning problem has a planning horizon of 7 days $(\lambda)$ and is thus solved 358 times for each experiment. To deal with variation in donations, 10 versions of the datasets $a_{\text {tim }}$ and $f_{\text {tim }}$ are created. Experiment 2 is executed 10 times with all datasets for $a_{\text {tim }}$, and experiments 3-25 are carried out with 10 versions of dataset $f_{\text {tim }}$. For each experiment, the MILP is thus solved $358 * 10$ times. The model is implemented in Xpress-IVE 7.9 and solved using the Fico Xpress Optimizer. It takes about 1.5 seconds to solve the MILP for one day on a PC with Intel Core i5-5300U CPU $2.3 \mathrm{GHz}$.

\subsection{Numerical results}

Evaluation of the scenarios is based on four performance measurements. First, costs are incurred for buying ingredients, transporting donations, and in some cases for buying donations with discounts. The model minimizes total costs over a full planning horizon, which also includes periods for which the planning can still be adjusted. Therefore, the reported costs are obtained by summing up all costs made in the first period of every 
planning horizon. This ensures we only report the actually incurred costs. Second, we measure meal variety. This measure shows how often recipes are used during the full time horizon. Third, we measure the donation acceptance rate for the ad hoc donations, since we want to evaluate if there are options to increase this rate, and more surplus food can be used. Finally, we measure food waste obtained at the soup kitchen, since donated food might not always be used. Results presented in the following sections are average values over 10 runs, with standard deviations of $1 \%$ or less for each experiment.

\subsubsection{Number of donations and contracts}

Figures 5.3 to 5.6 show the main results for the different performance measures for the scenarios Basic, AHD and Contract. These results mainly provide insights in the interaction between ad hoc donations and contract donations based on the number of donations and the contract types.

In scenario Basic, where no donations are offered, total costs are the highest, indicating that any kind of food donation reduces overall costs (Figure 5.3). Cost reductions lie between $13.9 \%$ for contract $V 1$ and $22.6 \%$ for contract VM5 compared to scenario Basic. Results show furthermore that the share of transportation costs increases with an increase in donations. However, an increase in contract donations does not necessarily result in lower overall costs, especially when transportation is relatively expensive compared to the buying prices. For instance, the ingredient price of potatoes is $€ 1.00$ per $\mathrm{kg}$, whereas transporting a donation is $€ 3.50$. As ingredient prices differ per product, the trade-off between buying an ingredient or collecting it at a donor is different for each product. For contracts VM1-VM5, the reduction in buying costs is larger (in number and percentages) than for contracts $V 1-V 5$ as more expensive products are donated.

Figure 5.4 shows how often recipes are selected as a percentage of the total meal production over the full horizon. Out of 89 possible recipes, we can for instance see that scenario Basic only uses 13 recipes, of which 7 are used $4-6 \%$ of the time and 6 recipes $12-14 \%$ of the time. This illustrates the variety constraints leading to a minimum level of variety in meals served. Any situation in which donations are offered has significantly more meal variety. For all donation scenarios, most recipes are used only a few times, and none of them more than $8 \%$ of the time. Furthermore, the number of recipes that are not selected decreases significantly, from 76 in scenario Basic to an average of 6.4 in the donation scenarios, and as low as zero in the scenario where the most donations are offered (VMO5).

The percentage of accepted donations changes if more donations are offered via contracts, even though this is a minor change (see Figure 5.5). In scenario AHD, $45 \%$ of the total 


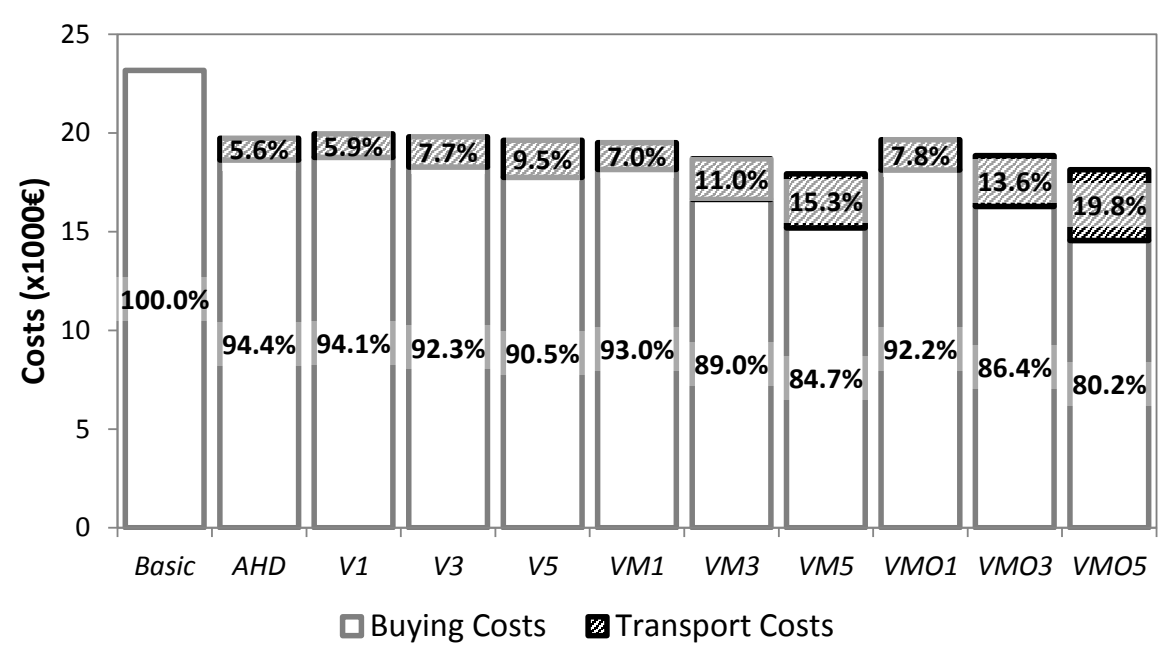

Figure 5.3: Total costs for scenario Basic, AHD and Contract divided per cost contribution. Percentages are related to total costs.

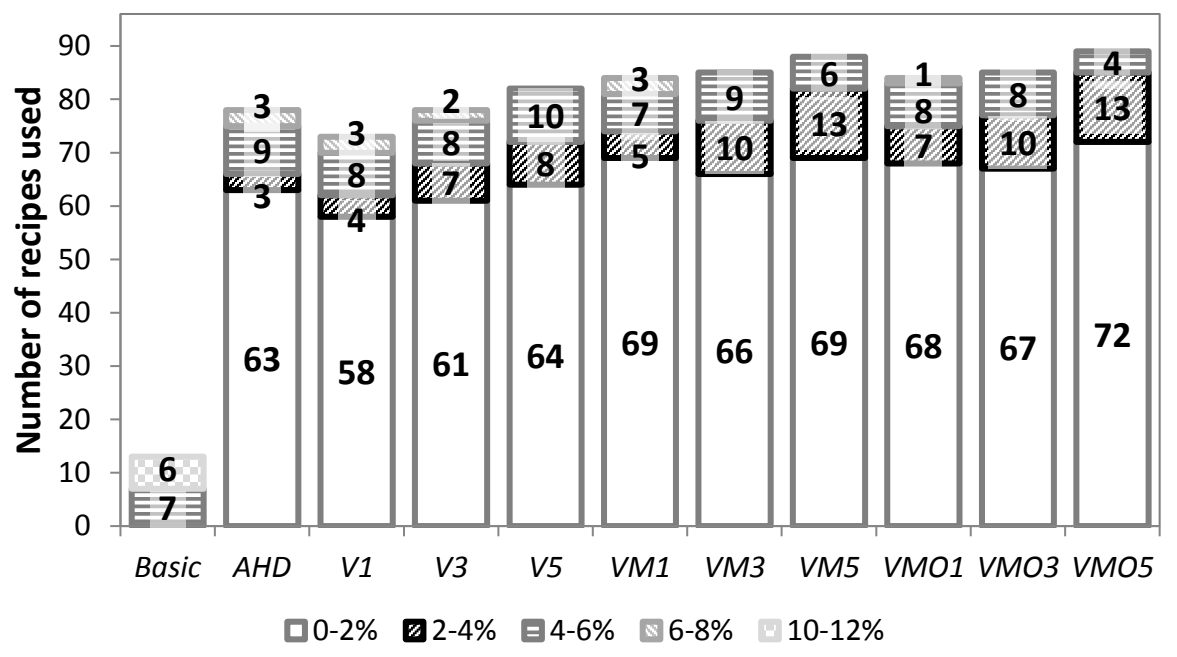

Figure 5.4: Menu selection for scenario Basic, AHD and Contract. The numbers above indicate total number of recipes selected.

amount of offered donations is accepted (in $\mathrm{kg}$ ); as donations can be accepted partly this amount is obtained from $61 \%$ of the number of donations offered. If donations are not useful, the soup kitchen will choose not to accept them and avoid the transportation costs resulting from collection. The decrease in donation acceptance, if using contracts compared to only ad hoc donations is relatively small. When contract donations are easy to combine with the ad hoc donations in a menu plan, more donations can be used.

Even though the acceptance rate did not show large changes, when more donations arrive at the soup kitchen, food waste at the soup kitchen does increase significantly (as shown in Figure 5.6). Most donations ending in food waste at the soup kitchen are received via a 


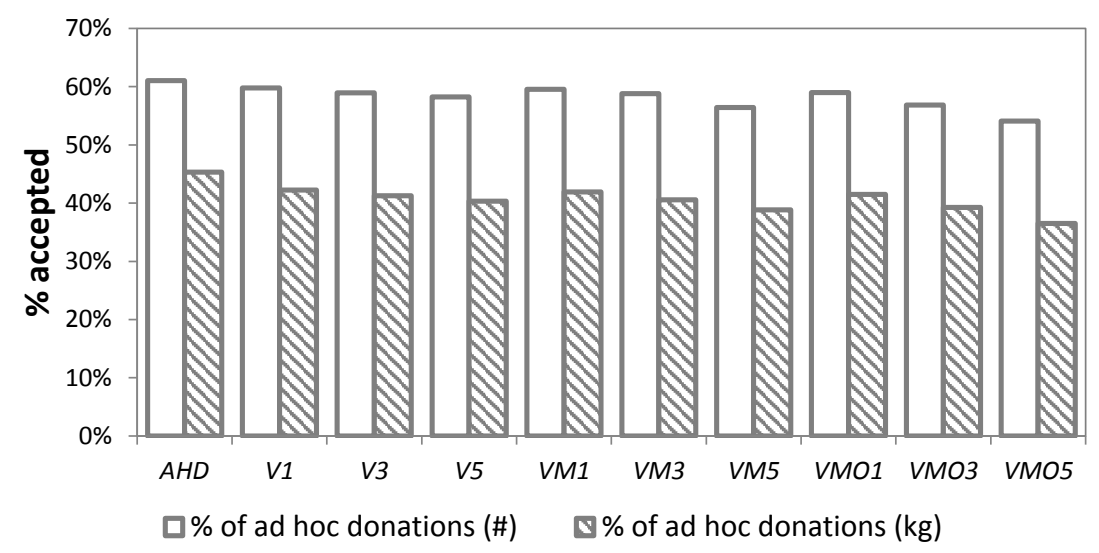

Figure 5.5: Acceptance in $\mathrm{kg}$ and number of offered donation for scenario AHD and Contract.

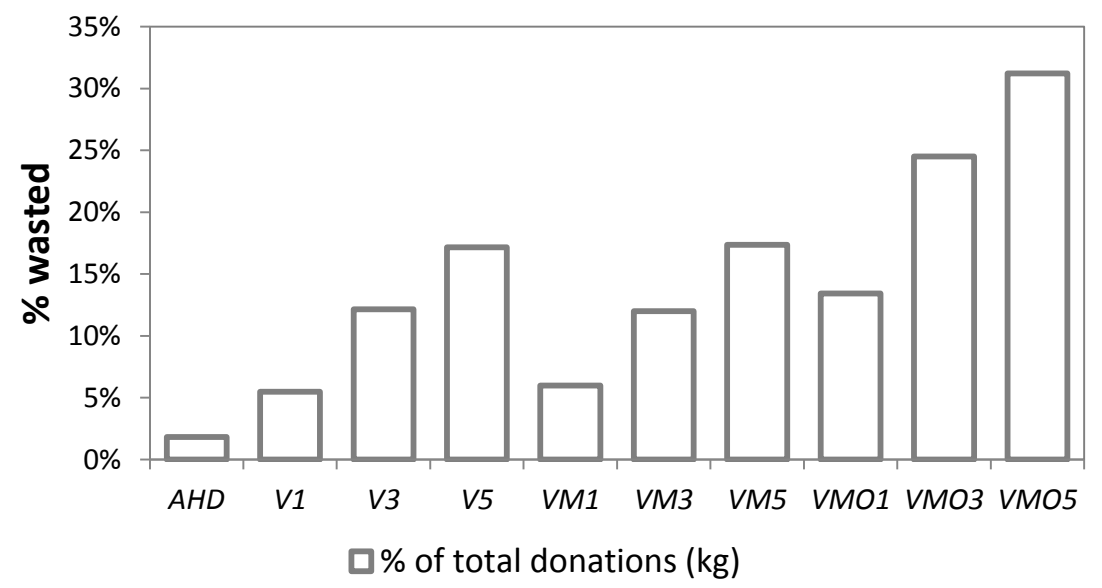

Figure 5.6: Food waste in $\mathrm{kg}$ of incoming donations for scenario AHD and Contract.

contract as a soup kitchen will not accept ad hoc donations that cannot be used. Making a meal out of donated ingredients usually requires additional ingredients to be bought. When it is then cheaper to make meals that completely consist of bought ingredients, a soup kitchen will waste donated ingredients. However, it is not only the contract donations that end up as waste; some of the accepted ad hoc donations can end up as waste as well. The soup kitchen must decide $\gamma$ days before collection whether a donation is accepted or not, but the menu planning can still change afterwards based on cost savings resulting from new donation information.

\subsubsection{Shelf life of donations}

To evaluate the effect of changes in the remaining shelf life of products donated, we study three alternatives: the 2 days from the basic scenario, an increase to $30 \%$ of the maximum shelf life of the product $m_{i}$, and a decrease to 1 day (note that shelf life of 

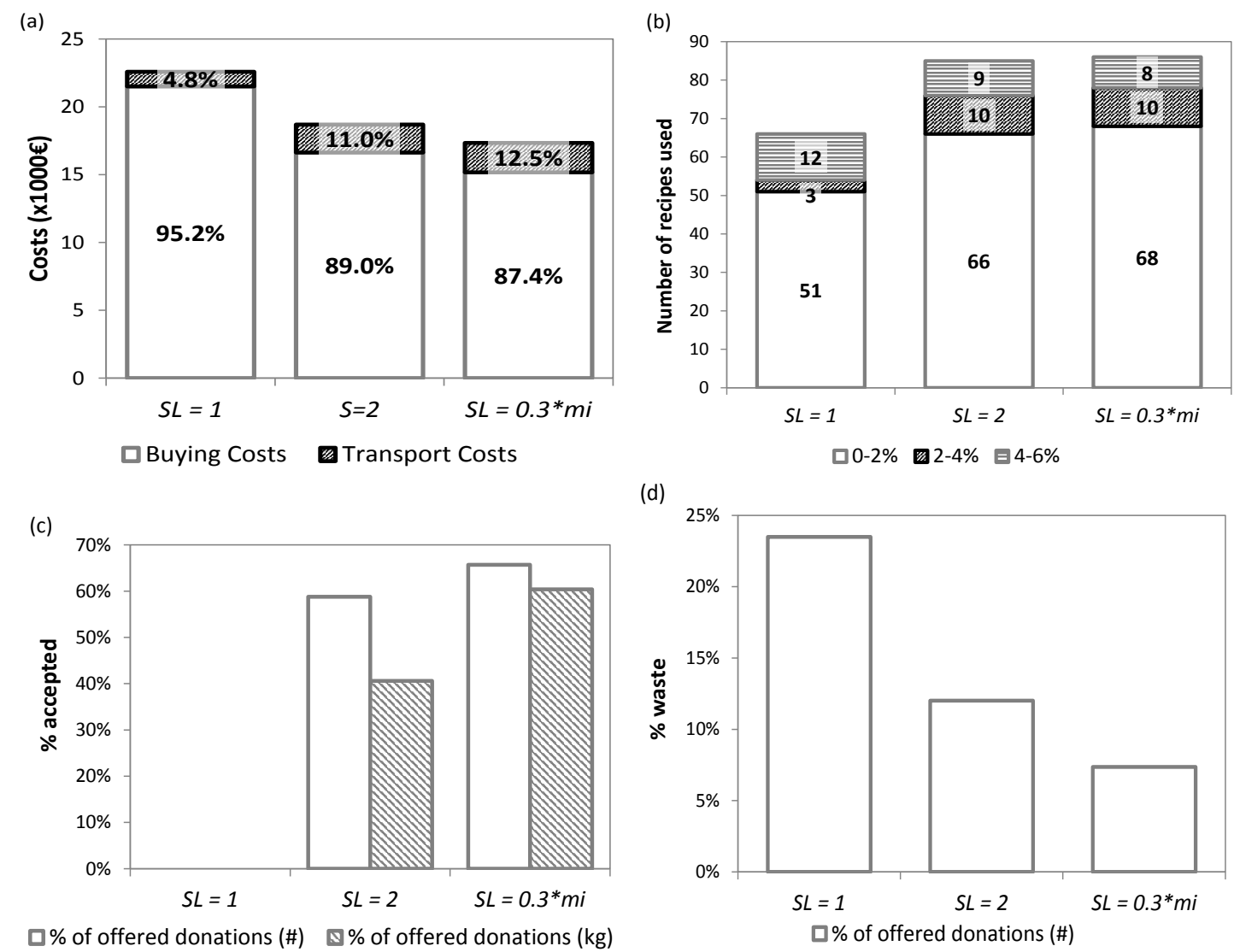

Figure 5.7: Results of scenario Shelf life (a) Total costs, (b) Menu selection, (c) Donation acceptance, (d) Waste.

contract donations are 1.5 times longer). Figure 5.7(a) shows that, when shelf life is increased, total costs decrease and donation acceptance increases because products can be used for a longer time. The decrease in costs is thus achieved by a decrease in buying costs, since more transportation costs are incurred due to the higher acceptance rate. Total costs increase to almost the level of scenario Basic when ad hoc donations only have a shelf life of 1 day. Here, many donations cannot be used efficiently in menu planning and are therefore rejected (Figure 5.7(c)), leaving the soup kitchen with only contract donations and a significant decrease in meal variety (as reflected in the recipe use shown in Figure 5.7(b)). Figure 5.7(c) shows that the percentage of accepted ingredients in $\mathrm{kg}$ of ingredient offered and the percentage of accepted ingredients in units of offered donations deviate less at a higher remaining shelf life. As there is more time to use the ingredients, larger volumes can be accepted and used. Waste levels shown in Figure 5.7(d) are in line with the expectations: when there is more time to use the donations, waste levels will decrease. 


\section{Donation management and menu planning}

\subsubsection{Lead time of donations}

The moment ad hoc donations are announced or the time there is to decide to accept or reject a donation can vary. Therefore, we study five cases, in which the lead time varies between 1 or 7 days, and the commitment time is 1 or 2 days for ad hoc donations. Figure $5.8(\mathrm{a}, \mathrm{b})$ show that different lead times or different commitment times have a relatively small impact on costs and meal variety, although there are differences in donation acceptance rates and waste. When commitment time $(\gamma)$ increases with the same lead time $(\delta)$, less donations are accepted and waste obtained increases (Figure 5.8(c,d)). When decisions must be made longer in advance, there is less information available on upcoming donations. The optimal menu plan can change more easily after making the decision on accepting the donation when more useful donations will be available later. Therefore, the highest donation acceptance rate and the lowest amount of waste is obtained when lead time $(\delta)$ is 2 days and commitment time $(\gamma)$ is 1 day.

\subsubsection{Donation cost and transport cost}

In this section, we study the influence of the main cost factors related to donation management. In scenario Donation cost, we study the influence of receiving a discount on food ingredients instead of receiving free food donations. In scenario Transport cost, we study the influence of transportation costs. As expected, Figure 5.9(a) clearly shows that introducing a donation cost leads to an increase in total costs. Furthermore, the meal variety decreases when costs increase (Figure 5.9(b)). Also, Figure 5.9(c) shows that donation acceptance decreases with higher costs. When donations costs increase, the trade-off between collecting an ingredient as a donation or buying it fresh at the retailers changes. For a decreasing number of ingredients, it will be beneficial to obtain them as a donation. Figure 5.9(d) shows a small variation in waste, however this is negligible as it is caused by the stochastic nature of the problem.

Transportation costs have similar effects as costs for donations, as shown in Figure 5.10. Even when there are no transportation costs (e.g. the donor brings the donation), part of the donations is still not accepted (Figure 5.10(c)). This shows that only donations that can be used by the soup kitchen are accepted. Analysing the details of donation acceptance decisions show that at most the needed amount for a day is accepted, due to the variety constraints, the total demand, and the limited shelf life. If a larger quantity is offered, the donations will only be partly accepted. Donations are completely rejected if the ingredient cannot be used the next day due to variety constraints. The meal variety shown in Figure 5.10(b) shows the expected trend: more accepted donations will result in a higher number of recipes used throughout the simulation horizon. In line with 

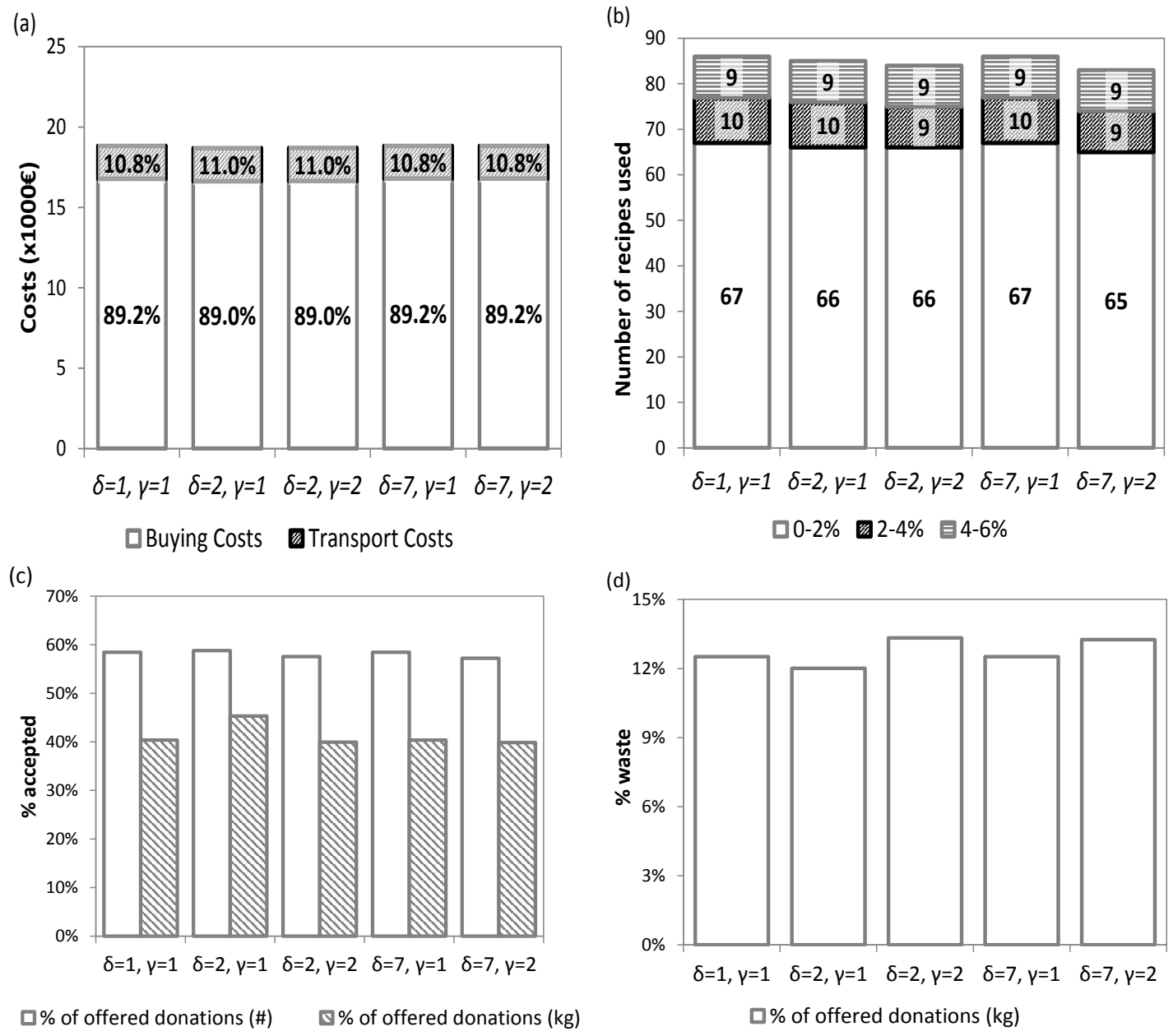

Figure 5.8: Results of scenario Time (a) Total costs, (b) Menu selection, (c) Donation acceptance, (d) Waste.

expectations, the differences in waste obtained (Figure 5.10(d)) are small for scenario Donation cost. If donations are more expensive, less donations will be accepted and therefore waste will decrease slightly. When transportation costs change, waste levels do not change, although more food donations are accepted. However, it still is not useful to accept donations which cannot be used.

\subsubsection{Meal variety}

In the previous scenarios a restriction on the use of ingredients and recipes was used. In the last scenario, these bounds are relaxed. Figure 5.11 shows the results of a relaxation of the recipe bound. When recipes can be used every day $(\omega=1)$ total costs are reduced significantly (Figure 5.11(a)). This reduction is obtained by an increase in the use of the recipe with the lowest cost. As shown in Figure 5.11(b), there are two recipes used 
(a)

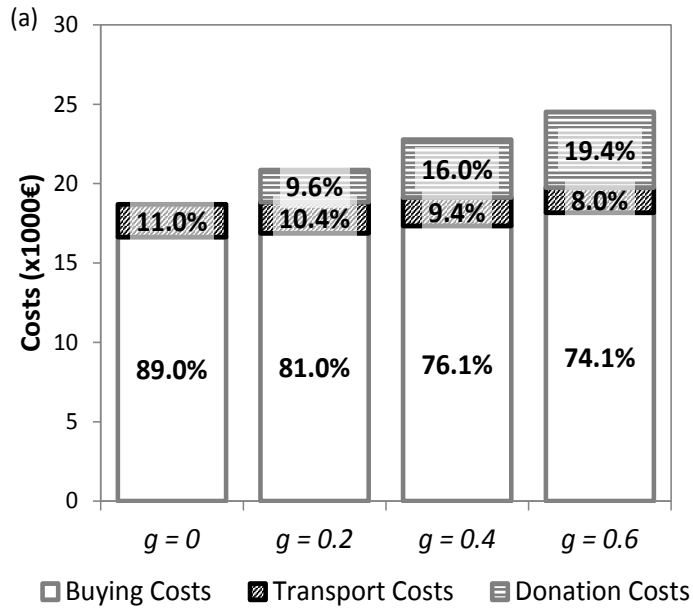

(c)

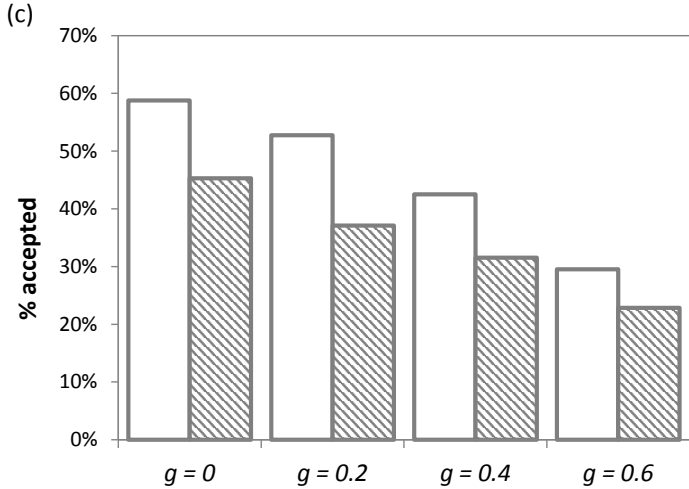

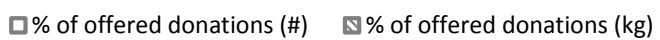

(b)

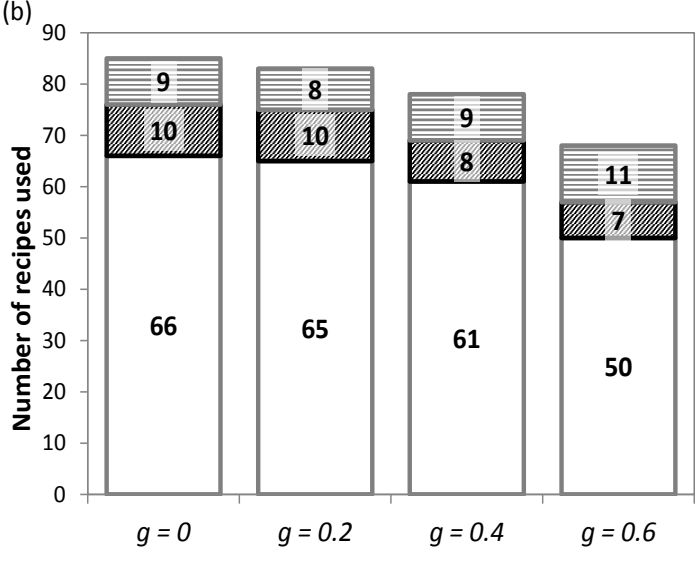

(d)

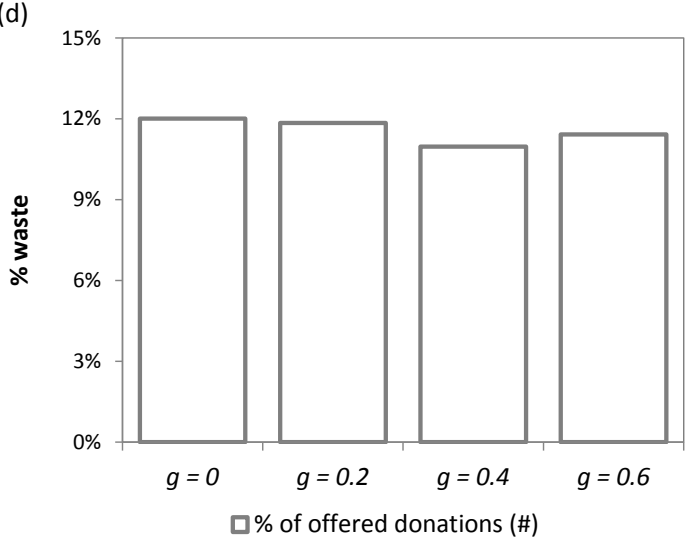

Figure 5.9: Results of scenario Donation cost (a) Total costs, (b) Menu selection, (c) Donation acceptance, (d) Waste.

more than $18 \%$ of the time. Furthermore, the donation acceptance is reduced, and a large decrease in waste levels is obtained, as shown in Figure 5.11(c,d). When the restriction on recipes is maintained, but reduced to 4 (i.e. allowing the same recipe to be used every four days), costs still decrease compared to the restriction of 7 days, but the meal variety is not affected. However, the waste levels decrease from $12 \%$ to $3 \%$.

When the recipe restriction is maintained, but the ingredient restriction is relaxed, similar results are obtained. Figure 5.12 (a) shows a small decrease in costs, meal variety, and donation acceptance when ingredients can be used every day (see Figure 5.12(b,c)). However, as shown by Figure 5.12 (d), waste levels are reduced significantly.

\subsection{Decision rules for accepting ad hoc donations}

Donation acceptance decisions are difficult to formalize in practice. Decisions on acceptance of fresh produce are between accepting all (to prevent a loss of goodwill by donors), 

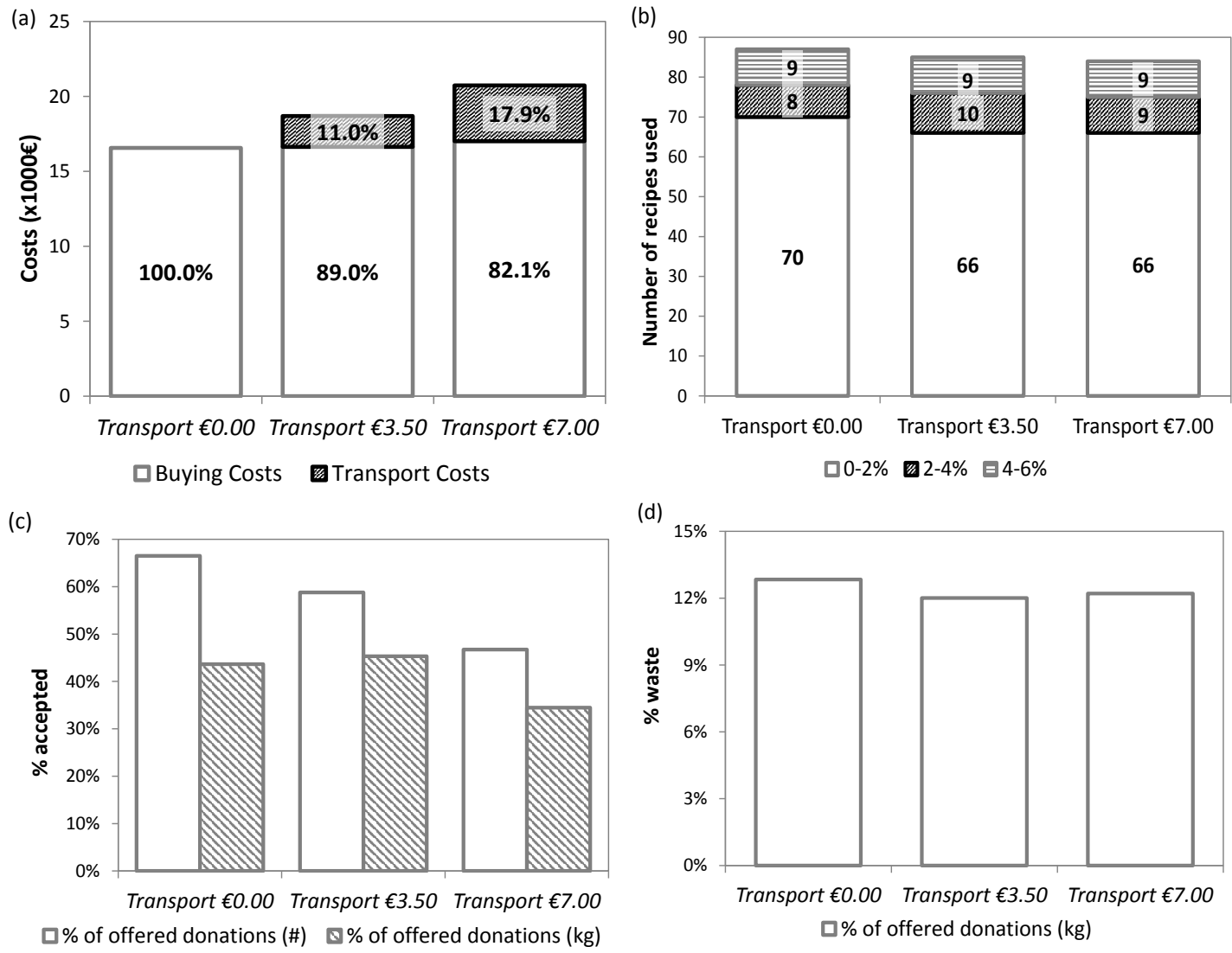

Figure 5.10: Results of scenario Transport cost (a) Total costs, (b) Menu selection, (c) Donation acceptance, (d) Waste.

and accepting not too much of each ingredient to enforce meal variety, to meet shelf life constraints, and to reduce waste at soup kitchens. Currently one tends to accept as much as possible and leave the menu planning and prevention of waste to the chef's creativity. If a donation does not fit in the menu plan, the donation is rejected or the menu is adjusted such that the donation can be used. The MILP model includes both options and thus fits to current practice. In case one cannot use a donation in full, in practice the donation may be redirected to other organisations. The scope of this chapter is to assess the value of donation contracts for a single organisation, thus redirection of donations is beyond the scope. In this section, the performance of the MILP model is compared against the following decision rules that relate to the above considerations on accepting ad hoc donations:

1. All $=$ Accept the full volume of all donations,

2. All-day $=$ Accept all donations, volume limited to quantity of one day,

3. VMO-day = Accept per category (vegetable (V), meat or fish $(\mathrm{M})$, or other food products $(\mathrm{O})$ ) one donation and volume limited to quantity of one day. 

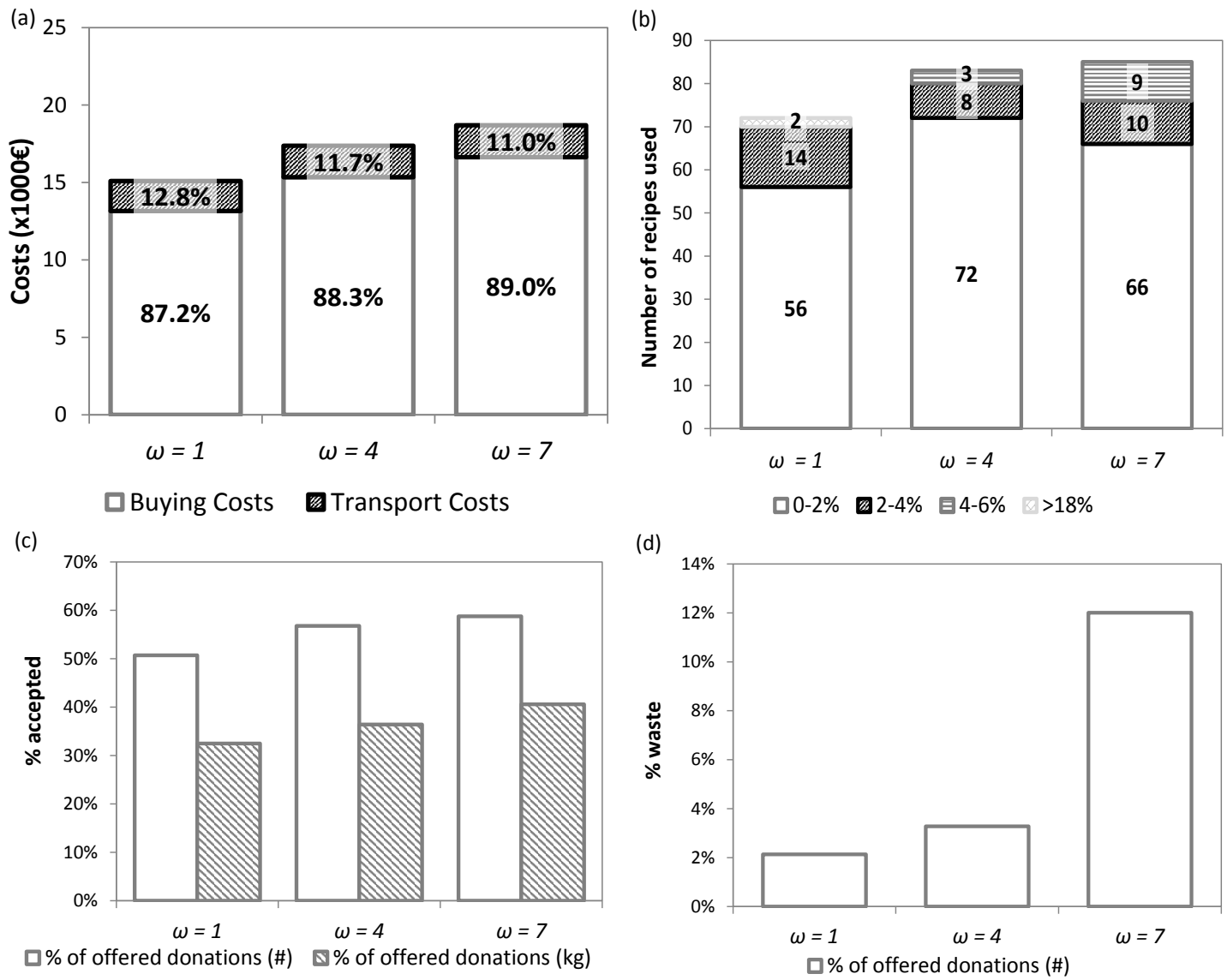

Figure 5.11: Results of scenario Meal Variety with different $\omega$ (a) Total costs, (b) Menu selection, (c) Donation acceptance, (d) Waste.

The idea of limiting the volume to one day in rules All-day and VMO-day, is triggered by the results for MILP and by the meal and ingredient variety constraints and the short remaining shelf life of donated products. Note that these rules are easy to apply in practice, but leave the menu planning and waste reduction to the chef. To evaluate these decision rules, the MILP model is used by restricting the values of the donation acceptance variables $\left(X_{\text {tim }}\right)$. The restricted MILP model determines a cost-optimal menu planning that makes good use of the accepted donated, and thereby approximates the non-formalized decision of the chef. The results are reported in Figure 5.13.

The results of scenario $A H D$ are cost-optimal solutions from the previous section. Accepting all ingredients leads to an overall cost increase of $2.6 \%$, which is mostly due to a $60 \%$ increase in transportation costs, and a very high percentage of food waste at the soup kitchen. In practice, such a rule is only sustainable if excessive donations are redirected to and used by other organisations, but for products with short shelf lives this might not be possible. Although the cost increase is only $2.6 \%$, this is still an unwanted increase for the soup kitchen, as they are cost driven. Rule All-day still accepts all donations but 

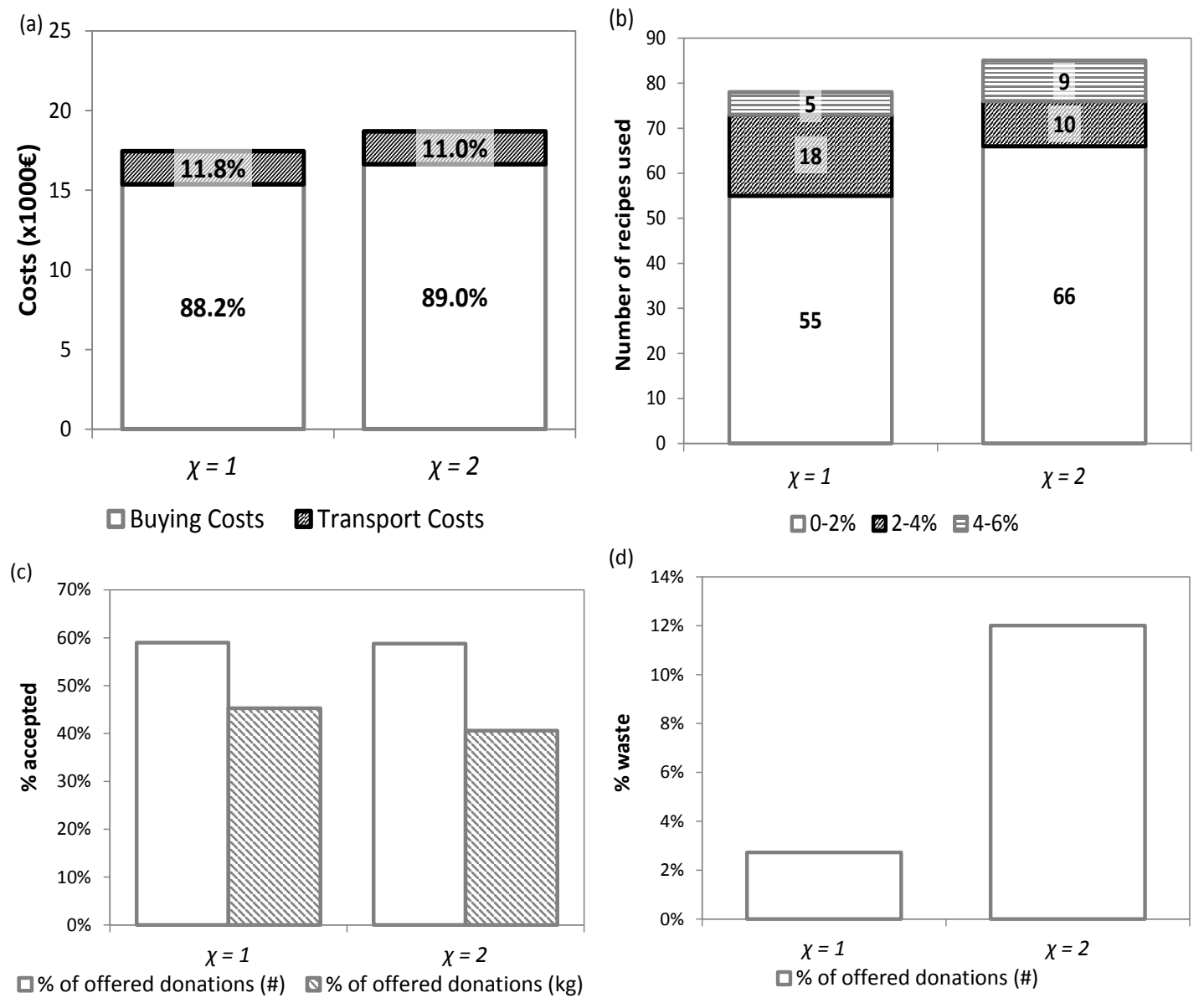

Figure 5.12: Results of scenario Meal Variety with different $\chi$ (a) Total costs, (b) Menu selection, (c) Donation acceptance, (d) Waste.

limits the accepted quantity to the need for a single day. This reduces waste from $19.1 \%$ to $7.8 \%$, but some donations do still not fit into a cost-optimal meal plan. Note that even in a cost optimal plan (scenario $A H D$ ), waste is still $1.8 \%$ as some accepted donations become redundant by more favourable donations that occur later. Furthermore a cost increase of $2.5 \%$ is obtained for the All-day rule, compared to scenario $A H D$. Finally, the rule VMO-day yields lower waste but higher costs $(+1.9 \%)$ by accepting only one donation per category per day. The overall acceptance rate is slightly lower than scenario $A H D$. Besides higher costs, this rule results in a lower meal variety: 72 vs 78 different recipes are used.

Our results (and decision rules) are relevant for products with relatively short shelf lives. The MILP can easily deal with longer shelf lives (as shown in Section 5.5.2). However, the decision rules have to become more complex to better assess the value of products with a (much) longer shelf life, i.e. detailed stock keeping administration and meal planning decisions should be included at some level. Both are included in the MILP, which makes 

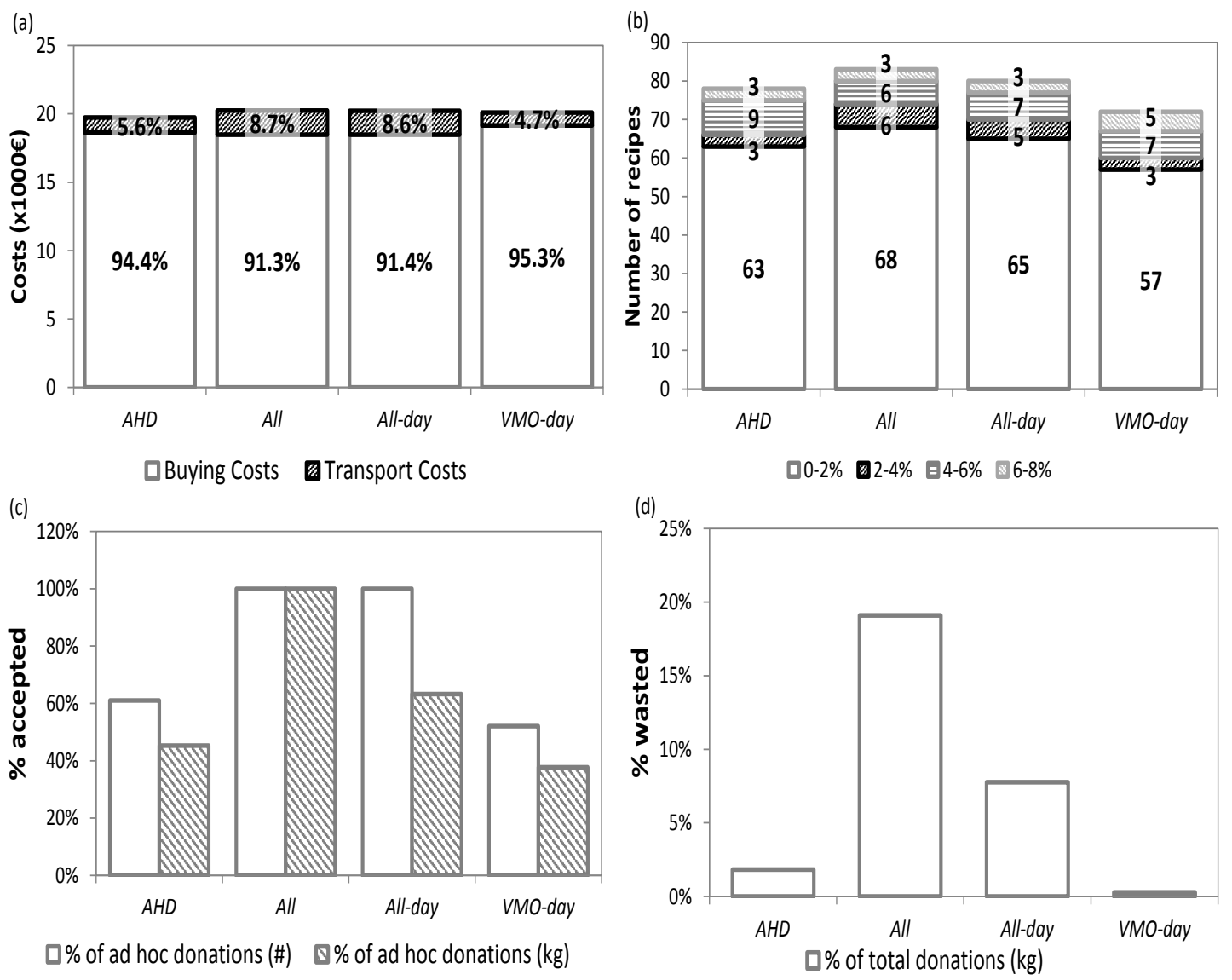

Figure 5.13: MILP model vs decision rules (a) Total costs, (b) Menu selection, (c) Donation acceptance, (d) Waste.

the model promising for operational use next to analysing tactical issues.

\subsection{Conclusion and discussion}

In this chapter, we combine donation management and menu planning for a soup kitchen, in order to efficiently use food donations and reduce food waste. This integrated planning problem has so far not been addressed in the literature. Menu planning is a planning problem dealing with the selection of recipes, and obviously interacts with decisions on whether donations should be accepted or not. Depending on (variety) constraints introduced in the menu planning model, only a certain share of the offered donations can actually be used efficiently.

International developments regarding food waste reduction (e.g. recently introduced French legislation forcing retailers to donate food surplus to charitable organizations) make it interesting and relevant to not only study donation management, but also to in- 
vestigate the effect of receiving donations via contracts. Our results show that contracts are a good addition to ad hoc donations, but also show that not all donations arriving at soup kitchens can be used, despite their consideration in menu planning. Costs related to donations, such as transportation or purchasing costs, decrease the attractiveness of donations for soup kitchens. However, one of the benefits of setting up contracts is the possibility for agreements on frequency, quantity, remaining shelf life, and type of ingredient, which all influence the usefulness of donations. Furthermore, restrictions to ensure meal variety are indeed useful in order to serve the clients a varied and arguably healthier meal throughout the week, even though a higher meal variety cause higher waste levels. From a palatability point of view, ingredient or recipe variety is also preferred, but to what extent this is worth the additional food waste and increased costs is an important managerial discussion that gets input from the results in this chapter.

In this chapter, we focus on ingredient purchasing and transportation costs. Labour costs are often no economic costs to charitable organizations, since they tend to work with volunteers. Other costs (e.g. overhead costs, meal production costs) are present, and will impact the overall costs, but these costs are not expected to have a significant impact on the trade-offs in the integrated donation management and menu planning problem we considered in this chapter.

Furthermore it is assumed that ingredients donated via contracts do have a longer shelf life, as there is a possibility to include a minimum on the shelf life of ingredients within a contract. When the shelf life of contract donations would be similar to the ad hoc donations, the number of donations that can be used will decrease, thus less ad hoc donations will be accepted.

The exclusion of storage capacity and collection truck capacity might influence results if the model is applied to products with a long shelf life. However, if donation quantities are in line with the size of the soup kitchen, the influence of storage capacity is expected to be small. Whether a truck capacity should be included depends on how transportation is organized. Many logistic service providers imply a fixed costs for collecting and delivering products within a certain geographical area. If a (group of) soup kitchen(s) is organizing the transport them selves either (fixed) costs apply for compensating volunteers, or these costs are to be shared if multiple donations are combined in a single trip. In the later case then the collection truck capacity might influence the results, and it is therefore interesting for further research to combine the work in this chapter with research on vehicle routing for charitable organisations (see Section 5.2). This allows the intelligent consideration of truck capacities, both for collecting donations as well as purchasing fresh ingredients. 
The proposed optimization model is used in this chapter for evaluation on the tactical level. However, soup kitchens could also benefit from such decision support systems to help them make operational decisions. However, in order to implement the developed model in such a way, there would be investments in the soup kitchen's IT infrastructure and data management. Charitable organisations usually lack the time and money to fulfil these requirements. We therefore suggest that further research should be undertaken on the practical implication of integrating donation management with menu planning. Besides considering the implementation of an MILP model, this could potentially be done by extending the heuristic decision rules of Section 5.6. For products with a short shelf life, decision rules may be well structured by the (recipe and ingredient) variety constraints. However, for products with a longer shelf life, decision rules are more complex and the integration of donation and menu planning decisions is even more relevant.

In this research several parameters are used which are stochastic in real life, such as the shelf life of a donation, or the chance that a donation will be available. Due to a scenario based approach and the rolling horizon, these stochastic parameters are integrated in the MILP model. For further research it can be interesting to develop a stochastic MILP to incorporate the stochastic nature of these parameters. Then, the objective function will be a minimization of the expected costs.

Even though we focused on soup kitchens in this chapter, more charitable organisations may benefit from similar approaches. Despite some differences between soup kitchens and food banks, our approach could likely be adjusted for a food bank setting in which food donations are used in the construction of food parcels. Deciding on food parcel contents would then replace the menu planning decisions. However, notable differences would likely be that food banks often do not purchase additional products (ingredients), and that transportation costs might be a more important consideration for food banks.

Besides soup kitchens, retailers benefit from donating leftovers by decreasing their food waste levels, and displaying only the freshest items. The greatest reduction in food waste can be obtained when good agreements are made between parties on donation quantities and costs are fairly shared. When costs are too high for either of the parties involved, leftovers will not be used optimally. In further research, we recommend to investigate how costs should be divided among the different parties. 

Chapter 6

Conclusions and general discussion 
The final chapter of this thesis will begin with a summary of the individual chapters, followed by an integrated discussion of the findings and the scientific contribution. Moreover, the implications of the interventions for different actors of the supply chain are explored and finally, suggestions for further research are proposed.

\subsection{Conclusions}

In the previous chapters, several interventions were analysed for their capacity to reduce food waste by retailers. Table 6.1 gives an overview of which interventions were studied in each chapter and which causes of food waste, as described in Chapter 1.2 and Figure 1.3, were tackled. Moreover, the table shows which performance indicator was used to analyse the effect of the intervention and whether the problem was solved by an optimisation or simulation-based optimisation model. As indicated in Table 6.1, store management was addressed in every chapter, as almost all the studied interventions require a change at the store-management level. Furthermore, the amount of profit or cost involved in each intervention was presented in each chapter. In the remainder of this section, a short summary of the main findings reported in each chapter is provided.

\subsubsection{Replenishment decisions with discounting and dynamic shelf life}

In Chapter 2, a reduction in food waste was obtained by optimising the replenishment quantity while implementing a dynamic shelf life and applying a discount to nearly expired products. The discount stimulated consumers who would otherwise prefer fresher products to buy the older, discounted products, controlling their in-store behaviour. The simulation study of a meat product showed around a $50 \%$ reduction in food waste when a $50 \%$ discount was applied by the retailer; however, profit levels decreased by an average of $5 \%$. In addition, a dynamic shelf life was implemented. The benefits of this were detected for multiple key performance indicators. In terms of waste, an average reduction of $41 \%$ was found when a dynamic shelf life was used. Moreover, the reduction of food waste led profit levels to increase (by up to 10\%) and the occurrence of product shortages was reduced. The main reason for these improvements was that the dynamic shelf life resulted in products having an extra day of shelf life, on average, in comparison with products with a fixed date. When there is more time to sell the product, retailers face less difficulty

in ensuring they have the right amount of each product in stock. The combined use of a dynamic shelf life and applying a discount resulted in even lower levels of absolute waste (around 1\%) than the independent application of either intervention. 
Table 6.1: Overview of focus in the different chapters of this thesis

\begin{tabular}{|c|c|c|c|c|c|}
\hline & & 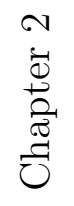 & 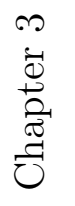 & 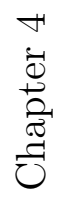 & 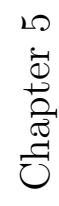 \\
\hline Cause & $\begin{array}{l}\text { Consumer behaviour and demand } \\
\text { Store management } \\
\text { Product shelf life }\end{array}$ & $\begin{array}{l}\checkmark \\
\checkmark \\
\checkmark\end{array}$ & $\begin{array}{l}\checkmark \\
\checkmark \\
\checkmark\end{array}$ & $\begin{array}{l}\checkmark \\
\checkmark \\
-\end{array}$ & $\begin{array}{l}- \\
\checkmark \\
\checkmark\end{array}$ \\
\hline Intervention & $\begin{array}{l}\text { Replenishment decisions } \\
\text { Assortment decisions } \\
\text { Discount } \\
\text { Dynamic shelf life } \\
\text { Substitution } \\
\text { Re-use of leftover food }\end{array}$ & $\begin{array}{l}\checkmark \\
- \\
\checkmark \\
\checkmark \\
- \\
-\end{array}$ & $\begin{array}{l}\checkmark \\
- \\
- \\
- \\
\checkmark \\
-\end{array}$ & $\begin{array}{l}\checkmark \\
\checkmark \\
- \\
- \\
\checkmark \\
-\end{array}$ & $\begin{array}{l}- \\
- \\
- \\
- \\
- \\
\checkmark\end{array}$ \\
\hline Performance indicator & $\begin{array}{l}\text { Food waste } \\
\text { Profit/costs } \\
\text { Service levels }\end{array}$ & $\begin{array}{l}\checkmark \\
\checkmark \\
\checkmark\end{array}$ & $\begin{array}{l}\checkmark \\
\checkmark \\
\checkmark\end{array}$ & - & $\begin{array}{l}\checkmark \\
\checkmark \\
-\end{array}$ \\
\hline Methodology & $\begin{array}{l}\text { Optimisation } \\
\text { Simulation }\end{array}$ & $\begin{array}{l}\checkmark \\
\checkmark\end{array}$ & $\begin{array}{l}\checkmark \\
\checkmark\end{array}$ & $\checkmark$ & $\checkmark$ \\
\hline
\end{tabular}




\subsubsection{Replenishment decisions with substitution-based ordering}

A reduction of waste was also obtained using a change in the replenishment strategy (Chapter 3). It is known that consumers are often willing to buy a substitute when their preferred product is out of stock (Gruen et al., 2002; Van Woensel et al., 2007); however, this is usually not considered in the replenishment decisions for (perishable) products. By combining the replenishment decisions for two perishable products in a one-way substitution model, the in-store consumer behaviour was incorporated into the replenishment decision. The reduction of waste for this two-product case was found to be $2-66 \%$, with an average decrease of $35 \%$. This reduction was highly dependent on the willingness of consumers to substitute products, the product shelf life and the differences in the profit margins of the two products. These findings revealed that, if many consumers were willing to buy a substitute if their preferred product was out of stock, it would be preferable from a retailer perspective to combine the demand for both products and only have one product in stock. This strategy resulted in high profit levels; however, it had a large effect on the service level.

\subsubsection{Replenishment and assortment decisions with substitution-based or- dering}

Chapter 4 also includes substitution behaviour of consumers in the replenishment decision. In the single period model presented in this chapter, assortment decisions were also incorporated, considering a multi-product problem. In this problem, multiple substitution rounds were incorporated, with all products being a possible substitution for each other. The results of this analysis showed that it is important to consider inventory levels when defining the optimal assortment to carry in the store. Moreover, the inclusion of multiple substitution rounds significantly affects the results when compared with models involving only a single substitution attempt, which is more commonly used in the literature. Although the presented model is not designed for products with a shelf life longer than one period, it still serves as a starting point for a multi-period problem. In line with the results of Chapter 3, profit levels increase when the substitution behaviour of the consumer is included.

\subsubsection{Re-use of food leftovers by a soup kitchen}

Aside from interventions aimed at reducing food waste at the retail level, food leftovers can also be utilised to reduce food waste. Chapter 5 shows the potential of the re-use of leftover food through donations to a soup kitchen. A MILP model, solved within a rolling horizon, was used to generate a meal plan for a soup kitchen. Depending on the 
size of the soup kitchen, the number of donations it receives, the constraints on the meals and the remaining shelf life of the donated products, $50-70 \%$ of the food waste from a retailer can be used. The main benefit of receiving food donations for a soup kitchen, or another charity organisation, is the cost reduction; donations mean less money must be spent to provide the meals. The shelf life of the donated products has a major influence on whether the donations can be used by the soup kitchen; for example, when constraints on menus and ingredient use are present (i.e., a limit on the number of times an ingredient or particular meal can be served during a seven-day period), donations with a very short shelf life cannot be used.

\subsection{Integrated findings and scientific contribution}

All interventions studied in this thesis showed a potential for reducing food waste at the retail level, and therefore contribute to the overall research aim, as formulated in Chapter 1.4:

Overall research aim: To analyse the impact of several interventions for reducing food waste at the retail level from an Operations Research perspective.

In addition to reducing waste, the majority of these interventions also maintain or even increase the profit levels of the retailer. When combined, the interventions can have a synergistic effect that surpass those of the individual approaches. These integrated findings are now discussed.

\subsubsection{Holistic view on food waste}

Food waste reduction at the retailer cannot be seen as a stand-alone action due to the interdependencies at the retail level itself. Interactions between the retailer and other supply chain actors are discussed in section 6.3. Both interdependencies between the different products as dependencies between the different performance measures of the retailer. First, if a reduction in food waste is obtained via a price discount, price-based substitution will occur; the discounted product convinces consumers to purchase the "older" product instead of a fresh one, but it can also convince other consumers who intended to buy a different product to instead substitute towards the discounted product. The demand of other products can therefore decrease, resulting in a cannibalisation of demand. These products are most likely perishable as well, and this reduced demand leads to increased waste levels for these products. Second, food waste reduction goes hand in hand with 
the performance indicators of profit and service level. Profit levels can increase when the products at the retailer are sold rather than wasted; however, this can reduce the service level. If service levels are to be maintained while reducing the waste levels, profit levels cannot be maintained. All these interdependencies demonstrate the need for a holistic view on food waste to achieve a sustainable reduction.

\subsubsection{Anticipation of food waste}

Food waste at the retail level can be reduced; however, it is impossible to avoid waste completely. It is therefore very important to explore options to further re-use food leftovers, for example by donating them to charity organisations such as a soup kitchen or food bank. This re-use will reduce the environmental impact of our food system, and has great social value. The remaining shelf life of the leftovers plays an important role in their

efficient re-use. Not all retailers sell products until the day of the use-by or best-before date. Some supermarkets only want to sell very fresh products to their customers, and thus discard products with two days of shelf life remaining. For on-line grocery shopping, products delivered to customers usually have several days of shelf life left. In these situations, the discarded products are particularly suitable for donation to charity; however, the retailer should anticipate this (re-occurring) flow of discarded products upfront, enabling a good logistics system to be established to transfer food leftovers from the retailer to a secondary outlet, such as a soup kitchen or food bank.

\subsubsection{Methodological contribution}

In this thesis, multiple Operations Research techniques are used. The simulation-based optimisation approach allows the exploration and solving of problems representing realistic situations for a retailer. Simulation models allow a good representation of the variability in the system, and provide an accurate view of its effects (Robinson, 2004). Simulation models therefore allow the implementation of stochastic demand, a limited product shelf life and lead times. The inclusion of these settings increases the reality of the represented environment in which a retailer operates; however, they increase the complexity of the model. The drawback of highly complex models is twofold: it increases the computational time needed to solve the model and reduces its tractability. Moreover, it is not always necessary to include as many details as possible in a model to get the best result. Depending on which problem is solved, assumptions can be made to limit the model complexity without affecting the outcomes.

In this study, both exact and heuristic solution methods were used to solve the (simulationbased) optimisation models. Exact methods will give the optimal solution; however, when 
the model complexity increases, these methods cannot always be used, either because the computational time needed becomes too large or because the problem is not tractable any more. In these cases, heuristics can be used, which do not guarantee the optimal solution; however, if implemented well, good solutions can be found. An example of the need for and use of heuristics is found in this thesis. The model considering the multiproduct problem for the single period can be solved using exact solution methods within a reasonable time for four products; however, when more products would be included, this approach is no longer reasonable. The heuristic approach used solved the model to (near-) optimal solutions, and thus should be used for the larger problems. Besides simulationbased optimisation modelling, this thesis uses MILP, a pure optimisation technique. A major benefit of (MI)LP models is their applicability for solving many problems related to the supply chain. They can be used to find the best delivery route, optimal network configurations (e.g., where to place a hub) or how many acres of land should be used for a certain crop (de Keizer et al., 2014; Jonkman et al., 2019a; Rohmer et al., 2019). Finding an optimal meal plan is a typical optimisation problem with linear relationships; therefore, a linear programming approach was used. A drawback of MILP models is their exclusion of uncertainty. To consider the uncertainty of donation information in the menu-planning problem, a rolling horizon algorithm was used.

\subsubsection{Contribution to the literature}

This thesis makes several contributes to the existing literature. The technical aspects of a dynamic shelf life, such as sensors to measure the quality of a food product, have been studied previously (Heising, 2014; Kreyenschmidt et al., 2010); however, the consequences for the retailer in terms of food waste, replenishment quantities, profit or service levels have not. The effect of discounts on replenishment quantities had not been studied under stochastic demand. Chapter 1.3 discusses the lack of scientific research related to multiitem inventory management, while Chapter 3 considers an inventory problem involving two products with short shelf lives and a stock-out based substitution. Previous studies investigating a multi-item replenishment problem under consumer-driven substitution have either dealt with a single-period problem or did not include product perishability.

Chapter 4 makes several contributions to the literature. The results reported in this chapter show that the inclusion of multiple substitution rounds outperforms the common use of only one round of substitutions, while also demonstrating the importance of including inventory levels to determine the optimal assortment of a product category. This chapter also provides a framework to determine the changing substitution fractions for every substitution round, and moreover, a heuristic is developed to find (near-) optimal solutions for this complex problem. Previous studies have shown the amount of food that can be 
recovered from the supermarket, but there is limited research on the re-use of this recovered food (Alexander \& Smaje, 2008; Cicatiello et al., 2016). The menu-planning model provides a framework for the optimisation of food re-use from donations provided by supermarkets. In the literature, many interventions to reduce food waste are mentioned; however, their effects are rarely quantified (Teller et al., 2018). This thesis provides the quantification for some of the possible interventions.

\subsection{Managerial discussion}

The interventions studied in this thesis do not influence the retailer alone. The retailer is only one of the actors in the food supply chain, and therefore, interventions implemented by retailers also affect the other supply chain actors. This section discusses the implications for the retailer, upstream and downstream actors, as well as the implications for society in general.

\subsubsection{Implications for the retailer}

Besides profit and waste levels, service levels are an important performance measure for the retailer. Retailers want to provide the best service as possible for their customers, and thus strive towards high service levels; however, very high service levels often result in very high levels of waste (Haijema \& Minner, 2019). Furthermore, food waste can have a negative effect on service levels. When the replenishment decision is based on the current inventory without anticipating for possible waste, a shortage might occur the next day, as there are fewer products in stock than expected; however, if the replenishment strategy accounts for possible food waste, the number of shortages decrease (i.e., the service levels increase).

The interventions analysed in this thesis do not always increase the service level for each product. In Chapter 3, the overall service level of the product group increases, but the service level of each individual product decreases with substitution-based ordering. In Chapter 4, service levels are not considered; however, when the results obtained with the combined ordering strategy for multiple products is compared with the newsvendor model, a significant change is visible. When the substitution behaviour of consumers is included, the optimal stock quantities increase. Higher stock levels contribute to higher (overall) service levels; however, this does not have to be true for each product. Moreover, when replenishment decisions for products are combined, the retailer must define which products belong to the same product category and can thus serve as a substitute. Consumer research can be used to help the retailer define these product categories. 


\subsubsection{Implications for the upstream supply chain actors}

The dynamic shelf life intervention has a direct effect on the upstream supply chain. In order to apply this intervention, the upstream actors of the supply chain, especially the food manufacturers, will necessarily be involved. Currently, the food manufacturer decides, based on shelf life experiments, what the shelf life of the product will be, and thus which date is printed on the product (Soethoudt et al., 2012). In implement a dynamic shelf life, the manufacturer has to apply something like a sensor or RFID tag rather than a fixed date.

The other studied interventions have less of a direct impact on the upstream actors of the supply chain. A change in ordering strategy would likely affect the retailer more than the upstream actors; however, it may still impact the food producers and suppliers. A wellknown example is the bullwhip effect, which occurs due to an uncertainty in consumer demand and the response of the retailer to this uncertainty. To cope with the variation in demand, a safety stock is held by the retailer, meaning that the amount of product ordered from their supplier might not align with actual consumer demands (Forrester, 1961). Although a short shelf life reduces the bullwhip effect (Minner \& Transchel, 2017), a change in ordering strategy can still have an undesired effect. Donating products to a charity organisation before they pass their shelf life can also increase the orders placed at the distribution centre or manufacturer. Many studies show that information sharing or vendor-managed inventory (VMI) can improve the performance of the entire supply chain in response to order variability (Wang \& Disney, 2016). Such solutions can be considered when improvements are attempted from a broader supply chain perspective, or if the interventions implemented at the retail level have a negative effect on the performance of the upstream actors.

\subsubsection{Implications of interventions for the consumer}

Food waste at the household level is high; around $40 \%$ of the food waste occurs in the homes of consumers (Gustavsson et al., 2011). Household food waste occurs for various reasons; however, one of the main reasons is the shopping behaviour of consumers (Roodhuyzen et al., 2017). Retailer strategies can therefore directly influence food waste at the household level (Mondéjar-Jiménez et al., 2016). Discounts or bulk promotions are a trigger for consumers to purchase more products, often more than they can eat before the expiration date (Farr-Wharton et al., 2014; Graham-Rowe et al., 2014; Roodhuyzen et al., 2017). To achieve a sustainable reduction in food waste, the retail sector should be aware of their influence on consumer food waste. When interventions at the retailer reduce food waste at the supermarket but lead to more food waste at the household level, 
further improvements are needed.

Another cause of food waste at home is related to the perishability of products. Consumers do not always consume the products before the shelf life has passed (Aschemann-Witzel et al., 2017). If products sold at the supermarket had a longer remaining shelf life (i.e., the products were fresher), consumers have more time to use the product and therefore household waste might decrease. Chapter 2 shows that products with a dynamic shelf life have an increased freshness at the moment of consumer purchase. Furthermore, Ferguson \& Ketzenberg (2005) previously showed that information sharing between the supply chain actors leads to fresher products at the supermarket. When interventions result in a better alignment of supply and demand at the retailer, the average time products spend on the shelf is reduced, giving the consumer more time to use the products at home (Broekmeulen \& van Donselaar, 2019).

\subsubsection{Implications for society in general}

Reducing food waste at any stage of the food supply chain affects the entire system. Most food is produced in countries with warmer climates, such as those in tropical or subtropical regions, then transported to every country in the world (Khoury et al., 2016). The food waste that occurs in Europe therefore has an effect not only on the food system within the EU, but also on the global food system. A reduction of EU food waste would thus reduce the need to import food from other places, decreasing transportation costs and emissions and reducing the contribution of European food consumption to the global warming potential (GWP). Moreover, a more equal distribution of food among the world's population could be established. Currently, around $11 \%$ of the global population faces food insecurity (FAO, 2018). A reduction in the European waste of food sourced from around the world should result in greater food availability in other parts of the world.

\subsubsection{Application of models and interventions to other supply chains and other supply chain actors}

The focus of this thesis is on the food supply chain; however, this is not the only supply chain that deals with waste. In terms of quality decay and perishability, the horticultural sector is very similar to the food sector (de Keizer et al., 2017); therefore, the interventions studied in this thesis and the developed modelling approaches will also be of value to this sector. Furthermore, the electronic and fashion sectors have many similarities to the food supply chains. Fashion trends change rapidly, resulting in short product life cycles; moreover, demand is unpredictable and a huge variety of products exist (Sen, 2008). Within the supply chains of electronic products, product substitution can occur to a 
large extent (Chen et al., 2015); for example, when a new version of a smartphone is introduced, the previous version will become almost obsolete, although some consumers will still decide to buy the older, and usually cheaper, version. Some of the interventions studied in this thesis, such as discounting or the inclusion of substitution behaviours, can be applied to fashion or electronic products. This extends the applicability of the models and interventions of this thesis to a larger range of products.

\subsection{Further research}

Inspired by the results of this thesis, several ideas for further research are discussed in this section, including suggestions to extend the current research, ways to overcome some of the limitations in this study, and new research ideas to reduce food waste at the retail level. The results described in this thesis also inspire further research on a broader range of topics related to retail analytics. This thesis includes some of the interventions that reduce food waste at the retailer; however, the possibilities are not limited to the ones proposed here.

Consumer in-store behaviour, such as the response to product quality, price and assortment, is incorporated into most of the models developed in this thesis; however, our understanding of how consumers actually behave is limited, and assumptions have to be made to obtain results. Some studies have investigated consumer responses to stock-outs (e.g. Gruen et al., 2002; Van Woensel et al., 2007); furthermore, factors influencing demand for a product or factors that initiate or stimulate product substitution have been (extensively) studied from a marketing point of view (e.g. Jacobson \& Aaker, 1987; Boatwright $\&$ Nunes, 2001). The outcomes of these studies can be used for a good approximation of the assumption on consumer behaviour; however, the results of these studies might differ between various product or consumer groups. The assumptions made regarding consumer responses to discounts on nearly expired products are also worth further study. Consumers are price sensitive in general; however, their willingness to pay reduces when products are of lower quality or have a shorter remaining shelf life (Han et al., 2001; Tsiros \& Heilman, 2005). More research on in-store consumer behaviour is therefore needed, not only in a qualitative manner (i.e., reasons why consumers behave as they do), but also with a quantitative approach to define the size of the effects. This would contribute towards the generation of more robust results. In terms of the provision of a discount on nearly expired products, it would be interesting to consider the whole product category rather than one specific product. Consumers are price sensitive, and thus price-based substitution might occur when a product is discounted at the end of its shelf life $(\mathrm{Hu}$ et al., 2016). This substitution within the product (i.e., LEFO to FEFO) is incorporated 
in the model in Chapter 2; however, consumers who initially prefer another product might also decide to go for the discounted product. When this happens, the discount affects both the inventory and waste levels of the discounted product and other products in the category.

In addition to making replenishment decisions based on all products within a product category, other factors can be taken into account to make replenishment even more efficient. The age or remaining shelf life of the products could be included; for example, the retailer might decide not to order one product when many items of another product in the same category are still available, in order to first sell the older products.

Within the literature on inventory management or substitution, previous studies have considered either multi-product problems or multi-period problems, and use solving approaches for larger problems (Shin et al., 2015; Janssen et al., 2016). In future research, it will be of great interest to study multi-product assortment and inventory problems in a multi-period setting. This will enhance the applicability of the models to real-life practice because many (highly) perishable products are sold by retailers. This extension will require advanced solving methods because the problem complexity will increase. One possibility is to use the heuristic approach developed in Chapter 4 and include it within a simulation environment. Another possibility would be to analyse existing solving methods for their ability to cope with the problem complexity.

In Chapter 1, the environmental impact of the food system and especially food waste was addressed; however, for the interventions studied in this thesis, only the economic value of food waste is considered, which might not align with the environmental impact (Scholz et al., 2015). Previous research has shown that trade-offs can occur between economic and environmental objectives (Jonkman et al., 2019b). By focussing on the environmental impact as well as the economical side of food waste, the results may be affected. In future studies, it will be useful to study not only the trade-off between profit and service levels, but include environmental indicators as well. As discussed in Chapter 6.3, the retailer has a significant influence on food waste at the household level; however, the exact link between the decisions made at the retail level and the food waste levels of households are not yet fully understood. To address the food waste problem throughout the entire food supply chain, it is important to study these linkages between actors in the supply chain. Retailers have a particularly large influence on the other actors of the food supply chain; therefore, further investigations should be made into the interactions between the decisions made by a retailer and the consequences for the consumer. Large pack sizes are one of the causes of food waste at the household level (Aschemann-Witzel et al., 2017), and also cause challenges for retailers when products can only be ordered in fixed batches (Broekmeulen \& van Donselaar, 2019). When the batch sizes are larger than the average demand for the product within the shelf life, product will be wasted. Studying batch 


\section{Conclusions and general discussion}

sizes can be combined with the research on product substitution presented in this thesis. When a retailer cannot order the desired amount of items, product substitution by the consumer should be taken into account in an attempt to reduce food waste. Instead of rounding every order up to the nearest available batch size, ordering decisions for various products can be combined, such that some orders can be rounded down while others are rounded up. The resulting limited availability of some products will force some consumers to buy a substitute product, but this approach could improve the overall performance of the retailer.

There is a strong link between food waste and service levels (Haijema, 2014); however, service levels are not the only factor influencing the amount of food waste at the supermarket. It is therefore interesting to study whether the level of food waste can be predicted based on a pre-defined service level and other factors. Sales data from the supermarket might also provide a lot of insight when thoroughly analysed. The prediction of food waste using multiple factors will likely improve the performance of the retailer.

Food waste will remain a very important topic to address in the future, and the list of research topics is not limited to the suggestions given in this chapter. Like most retail markets, grocery retail is experiencing an ongoing shift towards omni-channel retailing (Wollenburg et al., 2018), which requires and allows for more research into food waste and retail management in general. 



\section{References}

Akçay, Y., Natarajan, H. P., \& Xu, S. H. (2010). Joint dynamic pricing of multiple perishable products under consumer choice. Management Science, 56. doi: 10.1287/ mnsc. 1100.1178 .

Alexander, C., \& Smaje, C. (2008). Surplus retail food redistribution: An analysis of a third sector model. Resources, Conservation and Recycling, 52. doi: 10.1016/ j.resconrec.2008.07.009.

Aschemann-Witzel, J., Jensen, J. H., Jensen, M. H., \& Kulikovskaja, V. (2017). Consumer behaviour towards price-reduced suboptimal foods in the supermarket and the relation to food waste in households. Appetite, 116. doi: 10.1016/j.appet.2017.05.013.

Avinadav, T., Herbon, A., \& Spiegel, U. (2013). Optimal inventory policy for a perishable item with demand function sensitive to price and time. International Journal of Production Economics, 144. doi: 10.1016/j.ijpe.2013.03.022.

Bakker, M., Riezebos, J., \& Teunter, R. H. (2012). Review of inventory systems with deterioration since 2001. European Journal of Operational Research, 221 . doi: 10.1016/ j.ejor.2012.03.004.

Balcik, B., Iravani, S., \& Smilowitz, K. (2014). Multi-vehicle sequential resource allocation for a nonprofit distribution system. IIE Transactions, 46. doi: 10.1080/ 0740817X.2013.876240.

Balintfy, J. L. (1964). Menu planning by computer. Communications of the ACM, 7 . doi: $10.1145 / 364005.364087$.

Bas, E. (2014). A robust optimization approach to diet problem with overall glycemic load as objective function. Applied Mathematical Modelling, 38. doi: 10.1016/ j.apm.2014.03.049.

Beretta, C., Stoessel, F., Baier, U., \& Hellweg, S. (2013). Quantifying food losses and the potential for reduction in Switzerland. Waste Management, 33. doi: 10.1016/ j.wasman.2012.11.007.

Berk, E., Gürler, Ü., \& Yıldırım, G. (2009). On pricing of perishable assets with 
menu costs. International Journal of Production Economics, 121. doi: 10.1016/ j.ijpe.2009.02.010.

Berry, S., \& Pakes, A. (2007). The pure characteristics demand model. International Economic Review, 48. doi: 10.1111/j.1468-2354.2007.00459.x.

Boatwright, P., \& Nunes, J. C. (2001). Reducing assortment: an attribute-based approach. Journal of Marketing, 65. doi: 10.1509/jmkg.65.3.50.18330.

Brock, L. G., \& Davis, L. B. (2015). Estimating available supermarket commodities for food bank collection in the absence of information. Expert Systems with Applications, 42. doi: 10.1016/j.eswa.2014.11.068.

Broekmeulen, R. A., \& van Donselaar, K. H. (2009). A heuristic to manage perishable inventory with batch ordering, positive lead-times, and time-varying demand. Computers E Operations Research, 36. doi: 10.1016/j.cor.2009.01.017.

Broekmeulen, R. A., \& van Donselaar, K. H. (2019). Quantifying the potential to improve on food waste, freshness and sales for perishables in supermarkets. International Journal of Production Economics, 209. doi: 10.1016/j.ijpe.2017.10.003.

Bruckner, S. (2010). Predictive shelf life model for the improvement of quality management in meat chains. Ph.D. thesis, Universitäts-und Landesbibliothek Bonn, Germany.

Buisman, M., Haijema, R., \& Bloemhof-Ruwaard, J. (2019a). Discounting and dynamic shelf life to reduce fresh food waste at retailers. International Journal of Production Economics, 209. doi: 10.1016/j.ijpe.2017.07.016.

Buisman, M. E., Haijema, R., Akkerman, R., \& Bloemhof, J. M. (2019b). Donation management for menu planning at soup kitchens. European Journal of Operational Research, 272. doi: 10.1016/j.ejor.2018.06.005.

Buzby, J. C., Hyman, J., Stewart, H., \& Wells, H. F. (2011). The value of retail- and consumer-level fruit and vegetable losses in the United States. Journal of Consumer Affairs, 45. doi: 10.1111/j.1745-6606.2011.01214.x.

Cachon, G. P., Terwiesch, C., \& Xu, Y. (2005). Retail assortment planning in the presence of consumer search. Manufacturing \& Service Operations Management, \%. doi: 10.1287/ msom.1050.0088.

Cadenas, J. M., Pelta, D. A., Pelta, H. R., \& Verdegay, J. L. (2004). Application of fuzzy optimization to diet problems in Argentinean farms. European Journal of Operational Research, 158. doi: 10.1016/S0377-2217(03)00356-4.

CalRecycle (2017). Mandatory Commercial Organics Recycling. Retrieved from http: //www.calrecycle.ca.gov/recycle/commercial/organics/.

CBS (2018). StatLine - Laag en langdurig laag inkomen van huishoudens; huishoudensken- 
merken. Retrieved from https://opendata.cbs.nl/statline/\{\\#\}/CBS/nl/dataset/ 83841NED/table?ts=1550743485515.

Chand, S., Ward, J. E., \& Kevin Weng, Z. (1994). A parts selection model with oneway substitution. European Journal of Operational Research, 73. doi: 10.1016/03772217(94)90143-0.

Chen, X., Feng, Y., Keblis, M. F., \& Xu, J. (2015). Optimal inventory policy for two substitutable products with customer service objectives. European Journal of Operational Research, 246. doi: 10.1016/j.ejor.2015.04.033.

Chew, E. P., Lee, C., Liu, R., Hong, K. S., \& Zhang, A. (2014). Optimal dynamic pricing and ordering decisions for perishable products. International Journal of Production Economics, 157. doi: 10.1016/j.ijpe.2013.12.022.

Chrisafis, A. (2016). French law forbids food waste by supermarkets. Retrieved from https : //www.theguardian.com/world/2016/feb/04/french-law-forbids-foodwaste-by-supermarkets.

Chung, K.-J., \& Lin, C.-N. (2001). Optimal inventory replenishment models for deteriorating items taking account of time discounting. Computers $\&$ Operations Research, 28. doi: 10.1016/S0305-0548(99)00087-8.

Cicatiello, C., Franco, S., Pancino, B., \& Blasi, E. (2016). The value of food waste: An exploratory study on retailing. Journal of Retailing and Consumer Services, 30. doi: 10.1016/j.jretconser.2016.01.004.

Cicatiello, C., Franco, S., Pancino, B., Blasi, E., \& Falasconi, L. (2017). The dark side of retail food waste: Evidences from in-store data. Resources, Conservation and Recycling, 125. doi: 10.1016/j.resconrec.2017.06.010.

Davis, L. B., Sengul, I., Ivy, J. S., Brock, L. G., \& Miles, L. (2014). Scheduling food bank collections and deliveries to ensure food safety and improve access. Socio-Economic Planning Sciences, 48. doi: 10.1016/j.seps.2014.04.001.

Deniz, B., Karaesmen, I., \& Scheller-Wolf, A. (2010). Managing perishables with substitution: Inventory issuance and replenishment heuristics. Manufacturing \& Service Operations Management, 12. doi: 10.1287/msom.1090.0276.

Dillon, M., Oliveira, F., \& Abbasi, B. (2017). A two-stage stochastic programming model for inventory management in the blood supply chain. International Journal of Production Economics, 187. doi: 10.1016/j.ijpe.2017.02.006.

van Donselaar, K., van Woensel, T., Broekmeulen, R., \& Fransoo, J. (2006). Inventory control of perishables in supermarkets. International Journal of Production Economics, 104. doi: 10.1016/j.ijpe.2004.10.019. 
Drezner, Z., Gurnani, H., \& Pasternack, B. A. (1995). An EOQ model with substitutions between products. Journal of the Operational Research Society, 46. doi: 10.1057/ jors.1995.120.

Duan, Q., \& Liao, T. W. (2014). Optimization of blood supply chain with shortened shelf lives and ABO compatibility. International Journal of Production Economics, 153. doi: 10.1016/j.ijpe.2014.02.012.

Elmaghraby, W., \& Keskinocak, P. (2003). Dynamic pricing in the presence of inventory considerations : Research overview, current practices and future directions. Management Science, 49. doi: 10.1287/mnsc.49.10.1287.17315.

Eppich, S., \& Fernandez, C. P. (2004). Study finds Chapel Hill, NC, soup kitchen serves nutritious meals. Journal of the American Dietetic Association, 104. doi: 10.1016/ j.jada.2004.05.208.

Eriksson, M., Strid, I., \& Hansson, P.-A. (2014). Waste of organic and conventional meat and dairy products - A case study from Swedish retail. Resources, Conservation and Recycling, 83. doi: 10.1016/j.resconrec.2013.11.011.

Ernst, R., \& Kouvelis, P. (1999). The effects of selling packaged goods on inventory decisions. Management Science, 45. doi: 10.1287/mnsc.45.8.1142.

FAO (2018). SOFI 2018 - The state of food security and nutrition in the world. Retrieved from http://www.fao.org/state-of-food-security-nutrition/en/.

Farr-Wharton, G., Foth, M., \& Choi, J. H. J. (2014). Identifying factors that promote consumer behaviours causing expired domestic food waste. Journal of Consumer Behaviour, 13. doi: 10.1002/cb.1488.

Farughi, H., Khanlarzade, N., \& Yegane, B. (2014). Pricing and inventory control policy for non-instantaneous deteriorating items with time-and price-dependent demand and partial backlogging. Decision Science Letters, 3.

Ferguson, M. E., \& Ketzenberg, M. E. (2005). Information sharing to improve retail product freshness of perishables. Production and Operations Management, 15.

Forrester, J. W. (1961). Industrial dynamics. Ph.D. thesis, Cambridge, MA, United States.

Gao, H., Wang, D., Santibanez Gonzalez, E. D. R., \& Ju, Y. (2019). Optimal stocking strategies for inventory mechanism with a stochastic short-term price discount and partial backordering. International Journal of Production Research, . doi: 10.1080/ 00207543.2019.1567949.

Gaur, V., \& Honhon, D. (2006). Assortment planning and inventory decisions under a locational choice model. Management Science, 52. doi: 10.1287/mnsc.1060.0580. 
Ghoniem, A., Scherrer, C. R., \& Solak, S. (2012). A specialized column generation approach for a vehicle routing problem with demand allocation. Journal of the Operational Research Society, 64. doi: 10.1057/jors.2012.32.

Godfray, H. C. J., Beddington, J. R., Crute, I. R., Haddad, L., Lawrence, D., Muir, J. F., Pretty, J., Robinson, S., Thomas, S. M., \& Toulmin, C. (2010). Food security: the challenge of feeding 9 billion people. Science, 327. doi: 10.1126/science.1185383.

Goyal, S., \& Giri, B. (2001). Recent trends in modeling of deteriorating inventory. European Journal of Operational Research, 134. doi: 10.1016/S0377-2217(00)00248-4.

Graham-Rowe, E., Jessop, D. C., \& Sparks, P. (2014). Identifying motivations and barriers to minimising household food waste. Resources, Conservation and Recycling, 84. doi: 10.1016/j.resconrec.2013.12.005.

Gruber, V., Holweg, C., \& Teller, C. (2016). What a waste! Exploring the human reality of food waste from the store manager's perspective. Journal of Public Policy 8 Marketing, 35. doi: 10.1509/jppm.14.095.

Gruen, T. W., Corsten, D. S., \& Bharadwaj, S. (2002). Retail out-of-stocks: a worldwide examination of extent, causes and consumer responses. Grocery Manufacturers of America, Washington DC, United States. Retrieved from http://itsoutof stock.com/ wp-content/uploads/2013/04/GMA $\left\{\backslash_{-}\right\} 2002\left\{\backslash_{-}\right\}$-Worldwide $\left\{\backslash_{-}\right\} 00 S\left\{\backslash_{-}\right\}$Study.pdf.

Guley, H. M., \& Stinson, J. P. (1984). Scheduling and resource allocation in a food service system. Journal of Operations Management, 4. doi: 10.1016/0272-6963(84)90028-7.

Gustavsson, J., Cederberg, C., \& Sonesson, U. (2011). Global food losses and food waste. Rome: Food and Agriculture Organization of the United Nations. FAO, Rome, Italy. Retrieved from http://www.fao.org/3/a-i2697e.pdf.

Gustavsson, J., \& Stage, J. (2011). Retail waste of horticultural products in Sweden. Resources, Conservation and Recycling, 55. doi: 10.1016/j.resconrec.2011.01.007.

Haijema, R. (2014). Optimal ordering, issuance and disposal policies for inventory management of perishable products. International Journal of Production Economics, 157. doi: 10.1016/j.ijpe.2014.06.014.

Haijema, R., \& Minner, S. (2016). Stock-level dependent ordering of perishables: A comparison of hybrid base-stock and constant order policies. International Journal of Production Economics, 181. doi: 10.1016/j.ijpe.2015.10.013.

Haijema, R., \& Minner, S. (2019). Improved ordering of perishables: The value of stockage information. International Journal of Production Economics, 209. doi: 10.1016/ j.ijpe.2018.03.008.

Haijema, R., van der Wal, J., \& van Dijk, N. M. (2005). Blood Platelet Production: a 
multi-type perishable inventory problem. (pp. 84-92). Springer, Berlin, Heidelberg. doi: 10.1007/3-540-27679-3_11.

Han, G., Dong, M., \& Shao, X. (2012). Yield management with downward substitution and uncertainty demand in semiconductor manufacturing. International Journal of Production Research, 50. doi: 10.1080/00207543.2010.543942.

Han, S., Gupta, S., \& Lehmann, D. R. (2001). Consumer price sensitivity and price thresholds. Journal of Retailing, 77. doi: 10.1016/S0022-4359(01)00057-4.

Harris, B. (2017). Courtauld 2025 signatory data report: 2015 and 2016. WRAP, Banbury, United Kingdom. Retrieved from http://www.wrap.org.uk/sites/files/wrap/ CourtauldCommitment2025-baselinereportfor2015.pdf.

Heising, J. K. (2014). Intelligent packaging for monitoring food quality: a case study on fresh fish. Ph.D. thesis, Wageningen University, Wageningen, the Netherlands.

Hendrix, E. M. T., Ortega, G., Haijema, R., Buisman, M. E., \& García, I. (2019). On computing optimal policies in perishable inventory control using value iteration. Computational and Mathematical Methods, 1. doi: 10.1002/cmm4.1027.

Herbon, A., Levner, E., \& Cheng, E. (2012). Perishable inventory management and dynamic pricing using TTI technologies. International Journal of Innovation, Management and Technology, 30 .

Honhon, D., Gaur, V., \& Seshadri, S. (2010). Assortment planning and inventory decisions under stockout-based substitution. Operations Research, 58. doi: 10.1287/ opre.1090.0805.

Honhon, D., \& Seshadri, S. (2013). Fixed vs. random proportions demand models for the assortment planning problem under stockout-based substitution. Manufacturing $\&$ Service Operations Management, 15. doi: 10.1287/msom.1120.0425.

Hopp, W. J., \& Xu, X. (2005). Product line selection and pricing with modularity in design. Manufacturing \& Service Operations Management, 7. doi: 10.1287/ msom.1050.0077.

Hu, P., Shum, S., \& Yu, M. (2016). Joint inventory and markdown management for perishable goods with strategic consumer behavior. Operations Research, 64. doi: 10.1287/opre.2015.1439.

Hübner, A., Kuhn, H., \& Kühn, S. (2016). An efficient algorithm for capacitated assortment planning with stochastic demand and substitution. European Journal of Operational Research, 250. doi: 10.1016/j.ejor.2015.11.007.

Hübner, A., \& Schaal, K. (2017). An integrated assortment and shelf-space optimization model with demand substitution and space-elasticity effects. European Journal of 
Operational Research, 261. doi: 10.1016/j.ejor.2017.01.039.

Hübner, A. H., \& Kuhn, H. (2012). Retail category management: State-of-the-art review of quantitative research and software applications in assortment and shelf space management. Omega, 40. doi: 10.1016/j.omega.2011.05.008.

Jacobson, R., \& Aaker, D. A. (1987). The strategic role of product quality. Journal of Marketing, 51. doi: 10.2307/1251246.

Janssen, L., Claus, T., \& Sauer, J. (2016). Literature review of deteriorating inventory models by key topics from 2012 to 2015. International Journal of Production Economics, 182. doi: 10.1016/j.ijpe.2016.08.019.

Jonkman, J., Barbosa-Póvoa, A. P., \& Bloemhof, J. M. (2019a). Integrating harvesting decisions in the design of agro-food supply chains. European Journal of Operational Research, 276. doi: 10.1016/j.ejor.2018.12.024.

Jonkman, J., Kanellopoulos, A., \& Bloemhof, J. M. (2019b). Designing an eco-efficient biomass-based supply chain using a multi-actor optimisation model. Journal of Cleaner Production, 210. doi: 10.1016/j.jclepro.2018.10.351.

Karaesmen, I. Z., Scheller-Wolf, A., \& Deniz, B. (2011). Managing perishable and aging inventories: Review and future research directions. (pp. 393-436). Springer US. doi: 10.1007/978-1-4419-6485-4_15.

de Keizer, M., Akkerman, R., Grunow, M., Bloemhof, J. M., Haijema, R., \& van der Vorst, J. G. (2017). Logistics network design for perishable products with heterogeneous quality decay. European Journal of Operational Research, 262. doi: 10.1016/j.ejor.2017.03.049.

de Keizer, M., Groot, J. J., Bloemhof, J., \& van der Vorst, J. G. (2014). Logistics orchestration scenarios in a potted plant supply chain network. International Journal of Logistics Research and Applications, 17. doi: 10.1080/13675567.2013.837157.

Ketzenberg, M., Bloemhof, J., \& Gaukler, G. (2015). Managing perishables with time and temperature history. Production and Operations Management, 24. doi: 10.1111/ poms.12209.

Khouja, M., Mehrez, A., \& Rabinowitz, G. (1996). A two-item newsboy problem with substitutability. International Journal of Production Economics, 44. doi: 10.1016/ 0925-5273(96)80002-V.

Khoury, C. K. et al. (2016). Origins of food crops connect countries worldwide. Proceedings of the Royal Society B: Biological Sciences, 283. doi: 10.1098/rspb.2016.0792.

Kok, A. G. (2003). Management of product variety in retail operations. Ph.D. thesis, University of Pennsylvenia, United States. 
Kök, A. G., \& Fisher, M. L. (2007). Demand estimation and assortment optimization under substitution: Methodology and application. Operations Research, 55. doi: 10.1287/opre.1070.0409.

Kök, G. A., Fisher, M. L., \& Vaidyanathan, R. (2008). Assortment planning: Review of literature and industry practice. (pp. 99-153). Springer US. doi: 10.1007/978-0-38778902-6_6.

Kreyenschmidt, J., Christiansen, H., Hübner, A., Raab, V., \& Petersen, B. (2010). A novel photochromic time-temperature indicator to support cold chain management. International Journal of Food Science \& Technology, 45. doi: 10.1111/j.13652621.2009.02123.x.

Lancaster, L. M. (1992). The history of the application of mathematical programming to menu planning. European Journal of Operational Research, 57. doi: 10.1016/03772217(92)90345-A.

Lebersorger, S., \& Schneider, F. (2014). Food loss rates at the food retail, influencing factors and reasons as a basis for waste prevention measures. Waste Management, 34. doi: 10.1016/j.wasman.2014.06.013.

Lee, D., \& Tongarlak, M. H. (2017). Converting retail food waste into by-product. European Journal of Operational Research, 257. doi: 10.1016/j.ejor.2016.08.022.

Leung, P., Wanitprapha, K., \& Quinn, L. a. (1995). A recipe-based, diet-planning modelling system. The British journal of nutrition, 74. doi: 10.1079/bjn19950119.

Li, Z. (2009). A single-period assortment optimization model. Production and Operations Management, 16. doi: 10.1111/j.1937-5956.2007.tb00265.x.

Lien, R. W., Iravani, S. M. R., \& Smilowitz, K. R. (2014). Sequential resource allocation for nonprofit operations. Operations Research, 62. doi: 10.1287/opre.2013.1244.

Lin, F., Yang, Z.-C., \& Jia, T. (2016). Optimal pricing and ordering policies for non instantaneous deteriorating items with price dependent demand and maximum lifetime. In Proceedings of the 6th International Asia Conference on Industrial Engineering and Management Innovation (pp. 411-421). Springer.

Liu, X., Tang, O., \& Huang, P. (2008). Dynamic pricing and ordering decision for the perishable food of the supermarket using RFID technology. Asia Pacific Journal of Marketing and Logistics, 20. doi: 10.1108/13555850810844841.

Lundqvist, J., de Fraiture, C., \& Molden, D. (2008). Saving water: From field to fork - Curbing losses and wastage in the food chain. SIWI Policy Brief, Stockholm, Sweden. Retrieved from https://www.siwi.org/wp-content/uploads/2015/ 09/PB $\left\{\backslash_{-}\right\}$From $\left\{\backslash_{-}\right\}$Filed $\left\{\backslash_{-}\right\}$to $\left\{\backslash_{-}\right\}$fork $\left\{\backslash_{-}\right\} 2008$.pdf. 
Mahajan, S., \& van Ryzin, G. (2001). Stocking retail assortments under dynamic consumer substitution. Operations Research, 49. doi: 10.1287/opre.49.3.334.11210.

McGillivray, A. R., \& Silver, E. A. (1978). Some concepts for inventory control under substituable demand. INFOR Journal, 16. doi: 10.1080/03155986.1978.11731687.

Minner, S., \& Transchel, S. (2017). Order variability in perishable product supply chains. European Journal of Operational Research, 260. doi: 10.1016/j.ejor.2016.12.016.

Mondéjar-Jiménez, J.-A., Ferrari, G., Secondi, L., \& Principato, L. (2016). From the table to waste: An exploratory study on behaviour towards food waste of Spanish and Italian youths. Journal of Cleaner Production, 138. doi: 10.1016/j.jclepro.2016.06.018.

Monier, V., Mudgal, S., Escalon, V., O'Connor, C., Gibon, T., Anderson, G., Montoux, H., Reisinger, H., Dolley, P., Ogilvie, S., \& Morton, G. (2010). Preparatory study on food waste across EU27. European Commission, Brusseles, Belgium. Retrieved from http://ec.europa.eu/environment/eussd/pdf/ bio $\left\{\backslash_{-}\right\}$foodwaste $\left\{\backslash_{-}\right\}$report.pdf. doi: $10.2779 / 85947$.

Nahmias, S. (1982). Perishable Inventory Theory: A Review. Operations Research, 30. doi: 10.1287 /opre.30.4.680.

Najafi, M., Ahmadi, A., \& Zolfagharinia, H. (2017). Blood inventory management in hospitals: Considering supply and demand uncertainty and blood transshipment possibility. Operations Research for Health Care, 15. doi: 10.1016/j.orhc.2017.08.006.

Neter, J. E., Dijkstra, S. C., Visser, M., \& Brouwer, I. A. (2016). Dutch food bank parcels do not meet nutritional guidelines for a healthy diet. British Journal of Nutrition, 116 . doi: $10.1017 /$ S0007114516002087.

Netessine, S., \& Rudi, N. (2003). Centralized and competitive inventory models with demand substitution. Operations Research, 51. doi: 10.1287/opre.51.2.329.12788.

Oishi, K., Kumagai, H., \& Hirooka, H. (2011). Application of the modified feed formulation to optimize economic and environmental criteria in beef cattle fattening systems with food by-products. Animal Feed Science and Technology, 165. doi: 10.1016/j.anifeedsci.2011.02.015.

Orgut, I. S., Ivy, J., Uzsoy, R., \& Wilson, J. R. (2015). Modeling for the equitable and effective distribution of donated good under capacity constraints. IIE Transactions, 8830. doi: 10.1080/0740817X.2015.1063792.

Pan, X. A., \& Honhon, D. (2012). Assortment planning for vertically differentiated products. Production and Operations Management, 21. doi: 10.1111/j.19375956.2011.01259.x.

Papargyropoulou, E., Lozano, R., K. Steinberger, J., Wright, N., \& Ujang, Z. B. (2014). 
The food waste hierarchy as a framework for the management of food surplus and food waste. Journal of Cleaner Production, 76. doi: 10.1016/j.jclepro.2014.04.020.

Parfitt, J., Barthel, M., \& Macnaughton, S. (2010). Food waste within food supply chains: quantification and potential for change to 2050. Philosophical Transactions of the Royal Society B: Biological Sciences, 365. doi: 10.1098/rstb.2010.0126.

Parfitt, J., Woodham, S., Swan, E., Castella, T., \& Parry, A. (2016). Quantification of food surplus, waste, and related materials in the grocery supply chain. WRAP, Banbury, United Kingdom. Retrieved from http: //www.wrap.org.uk/content/quantification-food-surplus-waste-andrelated-materials-supply-chain-report.

Qin, Y., Wang, J., \& Wei, C. (2014). Joint pricing and inventory control for fresh produce and foods with quality and physical quantity deteriorating simultaneously. International Journal of Production Economics, 152. doi: 10.1016/j.ijpe.2014.01.005.

Rabbani, M., Pourmohammad Zia, N., \& Rafiei, H. (2016). Joint optimal dynamic pricing and replenishment policies for items with simultaneous quality and physical quantity deterioration. Applied Mathematics and Computation, 287. doi: 10.1016/ j.amc.2016.04.016.

Rajaram, K., \& Tang, C. S. (2001). The impact of product substitution on retail merchandising. European Journal of Operational Research, 135. doi: 10.1016/S03772217(01)00021-2.

Robinson, S. (2004). Simulation: the practice of model development and use. Chichester, England: John Wiley \& Sons, Ltd.

Rohmer, S., Claassen, G., \& Laporte, G. (2019). A two-echelon inventory routing problem for perishable products. Computers \& Operations Research, 10\%. doi: 10.1016/j.cor.2019.03.015.

Roodhuyzen, D., Luning, P., Fogliano, V., \& Steenbekkers, L. (2017). Putting together the puzzle of consumer food waste: Towards an integral perspective. Trends in Food Science \& Technology, 68. doi: 10.1016/j.tifs.2017.07.009.

van Ryzin, G., \& Mahajan, S. (1999). On the relationship between inventory costs and variety benefits in retail assortments. Management Science, 45. doi: 10.1287/ mnsc.45.11.1496.

Sachs, A. L. (2015). Data-driven order policies with censored demand and substitution in retailing. Lecture Notes in Economics and Mathematical Systems, 680 . doi: 10.1007/ 978-3-319-13305-8_5.

Sainathan, A. (2013). Pricing and replenishment of competing perishable product variants under dynamic demand substitution. Production and Operations Management, 22. doi: 
10.1111/poms.12004.

Scherhaufer, S., Moates, G., Hartikainen, H., Waldron, K., \& Obersteiner, G. (2018). Environmental impacts of food waste in Europe. Waste Management, 7\%. doi: 10.1016/ j.wasman.2018.04.038.

Schlapp, J., \& Fleischmann, M. (2018). Technical note-Multiproduct inventory management under customer substitution and capacity restrictions. Operations Research, 66 . doi: 10.1287 /opre.2017.1690.

Scholz, K., Eriksson, M., \& Strid, I. (2015). Carbon footprint of supermarket food waste. Resources, Conservation and Recycling, 94. doi: 10.1016/j.resconrec.2014.11.016.

Seljak, B. K. (2009). Computer-based dietary menu planning. Journal of Food Composition and Analysis, 22. doi: 10.1016/j.jfca.2009.02.006.

Sen, A. (2008). The US fashion industry: A supply chain review. International Journal of Production Economics, 114. doi: 10.1016/j.ijpe.2007.05.022.

Shah, J., \& Avittathur, B. (2007). The retailer multi-item inventory problem with demand cannibalization and substitution. International Journal of Production Economics, 106. doi: $10.1016 /$ j.ijpe.2006.04.004.

Shao, J., Krishnan, H., \& Thomas McCormick, S. (2013). Distributing a product line in a decentralized supply chain. Production and Operations Management, 22. doi: 10.1111/j.1937-5956.2012.01359.x.

Shin, H., Park, S., Lee, E., \& Benton, W. (2015). A classification of the literature on the planning of substitutable products. European Journal of Operational Research, 246. doi: 10.1016/j.ejor.2015.04.013.

Smith, S. A., \& Agrawal, N. (2000). Management of multi-item retail inventory systems with demand substitution. Operations Research, 48. doi: 10.1287/opre.48.1.50.12443.

Snels, J., Klein Gebbink, S. A., van den Boogaard, K., Delahay, E., Borgijink, J., van den Heuvel, E., Houtman, J., Kleeven, N., Koenders, L., Saris, A., \& Steeghs, V. (2012). Smakelijk eten : aandacht voor eten bij het Leger des Heils. Wageningen Food \& Biobased Research, Wageningen, the Netherlands. Retrieved from http://edepot.wur.nl/211864.

Soethoudt, J. M., van der Sluis, A. A., Waarts, Y. R., \& Tromp, S. O. (2012). Houdbaarheidsdatum, verspilde moeite?. Wageningen Food \& Biobased Research, Wageningen, the Netherlands.

Solak, S., Scherrer, C., \& Ghoniem, A. (2014). The stop-and-drop problem in nonprofit food distribution networks. Annals of Operations Research, 221. doi: 10.1007/s10479012-1068-7. 
Sprake, E. F., Russell, J. M., \& Barker, M. E. (2014). Food choice and nutrient intake amongst homeless people. Journal of Human Nutrition and Dietetics, 27. doi: 10.1111/ jhn. 12130 .

Stenmarck, A., Jensen, C., Quested, T., \& Moates, G. (2016). FUSIONS Food waste data set for EU-28. European Comission, Stockholm, Sweden. Retrieved from http://www.eu-fusions.org/phocadownload/Publications/ EstimatesofEuropeanfoodwastelevels.pdf.

Stenmarck, A., Werge, M., Hanssen, O. J., Silvennoinen, K., \& Katajajuuri, J.-M. (2011). Initiatives on prevention of food waste in the retail and wholesale trades. IVL, Copenhagen, Denmark. Retrieved from http://urn.kb.se/resolve?urn=urn:nbn: se: norden: org: diva-1655.

Stigler, G. J. (1945). The cost of subsistence. Journal of Farm Economics, 27. doi: $10.2307 / 1231810$.

Tan, B., \& Karabati, S. (2013). Retail inventory management with stock-out based dynamic demand substitution. International Journal of Production Economics, 145. doi: $10.1016 /$ j.ijpe.2012.10.002.

Teller, C., Holweg, C., Reiner, G., \& Kotzab, H. (2018). Retail store operations and food waste. Journal of Cleaner Production, 185. doi: 10.1016/j.clepro.2018.02.280.

Thyberg, K. L., \& Tonjes, D. J. (2016). Drivers of food waste and their implications for sustainable policy development. Resources, Conservation and Recycling, 106. doi: 10.1016/j.resconrec.2015.11.016.

Transchel, S. (2017). Inventory management under price-based and stockout-based substitution. European Journal of Operational Research, 262. doi: 10.1016/j.ejor.2017.03.075.

Transchel, S., \& Minner, S. (2009). Dynamic pricing and replenishment in the warehouse scheduling problem-A common cycle approach. International Journal of Production Economics, 118. doi: 10.1016/j.ijpe.2008.08.046.

Tromp, S.-O., Haijema, R., Rijgersberg, H., \& van der Vorst, J. G. (2016). A systematic approach to preventing chilled-food waste at the retail outlet. International Journal of Production Economics, 182. doi: 10.1016/J.IJPE.2016.10.003.

Tromp, S. O., Rijgersberg, H., Pereira Da Silva, F., \& Bartels, P. (2012). Retail benefits of dynamic expiry dates - Simulating opportunity losses due to product loss, discount policy and out of stock. International Journal of Production Economics, 139. doi: 10.1016/j.ijpe.2011.04.029.

Tsiros, M., \& Heilman, C. M. (2005). The effect of expiration dates and perceived risk on purchasing behavior in grocery store perishable categories. Journal of Marketing, 69 . doi: 10.1509/jmkg.69.2.114.60762. 
Vaagen, H., Wallace, S. W., \& Kaut, M. (2009). Modelling consumer-directed substitution. International Journal of Production Economics, 134. doi: 10.1016/ j.ijpe.2009.11.012.

Van Westerhoven, M. (2013). Bepaling voedselverliezen in huishoudelijk afval in Nederland. CREM, Amsterdam, the Netherlands. Retrieved from https: //www.rijksoverheid.nl/documenten/rapporten/2014/01/13/bepalingvoedselverliezen-in-huishoudelijk-afval-in-nederland-vervolgmeting2013.

Van Woensel, T., Van Donselaar, K., Broekmeulen, R., \& Fransoo, J. (2007). Consumer responses to shelf out-of-stocks of perishable products. International Journal of Physical Distribution \& Logistics Management, 37. doi: 10.1108/09600030710840822.

Voedselbank-Nederland (2018). Feiten en Cijfers Voedselbanken Nederland2018. Voedselbanken Nederland, Houten, the Netherlands. Retrieved from https://voedselbankennederland.nl/wp-content/uploads/2018/08/Feitenen-Cijfers-per-31-12-2017-DEF.pdf.

Wan, M., Huang, Y., Zhao, L., Deng, T., \& Fransoo, J. (2018). Demand estimation under multi-store multi-product substitution in high density traditional retail. European Journal of Operational Research, 266. doi: 10.1016/j.ejor.2017.09.014.

Wang, X., \& Disney, S. M. (2016). The bullwhip effect: Progress, trends and directions. European Journal of Operational Research, 250. doi: 10.1016/j.ejor.2015.07.022.

Wang, X., \& Li, D. (2012). A dynamic product quality evaluation based pricing model for perishable food supply chains. Omega, 40. doi: 10.1016/j.omega.2012.02.001.

Westhoek, H., J., Ingram, J., Van Berkum, S., Özay, L., \& Hajer, M. (2016). Food systems and natural resources. UNEP, Nairobi, Kenya. Retrieved from http: //www.resourcepanel.org/reports/food-systems-and-natural-resources.

Whitehead, P., Palmer, M., Mena, C., Williams, A., \& Walsh, C. (2011). Resource maps for fresh meat across retail and wholesale supply chains. WRAP, Banbury, United Kingdom. Retrieved from https://pdxscholar.library.pdx.edu/cgi/ viewcontent.cgi?article $=1108\{\backslash \&\}$ context=busadmin $\{\backslash\}$ fac.

Wicks, R., Trevena, L. J., \& Quine, S. (2006). Experiences of food insecurity among urban soup kitchen consumers: Insights for improving nutrition and well-being. Journal of the American Dietetic Association, 106. doi: 10.1016/j.jada.2006.03.006.

Wilson, L. C., Alexander, A., \& Lumbers, M. (2004). Food access and dietary variety among older people. International Journal of Retail \& Distribution Management, 32. doi: 10.1108/09590550410521789.

Wollenburg, J., Hübner, A., Kuhn, H., \& Trautrims, A. (2018). From bricks-and-mortar 
to bricks-and-clicks. International Journal of Physical Distribution $\&$ Logistics Management, 48. doi: 10.1108/ijpdlm-10-2016-0290.

World Bank (2007). World development report 2008: Agriculture for development - overview. World Bank, Washington DC, United States. Retrieved from http://documents.worldbank.org/curated/en/873611468174885835/ World-development-report-2008-agriculture-for-development-overview.

WRAP (2019). Why take action: legal/policy case. Retrieved from http:// www.wrap.org.uk/content/why-take-action-legalpolicy-case.

Wu, D., Zhang, B., \& Baron, O. (2018). A trade credit model with asymmetric competing retailers. Production and Operations Management, 28. doi: 10.1111/poms.12882.

Yücel, E., Karaesmen, F., Salman, F. S., \& Türkay, M. (2011). Optimizing product assortment under customer-driven demand substitution. European Journal of Operational Research, 199. doi: 10.1016/j.ejor.2008.08.004.

Zeppetella, L., Gebennini, E., Grassi, A., \& Rimini, B. (2017). Optimal production scheduling with customer-driven demand substitution. International Journal of Production Research, 55. doi: 10.1080/00207543.2016.1223895.

Zhang, J., Wang, Y., Lu, L., \& Tang, W. (2015). Optimal dynamic pricing and replenishment cycle for non-instantaneous deterioration items with inventory-leveldependent demand. International Journal of Production Economics, 170 . doi: 10.1016/ j.ijpe.2015.09.016.

Zhao, W., \& Zheng, Y.-S. (2000). Optimal dynamic pricing for perishable assets with nonhomogeneous demand. Management Science, 46. doi: 10.1287/mnsc.46.3.375.12063. 


\section{Summary}

In this thesis, the focus is on the reduction of food waste at the retailer level, either by reducing the food waste that is generated, or by re-using food leftovers. The problem is studied with an Operations Research approach, i.e. by the development of simulation and optimization models. The reduction of food waste is important, as the environmental impact of the food system is large, e.g. $24 \%$ of the greenhouse gasses in Europe are related to the food system (Westhoek et al., 2016). As global food loss is estimated at $50 \%$ of the total production, the environmental impact of the food waste is substantial. Furthermore, the economic impact of waste is estimated on $€ 143$ million for the European countries only (Stenmarck et al., 2016). This thesis considers avoidable food waste, and thus does not include food losses or unavoidable food waste.

The focus of this thesis is on food waste at retail level. Although waste levels by the retailer are only $5 \%$, there are several reasons why it is important to address the retailers' food waste. The retailers fulfil a key position in many food supply chains, as they sell many products from different food supply chains. Moreover, they are the last actor in the supply chain where logistic interventions can be implemented. Three main causes for avoidable food waste are identified, (i) the consumer in-store behaviour and the consumer demand; (ii) the store management e.g. the replenishment policies and (iii) the product shelf life. In the chapters of this thesis, several interventions are considered in order to reduce or re-use food waste by the retailer.

In Chapter 2, both the replenishment strategy and the consumer in-store behaviour are addressed to obtain a food waste reduction. A discount on nearly expired products is provided, to stimulate consumers to buy these products, instead of the fresher ones, which could be sold at a later point in time. Furthermore, a shelf life based on the actual quality of the product (dynamic shelf life) is applied instead of a fixed, printed date. A simulation-based optimization model is created to optimize the replenishment quantity of the retailer combined with the two interventions to reduce food waste. Depending on the discount given, waste reductions up to $60 \%$ can be achieved when a discount is applied, although profit levels will be reduced with $11 \%$. A dynamic shelf life can give a waste reduction around $40 \%$ on average, without a loss of profit. 
The replenishment decision for substitutable products is considered in Chapter 3, addressing the in-store behaviour of consumers. The replenishment of perishable products is optimized together, i.e. the replenishment decisions of one product depends on the replenishment decision of the other product. Moreover, the substitution behaviour of consumers between these products is included. For a two-product case, with one-way substitution, a simulation-based optimization model is presented to determine the optimal order-up-to levels. By combining the replenishment decisions for the two products, an average waste reduction of $35 \%$ is obtained. Results show high waste reductions when the willingness to substitute of consumers is high. Moreover, large improvements can be made when the two products differ in shelf life or profit margin.

Chapter 4 also includes substitution behaviour in the inventory decisions, but for vertically differentiated products with two-way substitution. A heuristic is presented to optimize the replenishment decisions for multiple products under multiple substitution rounds. The results show the importance of including the inventory levels when deciding on the assortment to carry in the store. Furthermore, the incorporation of multiple substitution rounds has a significant effect on the profit. Moreover, due to demand uncertainty, the inclusion of product substitution and thus combining the inventory decisions for a product group increases the expected profit. The demand uncertainty can be shared among the products within the product group, which reduces the relative uncertainty, and profit levels therefore might rise.

At the retailer, it will be impossible to reduce the food waste to zero. The re-use of food leftovers is therefore studied in Chapter 5. A mixed integer linear programming (MILP) model is developed for the menu planning of a soup kitchen. The soup kitchen can buy ingredients, but also receives food donations from retailers or other food companies. By minimizing costs, the MILP model provides a menu plan for the soup kitchen with restrictions on meal and ingredient use to provide a varied and healthy meal to the customers of the soup kitchen. Donations either are provided via a contract between the retailer and the soup kitchen, or arrive on a random basis. Results show the cost reduction for the soup kitchen. Furthermore, when good agreements are made between the retailer and the soup kitchen, 50-70\% of the food waste obtained at the retailer can be re-used.

In Chapter 6 integrated conclusions are presented combined with a discussion and an outlook to further research. All interventions show a great possibility to reduce food waste, and several linkages between the interventions can be found. By combining the interventions, food waste at the retailer can be addressed at several points, which will increase the total effect of the interventions mentioned before. 


\section{Publications}

\section{In this dissertation}

Buisman, M. E., Haijema, R., \& Bloemhof-Ruwaard, J. M. (2019). Discounting and dynamic shelf life to reduce fresh food waste at retailers. International Journal of Production Economics, 209. doi: 10.1016/j.ijpe.2017.07.016

Buisman, M. E., Haijema, R., Akkerman, R., \& Bloemhof, J. M. (2019). Donation management for menu planning at soup kitchens. European Journal of Operational Research, 272. doi: 10.1016/j.ejor.2018.06.005

Buisman, M. E., Haijema, R., \& Hendrix, E. M. T. (Submitted). Product substitution to increase profit and reduce food waste at retailers.

Buisman, M. E., Transchel, S., \& Haijema, R. (In preparation). Inventory optimization under consumer driven substitution for vertically differentiated products

\section{Other scientific publications}

Buisman, M. E., Haijema, R., \& Hendrix, E. M. T. (2018). On the $\delta$-service level for demand substitution in inventory control. IFAC-PapersOnLine, 51. doi: 10.1016/j.ifacol.2018.08.483

Buisman, M. E., \& Jonkman, J. (2019). Dietary trends from 1950 to 2010: a Dutch cookbook analysis. Journal of Nutritional Science, 8. doi: 10.1017/jns.2019.3 
Hendrix, E. M. T., Ortega, G., Haijema, R., Buisman, M. E., \& García, I. (2019). On computing optimal policies in perishable inventory control using value iteration. Computational and Mathematical Methods, 1. doi: 10.1002/cmm4.1027 


\section{Acknowledgements}

Although many people have different opinions, I honestly regret to finish my PhD. Not because I should not have started it in the first place, but because I don't want it to be finished yet. I really hope that after my $\mathrm{PhD}$ research will not only become a hobby but that remains a job.

For now, I want to thank several persons. Of course, I want to thank my two supervisors Rene and Jacqueline. Without you I would not have gotten to this point. During the last four years, I always felt supported in anything I did or wanted to do. I can't remember any of my ideas or activity wishes being denied. On the contrary, the amount of conferences or trips they suggested was more than I could handle. (Note to everyone who thought I was never at home: I could really have travelled so much more than I did during my $\mathrm{PhD}$ ). I am very thankful for all the possibilities you both created to develop myself, I gained much more knowledge on OR topics, got teaching and supervision skills and I got to know myself better.

Jacqueline, at the start of my $\mathrm{PhD}$, your ideas and suggestions were always most welcome. Even the suggestions to talk more about the process, and not the project in our regular meetings. Later, when you got your own struggles, you were still there whenever I needed. Rene, thank you for all your support, thank you for pushing me into topics I might not wanted and for believing in my capability of understanding these topics, even though I did not believe in myself. Although I did not always like your suggestions at first, they mostly turned out to be a lot of fun and got my research to a higher level.

Sandra, Renzo and Eligius, I have enjoyed the collaboration with all of you. Being able to work together does, in my opinion, improve research and makes it much more fun to do!

This PhD thesis is part of the EU Horizon-2020 project REFRESH. I am grateful for the financial support I received and the discussions with the other project partners. These discussions were valuable to me and helped to see my work in a broader perspective.

Sometimes the biggest impact is the one that is not obviously visible. There are many people who contribute to this thesis, although this is not visible on paper. I'm very happy 
to have had so many nice colleagues over the last years, both at ORL and INF. Thank you all for your support and advice. Also, many thanks for the distractions to keep me off work, without this I would have been finished even earlier, and no one wanted that. A special thanks to Sonja. It was great to share an office with you all these years. Without our office-karaoke, cheese fondue and endless discussions on any topic we could think of, it would have been a lot less fun.

Nynke, Dineke and Jochem, over the last years your places feel like home to me. Whenever I was completely stressed out I could always sit on your couch and relax. Of course, I am also very happy with all the less stressful moments we spend together. I have met many more great people in my life. Even though it is too much to mention everyone by name, a word of thanks is also owed to you!

Papa, mama, Johanneke and Thomas, a special thanks to you as well. I hope you are as proud as I am at this moment. Even though you not always knew what I did work on, or why I was again somewhere outside the Netherlands, I felt your support. I love you all.

Finally, Matthias, thank you for being there, even when you weren't there. I'm very grateful for your ability to let me carry out most of my plans, even though that resulted in my being even further away from you. I'm curious what the future will bring us.

Marjolein 


\section{Completed Training and Supervision Plan}

Marjolein Elize Buisman

Wageningen School of Social Sciences (WASS)

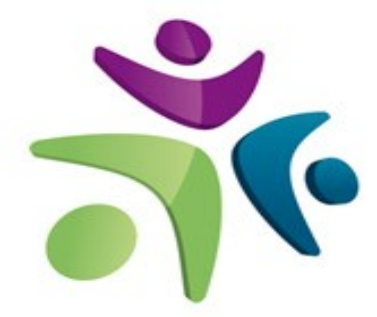

Wageningen School of Social Sciences

\begin{tabular}{llcc}
\hline Name of the learning activity & Department/Institute & Year & ECTS* \\
\hline A) Project related competences & & & \\
\hline Writing a research proposal & WUR & 2016 & 6 \\
Programming in Phyton, INF-22306 & WUR & 2015 & 6 \\
Quantitative modelling and analysis & GP-OML & 2016 & 1 \\
of supply chains & LNMB & 2016 & 1 \\
Stochastic Programming & GP-OML & 2016 & 1 \\
Advanced inventory theory & WUR & 2016 & 6 \\
Predicting food quality, FQD-31306 & GP-OML & 2017 & 4 \\
\hline Markov Decision Process & & &
\end{tabular}

Continued on next page 


\begin{tabular}{|c|c|c|c|}
\hline Name of the learning activity & Department/Institute & Year & ECTS* \\
\hline \multicolumn{4}{|c|}{ B) General research related competences } \\
\hline Introduction course & WASS & 2016 & 1 \\
\hline Competence assessment & WGS & 2016 & 0.3 \\
\hline Interpersonal communication & WGS & 2016 & 0.6 \\
\hline Data management planning & Library WUR & 2016 & 0.4 \\
\hline Reviewing a scientific paper & WGS & 2017 & 0.1 \\
\hline $\begin{array}{l}\text { Reviewing scientific papers for: } \\
\text { - International Journal of pro- } \\
\text { duction economics } \\
\text { - European Journal of Opera- } \\
\text { tions Research } \\
\text { - Computers in Industry }\end{array}$ & Elsevier & $2016-2019$ & 3 \\
\hline $\begin{array}{l}\text { 'Dynamic pricing and dynamic shelf } \\
\text { life to reduce (perishable) food waste } \\
\text { at retailers' }\end{array}$ & $\begin{array}{l}\text { 19th International symposium } \\
\text { on inventory research }\end{array}$ & 2016 & 1 \\
\hline $\begin{array}{l}\text { 'Donation management at soup } \\
\text { kitchens' }\end{array}$ & WASS PhD day & 2017 & 1 \\
\hline $\begin{array}{l}\text { 'Product substitution to reduce food } \\
\text { waste and increase profit' }\end{array}$ & $\begin{array}{l}3^{r d} \text { workshop of the EURO } \\
\text { working group on retail oper- } \\
\text { ations }\end{array}$ & 2018 & 1 \\
\hline $\begin{array}{l}\text { 'Donation management of menu } \\
\text { planning at soup kitchens' }\end{array}$ & $\begin{array}{l}29^{\text {th }} \text { European conference on } \\
\text { Operations Research }\end{array}$ & 2018 & 1 \\
\hline $\begin{array}{l}\text { 'Inventory optimization for vertically } \\
\text { differentiated products under con- } \\
\text { sumer driven substitution' }\end{array}$ & $\begin{array}{l}4^{\text {th }} \text { workshop of the EURO } \\
\text { working group on retail oper- } \\
\text { ations }\end{array}$ & 2019 & 1 \\
\hline
\end{tabular}

Supervision of BSc and MSc thesis students on topics related to inventory management and/or simulation and optimization of (part of) the WUR 2016-2019 2 food supply chain Teaching/Teaching assistance in Operations Research and Logistics courses

Masterclass 'De sucessvolle introvert'

WUR

2016-2019 2

Project meetings

2016 1.4

Lecturing

REFRESH

2015-2019

2

Start to teach

ESC

2018

2018

ESC 1

Total

*One credit according to ECTS is on average equivalent to 28 hours of study load 

This thesis is part of the $\mathrm{PhD}$ thesis series of the Beta Research School for Operations Management and Logistics (onderzoeksschool-beta.nl) in which the following universities cooperate: Eindhoven University of Technology, Maastricht University, University of Twente, VU Amsterdam, Wageningen University and Research, KU Leuven and Universiteit Hasselt.

The studies presented in this thesis were performed within the REFRESH project, as part of the Horizon 2020 Framework Programme of the European Union under Grant Agreement no. 641933. The content of this thesis is the sole responsibility of REFRESH and can in no way be taken to reflect the views of the European Union.

Cover design by Dennis Hendriks - ProefschriftMaken.nl 
